\title{
DIMENSIONS OF RANDOM COVERING SETS IN RIEMANN MANIFOLDS
}

\author{
BY DE-JUN FENG*, ${ }^{*}$, ESA JÄRVENPÄ̈̈ ${ }^{\dagger, 2}$, MAARIT JÄRVENPÄÄ ${ }^{\dagger, 2}$ AND \\ VILLE SUOMALA ${ }^{\dagger, 2}$
}

Chinese University of Hong Kong* and University of Oulu ${ }^{\dagger}$

Let $\mathbf{M}, \mathbf{N}$ and $\mathbf{K}$ be $d$-dimensional Riemann manifolds. Assume that $\mathbf{A}:=$ $\left(A_{n}\right)_{n \in \mathbb{N}}$ is a sequence of Lebesgue measurable subsets of $\mathbf{M}$ satisfying a necessary density condition and $\mathbf{x}:=\left(x_{n}\right)_{n \in \mathbb{N}}$ is a sequence of independent random variables, which are distributed on $\mathbf{K}$ according to a measure, which is not purely singular with respect to the Riemann volume. We give a formula for the almost sure value of the Hausdorff dimension of random covering sets $\mathbf{E}(\mathbf{x}, \mathbf{A}):=\lim \sup _{n \rightarrow \infty} A_{n}\left(x_{n}\right) \subset \mathbf{N}$. Here, $A_{n}\left(x_{n}\right)$ is a diffeomorphic image of $A_{n}$ depending on $x_{n}$. We also verify that the packing dimensions of $\mathbf{E}(\mathbf{x}, \mathbf{A})$ equal $d$ almost surely.

\section{CONTENTS}

1. Introduction and main theorem . . . . . . . . . . . . . . . . . . . . . . . . . . 1542

2. Auxiliary results . . . . . . . . . . . . . . . . . . . . . . . 1550

3. Upper bound for Hausdorff dimension . . . . . . . . . . . . . . . . . . 1555

4. Minimal regular energy . . . . . . . . . . . . . . . . . . . . . . 1566

5. Lower bound for Hausdorff dimension . . . . . . . . . . . . . . . . . . . . . . 1572

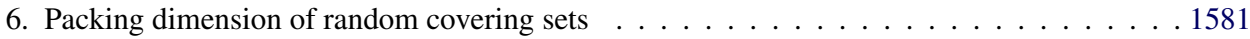

7. Proof of Corollary 1.2 and examples . . . . . . . . . . . . . . . . . . . 1587

8. Further generalisations and remarks . . . . . . . . . . . . . . . . . . . 1590

8.1. A weak large intersection property of random covering sets . . . . . . . . . . . 1590

8.2. Hausdorff measure of random covering sets . . . . . . . . . . . . . . . . . 1591

8.3. A question on the measurability of level sets of random covering sets . . . . . . . 1593

Acknowledgements . . . . . . . . . . . . . . . . . . . . . . 1593

References . . . . . . . . . . . . . . . . . . . . . . . . . 1593

1. Introduction and main theorem. Limsup sets, defined as upper limits of various sequences of sets, play an important role in different areas of mathematics. One of their earliest occurrences originates from the study of random placement

Received February 2016; revised February 2017.

${ }^{1}$ Supported by the RGC grants in the Hong Kong Special Administrative Region, China (projects CUHK401112, CUHK401013).

${ }^{2}$ Supported by the Centre of Excellence in Analysis and Dynamics Research funded by the Academy of Finland.

MSC2010 subject classifications. 60D05, 28A80.

Key words and phrases. Random covering set, Hausdorff dimension, packing dimension. 
of circular arcs in the unit circle by Borel [7] in the late 1890s. He stated that a given point belongs to infinitely many arcs provided that the placement of arcs is random and the sum of their lengths is infinite. This statement is the origin of what is nowadays known as the Borel-Cantelli lemma. We refer to [37] for more details and references on the historical development. Related to geometric measure theory and fractals, limsup sets appear implicitly already in the investigation of the Besicovitch-Eggleston sets concerning the $k$-adic expansions of real numbers [5, 16]. They play also a central role in Diophantine approximation. For instance, the classical theorems of Khintchine and Jarnik provide size estimates in terms of Lebesgue and Hausdorff measure for limsup sets consisting of well-approximable numbers (cf. [26]).

In the modern language, random covering sets are a class of limsup sets defined by means of a family of randomly distributed subsets of the $d$-dimensional torus $\mathbb{T}^{d}:=\mathbb{R}^{d} / \mathbb{Z}^{d}$. Supposing that $\mathbf{A}:=\left(A_{n}\right)_{n \in \mathbb{N}}$ is a deterministic sequence of nonempty subsets of $\mathbb{T}^{d}$ and $\mathbf{x}:=\left(x_{n}\right)_{n \in \mathbb{N}}$ is a sequence of independent random variables which are uniformly distributed on $\mathbb{T}^{d}$, define a random covering set $\mathbf{E}(\mathbf{x}, \mathbf{A})$ by

$$
\mathbf{E}(\mathbf{x}, \mathbf{A}):=\limsup _{n \rightarrow \infty}\left(x_{n}+A_{n}\right)=\bigcap_{n=1}^{\infty} \bigcup_{k=n}^{\infty}\left(x_{k}+A_{k}\right),
$$

where $x+A:=\{x+y: y \in A\}$. We denote the Lebesgue measure on $\mathbb{T}^{d}$ by $\mathcal{L}$. It is easy to see that $\mathcal{L}(\mathbf{E}(\mathbf{x}, \mathbf{A}))=0$ for all $\mathbf{x}$ if the series $\sum_{k=1}^{\infty} \mathcal{L}\left(A_{k}\right)$ converges. On the other hand, it follows from the Borel-Cantelli lemma and Fubini's theorem that $\mathcal{L}(\mathbf{E}(\mathbf{x}, \mathbf{A}))=1$ almost surely provided the sets $A_{n}$ are Lebesgue measurable and the series $\sum_{k=1}^{\infty} \mathcal{L}\left(A_{k}\right)$ diverges. Note that this result is essentially the higher dimensional analogue of Borel's original idea concerning the covering of the circle by random arcs, which we discussed in the beginning of this section.

The case of full Lebesgue measure has been extensively studied. In 1956, Dvoretzky [15] posed a problem of finding conditions which guarantee that the whole torus $\mathbb{T}^{d}$ is covered almost surely. Even in the simplest case when $d=1$ and the generating sets are intervals of length $\left(l_{n}\right)_{n \in \mathbb{N}}$, this problem, known in literature as the Dvoretzky covering problem, turned out to be rather long-standing. After substantial contributions of many authors, including Billard [6], Erdős [19], Kahane [34] and Mandelbrot [44], the full answer was given in this context by Shepp [50] in 1972. He verified that $\mathbf{E}(\mathbf{x}, \mathbf{A})=\mathbb{T}^{1}$ almost surely if and only if

$$
\sum_{n=1}^{\infty} \frac{1}{n^{2}} \exp \left(l_{1}+\cdots+l_{n}\right)=\infty
$$

where the lengths $\left(l_{n}\right)_{n \in \mathbb{N}}$ are in decreasing order. In full generality, the Dvoretzky covering problem is still unsolved. The higher dimensional case has been studied by El Hélou [18] and Kahane [36] among others. In [36], a complete solution is provided in the case when generating sets are similar simplexes. 
For various other aspects of random covering sets, we refer to [1, 18, 22, 23, 27, $29,30,35-37,41,43,51]$. Recent contributions to the topic include various types of dynamical models (see [24, 33, 42]) and projectional properties [11].

Further motivation to study limsup sets stems from Diophantine approximation. Recall that, for $\phi: \mathbb{N} \rightarrow] 0, \infty[$, the set of $\phi$ well-approximable numbers consists of those $x \in \mathbb{R}$ for which there exist infinitely many $q \in \mathbb{N}$ such that

$$
\left|x-\frac{p}{q}\right|<\phi(q)
$$

for some $p \in \mathbb{Z}$. Given $\phi$, the determination of the size of these limsup sets and various variants is an important theme in Diophantine approximation and there is a vastly growing literature on this branch of metric number theory; see [2,3] and the references therein.

In the circle $\mathbb{T}^{1}$, the study of $\phi$ well-approximable numbers may be regarded as a variant of the shrinking target problem or dynamical Diophantine approximation formulated in the following manner: assuming that $X$ is a metric space, $T: X \rightarrow X$ is a dynamical system, $\left(r_{n}\right)_{n \in \mathbb{N}}$ is a sequence of positive real numbers and $x_{0} \in X$, determine the size of the set

$$
\limsup _{n \rightarrow \infty} T^{-n}\left(B\left(x_{0}, r_{n}\right)\right)=\left\{x \in X: T^{n}(x) \in B\left(x_{0}, r_{n}\right) \text { for infinitely many } n \in \mathbb{N}\right\},
$$

where $B(x, r)$ is the open ball with radius $r$ centred at $x \in X$. Indeed, letting $x_{0}=$ $0, r_{q}=q \phi(q)$ and $T_{x}: \mathbb{T}^{1} \rightarrow \mathbb{T}^{1}$ be the rotation by an angle $x$, we recover that the set of $\phi$ well-approximable numbers consists of those $x$ such that $T_{x}^{q}(0) \in B\left(0, r_{q}\right)$ for infinitely many $q \in \mathbb{N}$. Another variant of this question, called the moving target problem, is concerned with the investigation of the limsup set

$$
\left\{x \in X: x \in B\left(T^{n}\left(x_{0}\right), r_{n}\right) \text { for infinitely many } n \in \mathbb{N}\right\} ;
$$

see $[4,8]$. A recent account on this line of research is provided in [24]. Observe that, by replacing the map $T$ with the random walk on $\mathbb{T}^{d}$ driven by the Lebesgue measure, the random covering set $\mathbf{E}(\mathbf{x}, \mathbf{A})$ may be viewed as a moving target problem limsup set provided $A_{n}=B\left(0, r_{n}\right)$ for all $n \in \mathbb{N}$. For an interesting application of limsup sets to the study of Brownian motion, we refer to [39].

In this paper, we focus on the natural problem of determining almost sure values of Hausdorff and packing dimensions of random covering sets in the case when they have zero Lebesgue measure. We denote the Hausdorff and packing dimensions by $\operatorname{dim}_{\mathrm{H}}$ and $\operatorname{dim}_{\mathrm{P}}$, respectively. For $d=1$ and for an arbitrary decreasing sequence $\mathbf{A}=\left(A_{n}\right)_{n \in \mathbb{N}}$ of intervals of lengths $\left(l_{n}\right)_{n \in \mathbb{N}}$, the almost sure Hausdorff dimension of the random covering set is given by

$$
\operatorname{dim}_{\mathrm{H}} \mathbf{E}(\mathbf{x}, \mathbf{A})=\inf \left\{0<t \leq 1: \sum_{n=1}^{\infty}\left(l_{n}\right)^{t}<\infty\right\}=\limsup _{n \rightarrow \infty} \frac{\log n}{-\log l_{n}} .
$$


For $l_{n}=n^{-\alpha}, \alpha>1$, this is proved by Fan and $\mathrm{Wu}$ [25] and, as explained in their paper, the method works also for more general decreasing sequences $\left(l_{n}\right)_{n \in \mathbb{N}}$. Using an approach different from that of [25], Durand [14] generalised the result of [25] and obtained a dichotomy result for the Hausdorff measure of $\mathbf{E}(\mathbf{x}, \mathbf{A})$ for general gauge functions. The dimension result (1.1), as well as its analogy in $\mathbb{T}^{d}$ for random coverings with balls, can also be derived from the mass transference principle proved by Beresnevich and Velani in [3] (see [31] for details). In addition to random covering sets, the mass transference technique has proved to be a useful tool in studying the limsup sets in the context of Diophantine approximation and shrinking target problems; see, for example, [2, 3, 24, 28]. However, its applicability is essentially limited to the case when the sequence A consists of balls and, therefore, it cannot be utilised in the general setting of this paper.

Notice that the methods used in $[14,25]$ rely essentially on the ambient space being a torus and generating sets being balls. One needs to employ new ideas in investigating random covering sets generated by more general sets. The case when the generating sets are rectangle-like was first studied in [31]. More precisely, assume that the generating sets in $\mathbf{A}$ are of the form $A_{n}=\Pi\left(L_{n}(R)\right)$ for all $n \in \mathbb{N}$, where $\Pi: \mathbb{R}^{d} \rightarrow \mathbb{T}^{d}$ is a natural covering map, $R$ is a subset of the closed unit cube $[0,1]^{d}$ with nonempty interior and, for all $n \in \mathbb{N}$, the map $L_{n}: \mathbb{R}^{d} \rightarrow \mathbb{R}^{d}$ is a contracting linear injection such that the sequences of singular values of $\left(L_{n}\right)_{n \in \mathbb{N}}$ decrease to 0 as $n$ tends to infinity. Note that the singular values of $L_{n}$ are the lengths of the semi-axes of $L_{n}(B(0,1))$. Under this assumption, according to [31], almost surely the Hausdorff dimension of $\mathbf{E}(\mathbf{x}, \mathbf{A})$ is given in terms of singular value functions $\Phi^{t}\left(L_{n}\right)$ (for the definition see [31]), that is, almost surely

$$
\operatorname{dim}_{\mathrm{H}} \mathbf{E}(\mathbf{x}, \mathbf{A})=\inf \left\{0<t \leq d: \sum_{n=1}^{\infty} \Phi^{t}\left(L_{n}\right)<\infty\right\}
$$

with the interpretation $\inf \varnothing=d$.

In [47], Persson proved that (1.2) remains valid when dropping off the monotonicity assumption on the singular values of $\left(L_{n}\right)_{n \in \mathbb{N}}$ in [31]. Indeed, he showed that, for a sequence $\mathbf{A}$ of open subsets of $\mathbb{T}^{d}$, almost surely

$$
\operatorname{dim}_{\mathrm{H}} \mathbf{E}(\mathbf{x}, \mathbf{A}) \geq \inf \left\{0<t \leq d: \sum_{n=1}^{\infty} g_{t}\left(A_{n}\right)<\infty\right\},
$$

where

$$
g_{t}(F):=\frac{\mathcal{L}(F)^{2}}{I_{t}(F)}
$$

for all Lebesgue measurable sets $F \subset \mathbb{T}^{d}$ with $\mathcal{L}(F)>0$, and

$$
I_{t}(F):=\iint_{F \times F}|x-y|^{-t} d \mathcal{L}(x) d \mathcal{L}(y)
$$


is the $t$-energy of $F$. For simplicity, we use the notation $|x-y|$ for both the Euclidean distance and the natural distance in $\mathbb{T}^{d}$. When the generating sets of $\mathbf{A}$ are open rectangles, the lower bound in (1.3) equals the right-hand side of (1.2). Hence, the monotonicity assumption on $\left(L_{n}\right)_{n \in \mathbb{N}}$ is not needed.

Inspired by the results of $[31,47]$, we aim at an exact dimension formula for the random covering sets constructed from an arbitrary sequence $\mathbf{A}$ of open sets or, more generally, Lebesgue measurable sets satisfying a mild density condition. To this end, we introduce the following notation. For $0 \leq t<\infty$, the $t$-dimensional Hausdorff content of a set $F \subset \mathbb{R}^{d}$ is denoted by

$$
\mathcal{H}_{\infty}^{t}(F):=\inf \left\{\sum_{n=1}^{\infty}\left(\operatorname{diam} F_{n}\right)^{t}: F \subset \bigcup_{n=1}^{\infty} F_{n}\right\},
$$

where diam is the diameter of a subset of $\mathbb{R}^{d}$. For a sequence $\mathbf{A}=\left(A_{n}\right)_{n \in \mathbb{N}}$ of subsets of $\mathbb{R}^{d}$, we define

$$
t_{0}(\mathbf{A}):=\inf \left\{0<t \leq d: \sum_{n=1}^{\infty} \mathcal{H}_{\infty}^{t}\left(A_{n}\right)<\infty\right\}
$$

with the interpretation $\inf \varnothing=d$. If $\mathbf{A}$ is a sequence of Lebesgue measurable subsets of $\mathbb{R}^{d}$, we set

$$
s_{0}(\mathbf{A}):=\inf \left\{0<s \leq d: \sum_{n=1}^{\infty} G_{s}\left(A_{n}\right)<\infty\right\}
$$

with the interpretation $\inf \varnothing=d$, where

(1.9) $G_{S}(E):=\sup \left\{g_{s}(F): F \subset E, F\right.$ is Lebesgue measurable and $\left.\mathcal{L}(F)>0\right\}$

with the interpretation $\sup \varnothing=0$. Finally, given $F \subset \mathbb{R}^{d}$, we say that a point $x \in F$ has positive Lebesgue density with respect to $F$ if

$$
\liminf _{r \rightarrow 0} \frac{\mathcal{L}(F \cap B(x, r))}{\mathcal{L}(B(x, r))}>0
$$

and, moreover, the set $F$ has positive Lebesgue density if all of its points have positive Lebesgue density with respect to $F$.

As a consequence of our main theorem (see Theorem 1.1), we will prove that almost surely

$$
\operatorname{dim}_{H} \mathbf{E}(\mathbf{x}, \mathbf{A})=s_{0}(\mathbf{A})=t_{0}(\mathbf{A})
$$

provided that $\mathbf{A}=\left(A_{n}\right)_{n \in \mathbb{N}}$ is a sequence of Lebesgue measurable subsets of $\mathbb{T}^{d}$ having positive Lebesgue density. It is worth noting that $s_{0}(\mathbf{A})$ could be strictly larger than Persson's lower bound [i.e., the right-hand side of (1.3)] even when $\mathbf{A}$ consists of open sets (see Example 7.1).

Let us first give some remarks and briefly illustrate our main strategy in the proof of (1.10). The whole proof consists of three parts: $\operatorname{dim}_{H} \mathbf{E}(\mathbf{x}, \mathbf{A}) \leq t_{0}(\mathbf{A})$, 
$s_{0}(\mathbf{A})=t_{0}(\mathbf{A})$ and $\operatorname{dim}_{\mathrm{H}} \mathbf{E}(\mathbf{x}, \mathbf{A}) \geq s_{0}(\mathbf{A})$ almost surely. The assumption of positive Lebesgue density is only used in the proof of the equality $s_{0}(\mathbf{A})=t_{0}(\mathbf{A})$. Without this assumption, the equality may fail and, furthermore, it may happen that almost surely $\operatorname{dim}_{\mathrm{H}} \mathbf{E}(\mathbf{x}, \mathbf{A})<t_{0}(\mathbf{A})$ and $\operatorname{dim}_{\mathrm{H}} \mathbf{E}(\mathbf{x}, \mathbf{A})>s_{0}(\mathbf{A})$ (see Examples 7.2 and 7.4).

The proof of the upper bound [i.e., $\operatorname{dim}_{\mathrm{H}} \mathbf{E}(\mathbf{x}, \mathbf{A}) \leq t_{0}(\mathbf{A})$ ] is direct and simple. To prove the equality $s_{0}(\mathbf{A})=t_{0}(\mathbf{A})$, we manage to establish certain relations between the quantities $\mathcal{H}_{\infty}^{t}(\cdot)$ and $G_{t}(\cdot)$ (see Lemmas 3.2 and 3.10). The proof of these relations employs some potential theoretic arguments, and is rather long. A key ingredient is a subtle and technical result (Proposition 3.8), which allows us to approximate a given measure $\mu$ and its $s$-energy simultaneously by a certain sequence of normalised Lebesgue measures. As for the lower bound, we note that if $U$ is open, then a straightforward approximation argument implies that

$$
G_{S}(U)=\sup \left\{g_{s}(V): V \subset U, V \text { is open and } \mathcal{L}(V)>0\right\} .
$$

With Persson's result, this characterisation can be employed to give a more direct proof of the fact that $s_{0}(\mathbf{A})$ is a lower bound for $\operatorname{dim}_{H} \mathbf{E}(\mathbf{x}, \mathbf{A})$ in the case when $\mathbf{A}$ is a sequence of open sets. However, this method does not work if the sets in the sequence $\mathbf{A}$ fail to be open. ${ }^{3}$ For this reason, we need to make use of a completely different approach to deal with a more general generating sequence $\mathbf{A}$. For that purpose, we introduce the notion of minimal regular energy, which allows us to give a lower bound of the Hausdorff dimension of random covering sets under certain energy condition (see Section 4). A rather sophisticated random mass distribution argument is then carried out in Section 5 to verify this condition.

Regarding the packing dimension of random covering sets, we show that if the sets in $\mathbf{A}$ are Lebesgue measurable and $\mathcal{L}\left(A_{n}\right)>0$ for infinitely many $n \in \mathbb{N}$, then almost surely

$$
\operatorname{dim}_{P} \mathbf{E}(\mathbf{x}, \mathbf{A})=d
$$

For open generating sets, this result is immediate since $\mathbf{E}(\mathbf{x}, \mathbf{A})$ is a $G_{\delta}$-set, which is almost surely dense. As in the case of Hausdorff dimension, replacing open generating sets by Lebesgue measurable ones (of positive measure) turns out to be a subtle task. The strategy in the proof of (1.11) is somewhat analogous to that of (1.10). However, instead of the minimal regular energy and a mass distribution argument, we apply a result that allows us to conclude that $\operatorname{dim}_{P} \mathbf{E}(\mathbf{x}, \mathbf{A})=d$ by estimating, for compact sets $F$, the number of dyadic cubes intersecting $F \cap \bigcup_{i=n}^{\infty}\left(x_{n}+A_{n}\right)$ in a set of positive Lebesgue measure (see Proposition 6.4). Observe that since $\mathbf{E}(\mathbf{x}, \mathbf{A})$ is almost surely dense, the box counting dimension of $\mathbf{E}(\mathbf{x}, \mathbf{A})$ exists and is equal to $d$ almost surely.

\footnotetext{
${ }^{3}$ When A consists of open sets, it is also unclear whether Persson's method could be used to prove this lower bound in our more general setting, where the translations $\mathbf{x}=\left(x_{n}\right)_{n \in \mathbb{N}}$ are independent with a law not singular with respect to $\mathcal{L}$.
} 
To summarise, the equation (1.10) gives a characterisation of the almost sure value of the Hausdorff dimension of random covering sets in $\mathbb{T}^{d}$ for rather general generating sequences $\mathbf{A}$ when the translations $\mathbf{x}=\left(x_{n}\right)_{n \in \mathbb{N}}$ are independent and uniformly distributed. As illustrated by Example 7.2 (see also Example 7.4), the assumption on positive Lebesgue density cannot be replaced by the weaker assumption that $\mathcal{L}\left(A_{n} \cap B(x, r)\right)>0$ for all $r>0, x \in A_{n}$ and $n \in \mathbb{N}$. In our main result, Theorem 1.1, we will further generalise (1.10) and (1.11) in several different directions. First, we will replace the uniform distribution by an arbitrary Radon probability measure which is not purely singular with respect to the Lebesgue measure. Second, we will be able to replace the torus $\mathbb{T}^{d}$ by any open subset of $\mathbb{R}^{d}$, in particular, by $\mathbb{R}^{d}$ itself. These generalisations allow us to deduce (1.10) and (1.11) for many natural unbounded models, including the case when $\left(x_{n}\right)_{n \in \mathbb{N}}$ are independent Gaussian random variables on $\mathbb{R}^{d}$ and $\left(A_{n}\right)_{n \in \mathbb{N}}$ are Lebesgue measurable subsets with positive Lebesgue density supported on a fixed compact subset of $\mathbb{R}^{d}$. Finally, we extend (1.10) and (1.11) to Lie groups and, more generally, to smooth Riemann manifolds. To achieve this, note that when the ambient space is $\mathbb{T}^{d}$, the structure is linear in the sense that the random covering set is of the form

$$
\mathbf{E}(\mathbf{x}, \mathbf{A})=\limsup _{n \rightarrow \infty} f\left(x_{n}, A_{n}\right),
$$

where the function $f: \mathbb{T}^{d} \times \mathbb{T}^{d} \rightarrow \mathbb{T}^{d}$ is defined as $f(x, y)=x+y$. Thus, a natural attempt to extend (1.10) and (1.11) to Lie groups or, more generally, to smooth manifolds is to study a nonlinear structure where $f$ is a smooth mapping.

Before presenting our main result in full generality, we will set up some further notation. Let $U, V \subset \mathbb{R}^{d}$ be open sets and let $f: U \times V \rightarrow \mathbb{R}^{d}$ be a $C^{1}$-map such that the maps $f(\cdot, y): U \rightarrow f(U, y)$ and $f(x, \cdot): V \rightarrow f(x, V)$ are diffeomorphisms for all $(x, y) \in U \times V$. Denote by $D_{1} f$ and $D_{2} f$ the derivatives of $f(\cdot, y)$ and $f(x, \cdot)$, respectively. We assume that there exists a constant $C_{u}>0$ such that

$$
\left\|D_{i} f(x, y)\right\|,\left\|\left(D_{i} f(x, y)\right)^{-1}\right\| \leq C_{u}
$$

for all $(x, y) \in U \times V$ and $i=1,2$.

Let $\sigma$ be a Radon probability measure on $U$ which is not purely singular with respect to the Lebesgue measure $\mathcal{L}$. We consider the probability space $\left(U^{\mathbb{N}}, \mathcal{F}, \mathbb{P}\right)$ which is the completion of the infinite product of $(U, \mathcal{B}(U), \sigma)$, where $\mathcal{B}(U)$ is the Borel $\sigma$-algebra on $U$. Assuming that $\mathbf{A}=\left(A_{n}\right)_{n \in \mathbb{N}}$ is a sequence of subsets of $V$, define for all $\mathbf{x} \in U^{\mathbb{N}}$ a random covering set $\mathbf{E}(\mathbf{x}, \mathbf{A})$ by

$$
\mathbf{E}(\mathbf{x}, \mathbf{A}):=\limsup _{n \rightarrow \infty} f\left(x_{n}, A_{n}\right)=\bigcap_{n=1}^{\infty} \bigcup_{k=n}^{\infty} f\left(x_{k}, A_{k}\right) .
$$

Now we can finally present our main theorem.

THEOREM 1.1. Let $f: U \times V \rightarrow \mathbb{R}^{d}$ be as above and let $\Delta \subset V$ be compact. Assume that $\mathbf{A}=\left(A_{n}\right)_{n \in \mathbb{N}}$ is a sequence of nonempty subsets of $\Delta$. Then: 
(a) $\operatorname{dim}_{H} \mathbf{E}(\mathbf{x}, \mathbf{A}) \leq t_{0}$ (A) for all $\mathbf{x} \in U^{\mathbb{N}}$.

(b) $\operatorname{dim}_{\mathrm{H}} \mathbf{E}(\mathbf{x}, \mathbf{A}) \geq s_{0}(\mathbf{A})$ for $\mathbb{P}$-almost all $\mathbf{x} \in U^{\mathbb{N}}$ provided that $\mathbf{A}$ is a sequence of Lebesgue measurable sets.

(c) $s_{0}(\mathbf{A})=t_{0}(\mathbf{A})$ provided that $\mathbf{A}$ is a sequence of Lebesgue measurable sets with positive Lebesgue density.

(d) $\operatorname{dim}_{P} \mathbf{E}(\mathbf{x}, \mathbf{A})=d$ for $\mathbb{P}$-almost all $\mathbf{x} \in U^{\mathbb{N}}$ provided that $A_{n}$ are Lebesgue measurable and $\mathcal{L}\left(A_{n}\right)>0$ for infinitely many $n \in \mathbb{N}$.

It follows immediately from Theorem 1.1(d) that the upper box counting dimension of $\mathbf{E}(\mathbf{x}, \mathbf{A})$ equals $d$ almost surely. From the proof of Theorem 1.1(d), we see that $\mathbf{E}(\mathbf{x}, \mathbf{A})$ is almost surely dense in a set of positive Lebesgue measure. Therefore, also the lower box counting dimension equals $d$ almost surely. As a corollary of Theorem 1.1, we obtain the following dimension result for random covering sets in Riemann manifolds. Note that in Corollary 1.2 the quantities $s_{0}(\mathbf{A})$ and $t_{0}(\mathbf{A})$ are defined as in (1.7) and (1.8) by using the distance function induced by the Riemann metric and by replacing $\mathcal{L}$ by the Riemann volume.

COROllary 1.2. Let $\mathbf{K}, \mathbf{M}$ and $\mathbf{N}$ be d-dimensional Riemann manifolds. Assume that $f: \mathbf{K} \times \mathbf{M} \rightarrow \mathbf{N}$ is a $C^{1}$-map such that $f(x, \cdot)$ and $f(\cdot, y)$ are local diffeomorphisms satisfying (1.13). Let $\Delta \subset \mathbf{M}$ be compact and let $\mathbf{A}=\left(A_{n}\right)_{n \in \mathbb{N}}$ be a sequence of subsets of $\Delta$. Suppose that $\sigma$ is a Radon probability measure on $\mathbf{K}$ such that it is not purely singular with respect to the Riemann volume on $\mathbf{K}$. Then the statements (a)-(d) of Theorem 1.1 are valid.

As mentioned earlier, choosing $\mathbf{K}=\mathbf{M}=\mathbf{N}=\mathbb{T}^{d}, f(x, y)=x+y$ and $\sigma=\mathcal{L}$, we recover the previously mentioned setting in $\mathbb{T}^{d}$. The assumption that the generating sets are subsets of a compact set $\Delta$ is needed, for example, to guarantee that $\mathbf{E}(\mathbf{x}, \mathbf{A})$ is nonempty. A natural class of generating sets $\mathbf{A}$ which satisfy the assumptions of Theorem 1.1 and to which the earlier known results are not applicable are regular Cantor sets having positive Lebesgue measure. For the role of other assumptions in Theorem 1.1, we refer to Section 7 where, among other things, sharpness of our results will be discussed. Theorem 1.1 has a refinement concerning the Hausdorff measures of $\mathbf{E}(\mathbf{x}, \mathbf{A})$ with respect to doubling gauge functions. The exact statement of this result is given in Section 8 .

The paper is organised as follows: We begin with technical auxiliary results in Section 2. In Section 3, we prove items (a) and (c) of Theorem 1.1. In Section 4, we introduce a new concept called minimal regular energy and show how it can be used to estimate Hausdorff dimensions of random covering sets. Section 5 is dedicated to the proof of Theorem 1.1(b) whereas the statement (d) is handled in Section 6. In Section 7, we explain how Corollary 1.2 follows from Theorem 1.1 and illustrate by examples the role of the assumptions and the sharpness of Theorem 1.1. In the last section, we give further generalisations of Theorem 1.1 and some remarks. For example, we present some results concerning Hausdorff measures of random covering sets with respect to general gauge functions. 
2. Auxiliary results. In this section, we prove technical lemmas which will be needed in Sections 3-6. When studying random covering sets in the torus, one often utilises the simple fact that $u \in x+E$ if and only if $x \in u-E$ for every $E \subset \mathbb{T}^{d}$. In the nonlinear setting, given a parameterised family of diffeomorphisms $W_{x}$, we attempt to find a parameterised family of diffeomorphisms $X_{u}$ such that $u \in W_{x}(E)$ if and only if $x \in X_{u}(E)$. It is easy to see that the linearised local version of this problem has a solution and, therefore, this should be the case for the original nonlinear problem as well. In order to state this result formally, we need the following notation.

Definition 2.1. Let $U \subset \mathbb{R}^{d}$ be open. A $C^{1}$-map $W: U \times \mathbb{R}^{d} \rightarrow \mathbb{R}^{d}$ is said to be a uniform local bidiffeomorphism, if there exist $r_{0}>0, y_{0} \in \mathbb{R}^{d}$ and a constant $C>0$ such that, for all $x \in U$ and $y \in B\left(y_{0}, r_{0}\right)$, the maps $W(x, \cdot): B\left(y_{0}, r_{0}\right) \rightarrow W\left(x, B\left(y_{0}, r_{0}\right)\right)$ and $W(\cdot, y): U \rightarrow W(U, y)$ are uniform diffeomorphisms, that is, diffeomorphisms satisfying

$$
\left\|D_{i} W(x, y)\right\|,\left\|\left(D_{i} W(x, y)\right)^{-1}\right\| \leq C
$$

for all $x \in U, y \in B\left(y_{0}, r_{0}\right)$ and $i=1,2$, where $D_{1} W$ and $D_{2} W$ denote the derivatives of $W(\cdot, y)$ and $W(x, \cdot)$, respectively. A uniform local bidiffeomorphism $W$ generates a parameterised family of uniform diffeomorphisms $W_{x}: B\left(y_{0}, r_{0}\right) \rightarrow$ $W_{x}\left(B\left(y_{0}, r_{0}\right)\right), x \in U$, by the formula $W_{x}(y):=W(x, y)$.

LEMMA 2.2. Let $W_{x}: B\left(y_{0}, r_{0}\right) \rightarrow W_{x}\left(B\left(y_{0}, r_{0}\right)\right), x \in U$, be a parameterised family of uniform diffeomorphisms generated by a uniform local bidiffeomorphism $W: U \times \mathbb{R}^{d} \rightarrow \mathbb{R}^{d}$. Then there exists a parameterised family of uniform diffeomorphisms $X_{z}: V_{z} \rightarrow X_{z}\left(V_{z}\right)$ where $z \in W\left(U, B\left(y_{0}, r_{0}\right)\right)$ and $V_{z} \subset B\left(y_{0}, r_{0}\right)$ is open such that, for all $A \subset B\left(y_{0}, r_{0}\right)$, we have

$$
z \in W_{x}(A) \quad \text { if and only if } x \in X_{z}\left(A \cap V_{z}\right) .
$$

Furthermore,

$$
\left\|D X_{z}(y)\right\|,\left\|\left(D X_{z}(y)\right)^{-1}\right\| \leq C^{2}
$$

for all $z \in W\left(U, B\left(y_{0}, r_{0}\right)\right)$ and $y \in V_{z}$. Here, $C$ is as in Definition 2.1.

ProOF. Since, for all $z \in W\left(U, B\left(y_{0}, r_{0}\right)\right)$, the set $U^{z}:=\{x \in U: z \in$ $\left.W\left(x, B\left(y_{0}, r_{0}\right)\right)\right\}$ is open and nonempty, we may define a map $R^{z}: U^{z} \rightarrow B\left(y_{0}, r_{0}\right)$ by $R^{z}(x):=T_{x}(z)$, where $T_{x}:=W(x, \cdot)^{-1}$. That is,

$$
W\left(x, R^{z}(x)\right)=W\left(x, T_{x}(z)\right)=z .
$$

Consider $z \in W\left(U, B\left(y_{0}, r_{0}\right)\right)$. We show that $R^{z}: U^{z} \rightarrow R^{z}\left(U^{z}\right)$ is a uniform diffeomorphism. If $x, u \in U^{z}$ satisfy $R^{z}(x)=R^{z}(u)$, then $T_{x}(z)=T_{u}(z)=y$ for some $y \in B\left(y_{0}, r_{0}\right)$ and, therefore, $W(x, y)=z=W(u, y)$. Thus $x=u$, 
implying that $R^{z}$ is injective. Since $W\left(x, T_{x}(z)\right)=z$ for all $x \in U^{z}$, we have $D_{1} W\left(x, T_{x}(z)\right)+D_{2} W\left(x, T_{x}(z)\right) \circ D_{x} T_{x}(z)=0$, giving

$$
D R^{z}(x)=D_{x} T_{x}(z)=-\left(D_{2} W\left(x, T_{x}(z)\right)\right)^{-1} \circ D_{1} W\left(x, T_{x}(z)\right) .
$$

This implies

$$
\left\|D R^{z}(x)\right\|,\left\|\left(D R^{z}(x)\right)^{-1}\right\| \leq C^{2}
$$

for all $z \in W\left(U, B\left(y_{0}, r_{0}\right)\right)$ and $x \in U^{z}$. Observing that, for all $A \subset B\left(y_{0}, r_{0}\right)$ and $x \in U$,

$$
\begin{aligned}
z \in W(x, A) & \Longleftrightarrow T_{x}(z) \in A \quad \Longleftrightarrow \quad R^{z}(x) \in A \\
& \Longleftrightarrow x \in\left(R^{z}\right)^{-1}(A),
\end{aligned}
$$

we may define $V_{z}:=R^{z}\left(U^{z}\right)$ and $X_{z}:=\left(R^{z}\right)^{-1}$. The claim (2.2) follows from (2.3).

For every $A \subset \mathbb{R}^{d}$ and $\delta>0$, let

$$
\bar{V}_{\delta}(A):=\left\{x \in \mathbb{R}^{d}: \operatorname{dist}(x, A) \leq \delta\right\}
$$

be the closed $\delta$-neighbourhood of $A$. Here, $\operatorname{dist}(x, A):=\inf \{|x-a|: a \in A\}$ is the distance between $x$ and $A$. According to the next lemma, using the notation of Lemma 2.2, for each Lebesgue measurable set $F \subset \mathbb{R}^{d}$, the Lebesgue measure of $F \cap W_{x}(A)$ is close to that of $W_{x}(A)$ for most points $x \in F$ provided that $A$ is a subset of a sufficiently small ball.

LEMMA 2.3. Let $U \subset \mathbb{R}^{d}, r_{0}>0, y_{0} \in \mathbb{R}^{d}$ and $W: U \times \mathbb{R}^{d} \rightarrow \mathbb{R}^{d}$ be as in Definition 2.1. Assume that $W_{x}\left(y_{0}\right)=x$ for all $x \in U$ and $F \subset U$ is Lebesgue measurable. Then, for every $\varepsilon>0$, there is $\delta=\delta(F, \varepsilon)>0$ such that, for all Lebesgue measurable sets $A \subset B\left(y_{0}, \delta\right)$, we have

$$
\mathcal{L}\left(\left\{x \in F: \mathcal{L}\left(F \cap W_{x}(A)\right) \geq(1-\varepsilon) \mathcal{L}\left(W_{x}(A)\right)\right\}\right) \geq(1-\varepsilon) \mathcal{L}(F) .
$$

ProOF. We start by proving that $x \mapsto \mathcal{L}\left(F \cap W_{x}(A)\right)$ is a Borel map. Assume first that $F$ and $A$ are compact. Since $\mathcal{L}$ is a Radon measure, we have $\mathcal{L}(E)=$ $\lim _{\delta \rightarrow 0} \mathcal{L}\left(\bar{V}_{\delta}(E)\right)$ for all compact sets $E$. This, in turn, implies that the function $E \mapsto \mathcal{L}(E)$, defined for compact sets, is upper semicontinuous. Moreover, the fact that the map $E \mapsto E \cap A$ is upper semicontinuous for compact sets $A \subset \mathbb{R}^{d}$ (for the definition of upper semicontinuity in this context see [38], page 200) implies that the map $x \mapsto \mathcal{L}\left(F \cap W_{x}(A)\right)$ is upper semicontinuous and, therefore, a Borel map.

Assume now that $F$ and $A$ are Lebesgue measurable. Since $\mathcal{L}$ is inner regular, that is, $\mathcal{L}(E)=\sup \{\mathcal{L}(C): C \subset E, C$ is compact $\}$ for all Lebesgue measurable sets $E \subset \mathbb{R}^{d}$, we may choose increasing sequences $\left(F_{i}\right)_{i \in \mathbb{N}}$ and $\left(A_{j}\right)_{j \in \mathbb{N}}$ of compact 
sets such that $F_{i} \subset F, A_{j} \subset A, \lim _{i \rightarrow \infty} \mathcal{L}\left(F_{i}\right)=\mathcal{L}(F)$ and $\lim _{j \rightarrow \infty} \mathcal{L}\left(W_{x}\left(A_{j}\right)\right)=$ $\mathcal{L}\left(W_{x}(A)\right)$ for all $x \in U$. In particular,

$$
\lim _{j \rightarrow \infty} \lim _{i \rightarrow \infty} \mathcal{L}\left(F_{i} \cap W_{x}\left(A_{j}\right)\right)=\mathcal{L}\left(F \cap W_{x}(A)\right)
$$

for all $x \in U$ and, therefore, the map $x \mapsto \mathcal{L}\left(F \cap W_{x}(A)\right)$ is Borel measurable. It follows that all the sets we encounter in the proof below are Lebesgue measurable.

First, we prove (2.5) for compact sets $F$. Clearly, we may assume that $\mathcal{L}(F)>0$. Note that (2.5) is equivalent to

$$
\mathcal{L}\left(\left\{x \in F: \mathcal{L}\left(F^{c} \cap W_{x}(A)\right)>\varepsilon \mathcal{L}\left(W_{x}(A)\right)\right\}\right)<\varepsilon \mathcal{L}(F),
$$

where the complement of a set $E$ is denoted by $E^{c}$. Now suppose that (2.6) is not true. Then there exists $\varepsilon>0$ such that, for all $\delta>0$, there is a measurable set $A \subset B\left(y_{0}, \delta\right)$ with $\mathcal{L}(A)>0$ satisfying $\mathcal{L}(\Lambda) \geq \varepsilon \mathcal{L}(F)$, where

$$
\Lambda:=\left\{x \in F: \mathcal{L}\left(F^{c} \cap W_{x}(A)\right)>\varepsilon \mathcal{L}\left(W_{x}(A)\right)\right\} .
$$

Suppose that $z \in W_{x}(A)$. Since $W_{x}\left(y_{0}\right)=x$ for all $x \in U$, we have $|z-x| \leq$ $C_{2} \delta=: \tilde{\delta}$. Denoting the characteristic function of a set $E$ by $\chi_{E}$, we obtain by Fubini's theorem that

$$
\begin{aligned}
\int_{\Lambda} \mathcal{L} & \left.F^{c} \cap W_{x}(A)\right) d \mathcal{L}(x) \\
\quad \leq & \int_{F} \mathcal{L}\left(F^{c} \cap W_{x}(A)\right) d \mathcal{L}(x) \\
& =\iint \chi_{F}(x) \chi_{W_{x}(A)}(z) \chi_{F^{c}}(z) d \mathcal{L}(z) d \mathcal{L}(x) \\
& =\iint \chi_{F}(x) \chi_{W_{x}(A)}(z) \chi_{\bar{V}_{\tilde{\delta}}(F) \backslash F}(z) d \mathcal{L}(z) d \mathcal{L}(x) \\
& =\int_{\bar{V}_{\tilde{\delta}}(F) \backslash F} \int_{F} \chi_{W_{x}(A)}(z) d \mathcal{L}(x) d \mathcal{L}(z) .
\end{aligned}
$$

From Lemma 2.2, we deduce that $z \in W_{x}(A)$ if and only if $x \in X_{z}\left(A \cap V_{z}\right)$. Furthermore, $\mathcal{L}\left(X_{z}\left(A \cap V_{z}\right)\right) \leq C^{2 d} \mathcal{L}(A)$ by (2.2). Thus

$$
\int_{\Lambda} \mathcal{L}\left(F^{c} \cap W_{x}(A)\right) d \mathcal{L}(x) \leq C^{2 d} \mathcal{L}(A) \mathcal{L}\left(\bar{V}_{\tilde{\delta}}(F) \backslash F\right) .
$$

On the other hand, since $W_{x}$ is a uniform diffeomorphism, $\mathcal{L}\left(W_{x}(A)\right) \geq$ $C^{-d} \mathcal{L}(A)$ for all $x \in U$. Combining this with the definition of $\Lambda$, inequality (2.8) 
and the fact that $\mathcal{L}(\Lambda) \geq \varepsilon \mathcal{L}(F)$, we obtain

$$
\begin{aligned}
\int_{\Lambda} \mathcal{L}\left(F^{c} \cap W_{x}(A)\right) d \mathcal{L}(x) \\
\geq \varepsilon \int_{\Lambda} \mathcal{L}\left(W_{x}(A)\right) d \mathcal{L}(x) \\
=\varepsilon \int_{\Lambda} \int \chi_{W_{x}(A)}(z) d \mathcal{L}(z) d \mathcal{L}(x) \\
\geq \varepsilon \int_{\Lambda} \int \chi_{W_{x}(A)}(z) \chi_{F}(z) d \mathcal{L}(z) d \mathcal{L}(x) \\
=\varepsilon \int_{\Lambda} \int \chi_{W_{x}(A)}(z) d \mathcal{L}(z) d \mathcal{L}(x) \\
-\varepsilon \int_{\Lambda} \int \chi_{W_{x}(A)}(z) \chi_{F^{c}}(z) d \mathcal{L}(z) d \mathcal{L}(x) \\
\geq C^{-d} \varepsilon \mathcal{L}(A) \mathcal{L}(\Lambda)-\varepsilon \int_{\Lambda} \int \chi_{W_{x}(A)}(z) \chi_{F^{c}}(z) d \mathcal{L}(z) d \mathcal{L}(x) \\
\geq \varepsilon \mathcal{L}(A)\left(C^{-d} \varepsilon \mathcal{L}(F)-C^{2 d} \mathcal{L}\left(\bar{V}_{\tilde{\delta}}(F) \backslash F\right)\right) .
\end{aligned}
$$

Since $F$ is compact, $\mathcal{L}(F)=\lim _{i \rightarrow \infty} \mathcal{L}\left(\bar{V}_{\frac{1}{i}}(F)\right)$ and, therefore, for every $\tilde{\varepsilon}>0$, there is $\delta>0$ such that $\mathcal{L}\left(\bar{V}_{\tilde{\delta}}(F) \backslash F\right)<\tilde{\varepsilon} \mathcal{L}(F)$. Hence, (2.9) contradicts (2.8), completing the proof of (2.5) for compact sets $F$.

For a Lebesgue measurable set $F$, choose a compact set $K \subset F$ satisfying $\mathcal{L}(K) \geq(1-\varepsilon) \mathcal{L}(F)$. Then

$$
\begin{aligned}
\mathcal{L}(\{x & \left.\left.\in F: \mathcal{L}\left(F \cap W_{x}(A)\right) \geq(1-\varepsilon)^{2} \mathcal{L}\left(W_{x}(A)\right)\right\}\right) \\
& \geq \mathcal{L}\left(\left\{x \in K: \mathcal{L}\left(K \cap W_{x}(A)\right) \geq(1-\varepsilon) \mathcal{L}\left(W_{x}(A)\right)\right\}\right) \\
& \geq(1-\varepsilon) \mathcal{L}(K) \geq(1-\varepsilon)^{2} \mathcal{L}(F),
\end{aligned}
$$

completing the proof of (2.5).

The last lemma of this section is a counterpart of Lemma 2.3 for energies of sets.

LEMMA 2.4. Let $U \subset \mathbb{R}^{d}, r_{0}>0, y_{0} \in \mathbb{R}^{d}$ and $W: U \times \mathbb{R}^{d} \rightarrow \mathbb{R}^{d}$ be as in Definition 2.1. Assume that $W_{x}\left(y_{0}\right)=x$ for all $x \in U$. Let $F_{1}, F_{2} \subset U$ be bounded Lebesgue measurable sets and let $0 \leq t<d$. Then, for every $\varepsilon>0$, there exists $\delta_{1}=\delta_{1}\left(F_{1}, F_{2}, \varepsilon\right)>0$ such that

$$
\begin{aligned}
& \iint_{F_{1} \times F_{2}} \iint_{W_{x_{1}}\left(A_{1}\right) \times W_{x_{2}}\left(A_{2}\right)}\left|u_{1}-u_{2}\right|^{-t} d \mathcal{L}\left(u_{1}\right) d \mathcal{L}\left(u_{2}\right) d \mathcal{L}\left(x_{1}\right) d \mathcal{L}\left(x_{2}\right) \\
& \quad \leq(1+\varepsilon) \iint_{F_{1} \times F_{2}} \mathcal{L}\left(W_{x_{1}}\left(A_{1}\right)\right) \mathcal{L}\left(W_{x_{2}}\left(A_{2}\right)\right)\left|x_{1}-x_{2}\right|^{-t} d \mathcal{L}\left(x_{1}\right) d \mathcal{L}\left(x_{2}\right),
\end{aligned}
$$

provided that $A_{1}, A_{2} \subset B\left(y_{0}, \delta_{1}\right)$ are Lebesgue measurable. 
ProOF. Clearly, we may assume that $\mathcal{L}\left(F_{1}\right)>0$ and $\mathcal{L}\left(F_{2}\right)>0$. Let $R>1$ be such that $F_{1}, F_{2} \subset B(0, R-1)$. Then

$$
\begin{aligned}
0 & <\iint_{F_{1} \times F_{2}}\left|u_{1}-u_{2}\right|^{-t} d \mathcal{L}\left(u_{1}\right) d \mathcal{L}\left(u_{2}\right) \\
& \leq \iint_{B(0, R) \times B(0, R)}\left|u_{1}-u_{2}\right|^{-t} d \mathcal{L}\left(u_{1}\right) d \mathcal{L}\left(u_{2}\right)<\infty .
\end{aligned}
$$

It follows that, for every $\tilde{\varepsilon}>0$, there exists $\delta \in \mathbb{R}$ with $0<\delta<1$ such that

$$
\begin{aligned}
& \iint_{D(\delta)}\left|u_{1}-u_{2}\right|^{-t} d \mathcal{L}\left(u_{1}\right) d \mathcal{L}\left(u_{2}\right) \\
& \quad \leq \tilde{\varepsilon} \iint_{F_{1} \times F_{2}}\left|u_{1}-u_{2}\right|^{-t} d \mathcal{L}\left(u_{1}\right) d \mathcal{L}\left(u_{2}\right),
\end{aligned}
$$

where $D(\delta):=\left\{\left(u_{1}, u_{2}\right) \in B(0, R) \times B(0, R):\left|u_{1}-u_{2}\right| \leq \delta\right\}$. Consider $0<$ $\tilde{\varepsilon}<\frac{1}{2}$ and let $\delta>0$ be such that (2.10) is valid. Defining $\delta_{1}:=\frac{1}{4} C^{-1} \tilde{\varepsilon} \delta$, gives $\operatorname{diam} W_{x}\left(B\left(y_{0}, \delta_{1}\right)\right)<\frac{1}{2} \tilde{\varepsilon} \delta$ for all $x \in U$.

Let $A_{1}$ and $A_{2}$ be Lebesgue measurable subsets of $B\left(y_{0}, \delta_{1}\right)$. Recall that $W_{x}\left(y_{0}\right)=x$ for all $x \in U$. Thus, if $u_{i} \in W_{x_{i}}\left(A_{i}\right)$ for $i=1,2$ and $\left|x_{1}-x_{2}\right|>\frac{1}{2} \delta$, we have $\left|u_{1}-u_{2}\right|>(1-2 \tilde{\varepsilon})\left|x_{1}-x_{2}\right|$ and, therefore,

$$
\begin{aligned}
& \int_{\left\{\left(x_{1}, x_{2}\right) \in F_{1} \times F_{2}:\left|x_{1}-x_{2}\right|>\frac{1}{2} \delta\right\}} \iint_{W_{x_{1}}\left(A_{1}\right) \times W_{x_{2}}\left(A_{2}\right)}\left|u_{1}-u_{2}\right|^{-t} d \mathcal{L}\left(u_{1}\right) d \mathcal{L}\left(u_{2}\right) d \mathcal{L}\left(x_{1}\right) d \mathcal{L}\left(x_{2}\right) \\
& \leq(1-2 \tilde{\varepsilon})^{-t} \int_{\left\{\left(x_{1}, x_{2}\right) \in F_{1} \times F_{2}:\left|x_{1}-x_{2}\right|>\frac{1}{2} \delta\right\}}\left|x_{1}-x_{2}\right|^{-t} \\
& \quad \mathcal{L}\left(W_{x_{1}}\left(A_{1}\right)\right) \mathcal{L}\left(W_{x_{2}}\left(A_{2}\right)\right) d \mathcal{L}\left(x_{1}\right) d \mathcal{L}\left(x_{2}\right) \\
& \leq(1-2 \tilde{\varepsilon})^{-t} \\
& \quad \times \iint_{F_{1} \times F_{2}} \mathcal{L}\left(W_{x_{1}}\left(A_{1}\right)\right) \mathcal{L}\left(W_{x_{2}}\left(A_{2}\right)\right)\left|x_{1}-x_{2}\right|^{-t} d \mathcal{L}\left(x_{1}\right) d \mathcal{L}\left(x_{2}\right) .
\end{aligned}
$$

To estimate the remaining part of the integral, we make the change of variables $u_{i}=W_{x_{i}}\left(\tilde{u}_{i}\right)=W\left(x_{i}, \tilde{u}_{i}\right)$ for $i=1,2$. The Jacobians of these coordinate transfor- 
mations are bounded from above by $C^{d}$. By Fubini's theorem,

$$
\begin{aligned}
& \int_{\left\{\left(x_{1}, x_{2}\right) \in F_{1} \times F_{2}:\left|x_{1}-x_{2}\right| \leq \frac{1}{2} \delta\right\}} \\
& \quad \iint_{W_{x_{1}}\left(A_{1}\right) \times W_{x_{2}}\left(A_{2}\right)}\left|u_{1}-u_{2}\right|^{-t} d \mathcal{L}\left(u_{1}\right) d \mathcal{L}\left(u_{2}\right) d \mathcal{L}\left(x_{1}\right) d \mathcal{L}\left(x_{2}\right) \\
& \leq C^{2 d} \iint_{A_{1} \times A_{2}} \int_{\left\{\left(x_{1}, x_{2}\right) \in F_{1} \times F_{2}:\left|x_{1}-x_{2}\right| \leq \frac{1}{2} \delta\right\}}\left|W\left(x_{1}, \tilde{u}_{1}\right)-W\left(x_{2}, \tilde{u}_{2}\right)\right|^{-t} \\
& \quad \times d \mathcal{L}\left(x_{1}\right) d \mathcal{L}\left(x_{2}\right) d \mathcal{L}\left(\tilde{u}_{1}\right) d \mathcal{L}\left(\tilde{u}_{2}\right)=: L .
\end{aligned}
$$

Recall that, by the choice of $\delta_{1}$, we have $\left|W\left(x_{1}, \tilde{u}_{1}\right)-W\left(x_{2}, \tilde{u}_{2}\right)\right| \leq \delta$ provided that $\left|x_{1}-x_{2}\right| \leq \frac{1}{2} \delta$. The fact that, for $i=1,2$, we have $\left|W\left(x_{i}, \tilde{u}_{i}\right)-W\left(x_{i}, y_{0}\right)\right| \leq$ $C \delta_{1}<1$ for all $x_{i} \in F_{i}$ and $\tilde{u}_{i} \in A_{i}$ gives $W\left(x_{i}, \tilde{u}_{i}\right) \in B(0, R)$. Making the change of variables $\tilde{x}_{i}=W\left(x_{i}, \tilde{u}_{i}\right)$ for $i=1,2$ and using the fact that the Jacobians are bounded by $C^{d}$, inequality (2.10) gives

$$
\begin{aligned}
L & \leq C^{4 d} \iint_{A_{1} \times A_{2}} \iint_{D(\delta)}\left|\tilde{x}_{1}-\tilde{x}_{2}\right|^{-t} d \mathcal{L}\left(\tilde{x}_{1}\right) d \mathcal{L}\left(\tilde{x}_{2}\right) d \mathcal{L}\left(\tilde{u}_{1}\right) d \mathcal{L}\left(\tilde{u}_{2}\right) \\
& \leq C^{4 d} \tilde{\varepsilon} \mathcal{L}\left(A_{1}\right) \mathcal{L}\left(A_{2}\right) \iint_{F_{1} \times F_{2}}\left|\tilde{x}_{1}-\tilde{x}_{2}\right|^{-t} d \mathcal{L}\left(\tilde{x}_{1}\right) d \mathcal{L}\left(\tilde{x}_{2}\right) \\
& \leq C^{6 d} \tilde{\varepsilon} \iint_{F_{1} \times F_{2}} \mathcal{L}\left(W_{x_{1}}\left(A_{1}\right)\right) \mathcal{L}\left(W_{x_{2}}\left(A_{2}\right)\right)\left|\tilde{x}_{1}-\tilde{x}_{2}\right|^{-t} d \mathcal{L}\left(\tilde{x}_{1}\right) d \mathcal{L}\left(\tilde{x}_{2}\right)
\end{aligned}
$$

Combining (2.11) and (2.12) gives the claim.

3. Upper bound for Hausdorff dimension. In this section, we prove claims (a) and (c) in Theorem 1.1. We begin with the following observation.

LEMMA 3.1. Let $\left(E_{n}\right)_{n \in \mathbb{N}}$ be a sequence of subsets of $\mathbb{R}^{d}$. Then

$$
\operatorname{dim}_{\mathrm{H}}\left(\limsup _{n \rightarrow \infty} E_{n}\right) \leq \inf \left\{t \geq 0: \sum_{n=1}^{\infty} \mathcal{H}_{\infty}^{t}\left(E_{n}\right)<\infty\right\} .
$$

Proof. For $0 \leq s<\infty$ and $0<\delta<\infty$, we denote by $\mathcal{H}^{s}$ and $\mathcal{H}_{\delta}^{s}$ the $s$-dimensional Hausdorff measure and $\delta$-measure, respectively. Let $t>0$ with $\sum_{n=1}^{\infty} \mathcal{H}_{\infty}^{t}\left(E_{n}\right)<\infty$. For the purpose of proving the claim, it suffices to show that $\operatorname{dim}_{\mathrm{H}}\left(\lim \sup _{n \rightarrow \infty} E_{n}\right) \leq t$. In what follows, we prove a slightly stronger result that $\mathcal{H}^{t}\left(\limsup _{n \rightarrow \infty} E_{n}\right)=0$.

Let $\varepsilon>0$ and let $N \in \mathbb{N}$ so that $\sum_{n=N}^{\infty} \mathcal{H}_{\infty}^{t}\left(E_{n}\right)<\frac{\varepsilon^{t}}{2}$. For every $n \geq N$ and $k \in \mathbb{N}$, we choose $U_{n, k} \subset \mathbb{R}^{d}$ such that $\bigcup_{k=1}^{\infty} U_{n, k} \supset E_{n}$ for all $n \geq N$ and $\sum_{n=N}^{\infty} \sum_{k=1}^{\infty}\left(\operatorname{diam} U_{n, k}\right)^{t} \leq \varepsilon^{t}$. Clearly, diam $U_{n, k} \leq \varepsilon$ and, therefore,

$$
\mathcal{H}_{\varepsilon}^{t}\left(\limsup _{n \rightarrow \infty} E_{n}\right) \leq \mathcal{H}_{\varepsilon}^{t}\left(\bigcup_{n=N}^{\infty} E_{n}\right) \leq \sum_{n=N}^{\infty} \sum_{k=1}^{\infty}\left(\operatorname{diam} U_{n, k}\right)^{t} \leq \varepsilon^{t} .
$$


As $\varepsilon$ can be arbitrarily small, we have $\mathcal{H}^{t}\left(\limsup _{n \rightarrow \infty} E_{n}\right)=0$, which completes the proof.

Proof of Theorem 1.1(A). The inequality $\operatorname{dim}_{\mathrm{H}} \mathbf{E}(\mathbf{x}, \mathbf{A}) \leq t_{0}(\mathbf{A})$ follows directly from Lemma 3.1, using a simple observation that $\mathcal{H}_{\infty}^{t}(f(x, E)) \leq$ $\left(C_{u}\right)^{t} \mathcal{H}_{\infty}^{t}(E)$ for all $x \in U$ and $E \subset V$, where $C_{u}$ is the constant appearing in (1.13).

The rest of this section is devoted to proving that $s_{0}(\mathbf{A})=t_{0}(\mathbf{A})$ under the assumptions of Theorem 1.1(c), where $s_{0}(\mathbf{A})$ and $t_{0}(\mathbf{A})$ are as in (1.8) and (1.7), respectively. We start by proving that $s_{0}(\mathbf{A}) \leq t_{0}(\mathbf{A})$.

LEMMA 3.2. Let $E \subset \mathbb{R}^{d}$ be a Lebesgue measurable set. For all $t \geq 0$, we have $\mathcal{H}_{\infty}^{t}(E) \geq G_{t}(E)$. In particular, for every sequence $\mathbf{A}:=\left(A_{n}\right)_{n \in \mathbb{N}}$ of Lebesgue measurable subsets of $\mathbb{R}^{d}$, we have $s_{0}(\mathbf{A}) \leq t_{0}(\mathbf{A})$.

Proof. We may assume that $\mathcal{L}(E)>0$ and $\mathcal{H}_{\infty}^{t}(E)<\infty$. Let $\varepsilon>0$. We choose disjoint Borel sets $E_{n}, \quad n \in \mathbb{N}$, such that $\bigcup_{n=1}^{\infty} E_{n} \supset E$ and $\sum_{n=1}^{\infty}\left(\operatorname{diam} E_{n}\right)^{t}<\mathcal{H}_{\infty}^{t}(E)+\varepsilon$. Notice that, for all $n \in \mathbb{N}$,

$I_{t}\left(E \cap E_{n}\right)=\iint_{\left(E \cap E_{n}\right) \times\left(E \cap E_{n}\right)}|x-y|^{-t} d \mathcal{L}(x) d \mathcal{L}(y) \geq\left(\operatorname{diam} E_{n}\right)^{-t} \mathcal{L}\left(E \cap E_{n}\right)^{2}$.

It follows that

$$
I_{t}(E) \geq \sum_{n=1}^{\infty} I_{t}\left(E \cap E_{n}\right) \geq \sum_{n=1}^{\infty}\left(\operatorname{diam} E_{n}\right)^{-t} \mathcal{L}\left(E \cap E_{n}\right)^{2} .
$$

From (3.1) and the Cauchy-Schwarz inequality, we obtain

$$
\begin{aligned}
\left(\sum_{n=1}^{\infty}\left(\operatorname{diam} E_{n}\right)^{t}\right) I_{t}(E) & \geq\left(\sum_{n=1}^{\infty}\left(\operatorname{diam} E_{n}\right)^{t}\right)\left(\sum_{n=1}^{\infty}\left(\operatorname{diam} E_{n}\right)^{-t} \mathcal{L}\left(E \cap E_{n}\right)^{2}\right) \\
& \geq\left(\sum_{n=1}^{\infty} \mathcal{L}\left(E \cap E_{n}\right)\right)^{2}=\mathcal{L}(E)^{2},
\end{aligned}
$$

which implies that $\sum_{n=1}^{\infty}\left(\operatorname{diam} E_{n}\right)^{t} \geq g_{t}(E)$ [see (1.4)]. Hence, $\mathcal{H}_{\infty}^{t}(E)+\varepsilon>$ $g_{t}(E)$. Letting $\varepsilon$ tend to zero, gives $\mathcal{H}_{\infty}^{t}(E) \geq g_{t}(E)$. As $\mathcal{H}_{\infty}^{t}(\cdot)$ is a monotone increasing function, we conclude that $\mathcal{H}_{\infty}^{t}(E) \geq G_{t}(E)$. According to this inequality, we have $s_{0}(\mathbf{A}) \leq t_{0}(\mathbf{A})$ for every sequence $\mathbf{A}$ of Lebesgue measurable subsets of $\mathbb{R}^{d}$.

REMARK 3.3. The following extension of Lemma 3.2 can be proven with the same argument: if $\mu$ is a finite Borel measure supported on $E$ and $t \geq 0$, we have

$$
\mathcal{H}_{\infty}^{t}(E) \geq \frac{\mu(E)^{2}}{\iint_{E \times E}|x-y|^{-t} d \mu(x) d \mu(y)} .
$$


We proceed by estimating $\mathcal{H}_{\infty}^{t}(E)$ from above by means of $G_{t}(E)$. Our estimate is based on a technical result stated in Proposition 3.8. In what follows, the restriction of a measure $\mu$ to a set $E \subset \mathbb{R}^{d}$ is denoted by $\left.\mu\right|_{E}$, that is, $\left.\mu\right|_{E}(F):=\mu(E \cap F)$ for all $F \subset \mathbb{R}^{d}$. For a Radon measure $\mu$ on $\mathbb{R}^{d}$ and $0<s<d$, let

$$
I_{S}(\mu):=\iint|x-y|^{-s} d \mu(x) d \mu(y)
$$

be the $s$-energy of $\mu$. Given a Borel set $E \subset \mathbb{R}^{d}$, let $\mathcal{P}(E)$ be the space of Borel probability measures supported on $E$, and let $E^{+}$be the set of points in $E$ having positive Lebesgue density, that is,

$$
E^{+}:=\left\{x \in E: \liminf _{r \rightarrow 0} \frac{\mathcal{L}(E \cap B(x, r))}{\mathcal{L}(B(x, r))}>0\right\} .
$$

We denote by $\bar{E}$ the closure of a set $E \subset \mathbb{R}^{d}$, by $\bar{B}(0,1) \subset \mathbb{R}^{d}$ the closed unit ball centred at the origin and by $\mathcal{C}(\bar{B}(0,1))$ the family of continuous maps from $\bar{B}(0,1)$ to $\mathbb{R}$.

We continue by verifying several elementary lemmas.

LEMMA 3.4. Letting $s>0$, the mapping $\eta \mapsto I_{S}(\eta)$ is lower semicontinuous on $\mathcal{P}(\bar{B}(0,1))$, when $\mathcal{P}(\bar{B}(0,1))$ is equipped with the weak-star topology.

PROOF. The result is well known (see, e.g., [40], (1.4.5)) and follows from the fact that the mapping $(x, y) \mapsto|x-y|^{-s}$ is nonnegative and lower semicontinuous on $\mathbb{R}^{d} \times \mathbb{R}^{d}$.

LEMMA 3.5. Let $\eta \in \mathcal{P}(\bar{B}(0,1))$. Suppose that $\left(F_{n}\right)_{n \in \mathbb{N}}$ is a sequence of Borel subsets of $\bar{B}(0,1)$ satisfying $\lim _{n \rightarrow \infty} \eta\left(F_{n}\right)=1$. Then $\eta_{n}:=\left.\eta\left(F_{n}\right)^{-1} \eta\right|_{F_{n}}$ converges to $\eta$ in the weak-star topology as $n$ tends to infinity. Moreover, $\lim _{n \rightarrow \infty} I_{s}\left(\eta_{n}\right)=I_{s}(\eta)$ for all $s>0$.

ProOF. Letting $g \in \mathcal{C}(\bar{B}(0,1))$, we have that

$$
0 \leq \int\left|g-g \chi_{F_{n}}\right| d \eta \leq\left(1-\eta\left(F_{n}\right)\right) \max _{x \in \bar{B}(0,1)}|g(x)| .
$$

From this, it follows that

$$
\lim _{n \rightarrow \infty} \int g d \eta_{n}=\lim _{n \rightarrow \infty} \eta\left(F_{n}\right)^{-1} \int g \chi_{F_{n}} d \eta=\int g d \eta
$$

and, therefore, $\eta_{n}$ converges to $\eta$ in the weak-star topology.

Let $s>0$. By Lemma 3.4, we have $\liminf _{n \rightarrow \infty} I_{S}\left(\eta_{n}\right) \geq I_{S}(\eta)$. Notice that, for all $n \in \mathbb{N}$,

$$
I_{S}\left(\eta_{n}\right)=\eta\left(F_{n}\right)^{-2} \iint_{F_{n} \times F_{n}}|x-y|^{-s} d \eta(x) d \eta(y) \leq \eta\left(F_{n}\right)^{-2} I_{s}(\eta),
$$


which implies that $\limsup _{n \rightarrow \infty} I_{S}\left(\eta_{n}\right) \leq I_{s}(\eta)$. Hence, $\lim _{n \rightarrow \infty} I_{s}\left(\eta_{n}\right)=I_{S}(\eta)$, as desired.

For a Borel set $F \subset \bar{B}(0,1)$ and $s>0$, we recall the notation $I_{s}(F)=I_{s}(\mathcal{L} \mid F)$ from (1.5). For every $k \in \mathbb{N}$, define

$$
\mathcal{Q}_{k}:=\left\{\left[0,2^{-k}\right)^{d}+\alpha: \alpha \in 2^{-k} \mathbb{Z}^{d}\right\} .
$$

Lemma 3.6. Let $F \subset \bar{B}(0,1)$ be a Borel set, and let $0<s<d$. Then, for every $p \in \mathbb{R}$ with $0<p \leq 1$, there exists a Borel set $F_{1} \subset F$ so that $\mathcal{L}\left(F_{1}\right)=$ $p \mathcal{L}(F)$ and $I_{s}\left(F_{1}\right) \leq 2 p^{2} I_{s}(F)$.

Proof. Let $0<p \leq 1$. Write $\mu:=\left.\mathcal{L}\right|_{F}$ and choose a large integer $\ell \in \mathbb{N}$ so that

$$
\left(1+\frac{2 \sqrt{d}}{\ell}\right)^{s}<\frac{3}{2} .
$$

Since $I_{s}(\mu)<\infty$, there is $n \in \mathbb{N}$ such that

$$
\sum_{\substack{Q, Q^{\prime} \in \mathcal{Q}_{n} \\ \text { iist }\left(Q, Q^{\prime}\right)<2^{-n} \ell}} \iint_{Q \times Q^{\prime}}|x-y|^{-s} d \mu(x) d \mu(y)<\frac{1}{2} p^{2} I_{S}(\mu) .
$$

Here, $\operatorname{dist}\left(Q, Q^{\prime}\right)=\inf \left\{|x-y|: x \in Q\right.$ and $\left.y \in Q^{\prime}\right\}$. For each $Q \in \mathcal{Q}_{n}$, construct a Borel subset $\widetilde{Q}$ of $Q \cap F$ such that $\mathcal{L}(\widetilde{Q})=p \mathcal{L}(Q \cap F)$. Defining $F_{1}:=\bigcup_{Q \in \mathcal{Q}_{n}} \widetilde{Q}$, we have $F_{1} \subset F$ and $\mathcal{L}\left(F_{1}\right)=p \mathcal{L}(F)$.

We proceed by showing that $I_{s}\left(F_{1}\right) \leq 2 p^{2} I_{S}(F)$. Set $\eta:=\left.\mathcal{L}\right|_{F_{1}}$. Since $F_{1} \subset F$, inequality (3.4) gives

$$
\sum_{\substack{Q, Q^{\prime} \in \mathcal{Q}_{n} \\ \text { dist }\left(Q, Q^{\prime}\right)<2^{-n} \ell}} \iint_{Q \times Q^{\prime}}|x-y|^{-s} d \eta(x) d \eta(y)<\frac{1}{2} p^{2} I_{s}(\mu) .
$$

The proof will be complete, once we show that

$$
\sum_{\substack{Q, Q^{\prime} \in \mathcal{Q}_{n} \\ \operatorname{dist}\left(Q, Q^{\prime}\right) \geq 2^{-n} \ell}} \iint_{Q \times Q^{\prime}}|x-y|^{-s} d \eta(x) d \eta(y) \leq \frac{3}{2} p^{2} I_{S}(\mu) .
$$

Note that if $Q, Q^{\prime} \in \mathcal{Q}_{n}$ with $\operatorname{dist}\left(Q, Q^{\prime}\right) \geq 2^{-n} \ell, x \in Q$ and $y \in Q^{\prime}$, we obtain

$$
\operatorname{dist}\left(Q, Q^{\prime}\right) \leq|x-y| \leq \operatorname{dist}\left(Q, Q^{\prime}\right)+2 \sqrt{d} 2^{-n}
$$

and, therefore, by (3.3),

$$
\frac{2}{3} \operatorname{dist}\left(Q, Q^{\prime}\right)^{-s} \leq|x-y|^{-s} \leq \operatorname{dist}\left(Q, Q^{\prime}\right)^{-s} .
$$


This, in turn, implies that

$$
\begin{aligned}
& \iint_{Q \times Q^{\prime}}|x-y|^{-s} d \eta(x) d \eta(y) \\
& \quad \leq \operatorname{dist}\left(Q, Q^{\prime}\right)^{-s} \eta(Q) \eta\left(Q^{\prime}\right) \\
& \quad=p^{2} \operatorname{dist}\left(Q, Q^{\prime}\right)^{-s} \mu(Q) \mu\left(Q^{\prime}\right) \leq \frac{3}{2} p^{2} \iint_{Q \times Q^{\prime}}|x-y|^{-s} d \mu(x) d \mu(y) .
\end{aligned}
$$

Summing over $Q, Q^{\prime} \in \mathcal{Q}_{n}$ with $d\left(Q, Q^{\prime}\right) \geq 2^{-n} \ell$, we obtain (3.5) as desired.

The following lemma is a special case of Proposition 3.8.

LEMMA 3.7. Let $E \subset \bar{B}(0,1)$ be a Borel set with $\mathcal{L}(E)>0$, and let $k, m \in \mathbb{N}$. Assume that $E_{0} \subset E$ is a nonempty Borel set such that

$$
\frac{\mathcal{L}(E \cap B(x, r))}{\mathcal{L}(B(x, r))}>\frac{1}{k}
$$

for all $x \in E_{0}$ and $0<r \leq 2^{-m}$. Let $0<s<d$ and $\mu \in \mathcal{P}\left(E_{0}\right)$ with $I_{s}(\mu)<\infty$. Then there is a sequence $\left(F_{n}\right)_{n \in \mathbb{N}}$ of Borel subsets of $E$ with positive Lebesgue measure such that the sequence $\mu_{n}:=\left.\mathcal{L}\left(F_{n}\right)^{-1} \mathcal{L}\right|_{F_{n}}, n \in \mathbb{N}$, converges to $\mu$ in the weak-star topology as $n$ tends to infinity, and $\lim _{n \rightarrow \infty} I_{s}\left(\mu_{n}\right)=I_{S}(\mu)$.

PROOF. We divide the proof into three steps.

Step 1. Construction of $\left(\mu_{n}\right)_{n \in \mathbb{N}}$. For all $n \in \mathbb{N}$, let

$$
\left\{x_{n, 1}, \ldots, x_{n, p_{n}}:\left|x_{n, i}-x_{n, j}\right| \geq 2^{-n} \text { for all } i \neq j\right\}
$$

be a subset of $E_{0}$ with maximal cardinality. Then

$$
E_{0} \subset \bigcup_{i=1}^{p_{n}} B\left(x_{n, i}, 2^{-n}\right) .
$$

For $i=1, \ldots, p_{n}$, we denote by $Q_{n, i}$ the set of points $y \in B\left(x_{n, i}, 2^{-n}\right)$ for which $i$ is the smallest index such that $\left|y-x_{n, i}\right|=\min _{j=1, \ldots, p_{n}}\left|y-x_{n, j}\right|$. Then the sets $Q_{n, i}, i=1, \ldots, p_{n}$, are pairwise disjoint Borel sets satisfying

$$
E_{0} \subset \bigcup_{i=1}^{p_{n}} Q_{n, i}=\bigcup_{i=1}^{p_{n}} B\left(x_{n, i}, 2^{-n}\right) \quad \text { and }
$$

$$
B\left(x_{n, i}, 2^{-n-1}\right) \subset Q_{n, i} \subset B\left(x_{n, i}, 2^{-n}\right) \quad \text { for all } i=1, \ldots, p_{n} .
$$

For all $i=1, \ldots, p_{n}$, define

$$
c_{i}:=\frac{\mu\left(Q_{n, i}\right)}{\mathcal{L}\left(E \cap B\left(x_{n, i}, 2^{-n-2}\right)\right)}
$$


and set $c:=\max _{i=1, \ldots, p_{n}} c_{i}$. Lemma 3.6 implies that, for every $i=1, \ldots, p_{n}$, we can construct a Borel set $F_{n, i}$ such that

$$
\begin{aligned}
F_{n, i} & \subset E \cap B\left(x_{n, i}, 2^{-n-2}\right), \\
\mathcal{L}\left(F_{n, i}\right) & =\frac{c_{i}}{c} \mathcal{L}\left(E \cap B\left(x_{n, i}, 2^{-n-2}\right)\right) \quad \text { and } \\
I_{S}\left(F_{n, i}\right) & \leq \frac{2 c_{i}^{2}}{c^{2}} I_{S}\left(E \cap B\left(x_{n, i}, 2^{-n-2}\right)\right) .
\end{aligned}
$$

By (3.10), the sets $F_{n, i}, i=1, \ldots, p_{n}$, are pairwise disjoint and, moreover,

$$
\operatorname{dist}\left(F_{n, i}, F_{n, j}\right) \geq 2^{-n-1} \quad \text { for } i \neq j .
$$

We complete the construction in step 1 by setting

$$
F_{n}:=\bigcup_{i=1}^{p_{n}} F_{n, i} \quad \text { and } \quad \mu_{n}:=\left.\mathcal{L}\left(F_{n}\right)^{-1} \mathcal{L}\right|_{F_{n}} .
$$

Observe that $\mathcal{L}\left(F_{n}\right)>0$ since $\mathcal{L}(B(x, r) \cap E)>0$ for all $x \in E_{0}$ and $r>0$.

Step 2. Convergence of $\left(\mu_{n}\right)_{n \in \mathbb{N}}$. By (3.9) and (3.11), we have $\mathcal{L}\left(F_{n, i}\right)=$ $c^{-1} \mu\left(Q_{n, i}\right)$ for all $i=1, \ldots, p_{n}$. It follows that

$$
\begin{aligned}
\mathcal{L}\left(F_{n}\right) & =c^{-1} \quad \text { and } \\
\mu_{n}\left(Q_{n, i}\right) & =\mu_{n}\left(F_{n, i}\right)=\mu\left(Q_{n, i}\right)
\end{aligned}
$$

for all $i=1, \ldots, p_{n}$. Let $F \subset \mathbb{R}^{d}$ be a compact set. From (3.15) and the fact that $\operatorname{diam}\left(Q_{n, i}\right) \leq 2 \cdot 2^{-n}$ [see (3.8)], we conclude that, for all $\varepsilon>0$ [recall (2.4)],

$$
\limsup _{n \rightarrow \infty} \mu_{n}(F) \leq \limsup _{n \rightarrow \infty} \sum_{\substack{1 \leq i \leq p_{n} \\ Q_{n, i} \subset F_{\varepsilon}}} \mu_{n}\left(Q_{n, i}\right) \leq \mu\left(\bar{V}_{\varepsilon}(F)\right) .
$$

Combining this with the fact that $\mu(F)=\lim _{\varepsilon \rightarrow 0} \mu\left(\bar{V}_{\varepsilon}(F)\right)$, leads to the conclusion $\limsup _{n \rightarrow \infty} \mu_{n}(F) \leq \mu(F)$. The weak-star convergence now follows from the Portmanteau theorem [38], Theorem 17.20.

Step 3. Convergence of $\left(I_{s}\left(\mu_{n}\right)\right)_{n \in \mathbb{N}}$. Since the sequence $\left(\mu_{n}\right)_{n \in \mathbb{N}}$ converges to $\mu$ in the weak-star topology, Lemma 3.4 gives $\liminf _{n \rightarrow \infty} I_{s}\left(\mu_{n}\right) \geq I_{s}(\mu)$. Hence, for the purpose of proving that $\lim _{n \rightarrow \infty} I_{S}\left(\mu_{n}\right)=I_{S}(\mu)$, it suffices to show that, for every $\varepsilon>0$, there exists $N \in \mathbb{N}$ such that

$$
I_{S}\left(\mu_{n}\right) \leq(1+\varepsilon) I_{S}(\mu)
$$

for all $n \geq N$. Let $\varepsilon>0$ and select $\ell \in \mathbb{N}$ such that

$$
\left(1+\frac{4}{\ell}\right)^{s}<1+\frac{\varepsilon}{2}
$$


Moreover, choose a large integer $N \geq m$ such that, for all $n \geq N$,

$$
\iint_{\left\{(x, y):|x-y| \leq 2^{-n}(\ell+8)\right\}}|x-y|^{-s} d \mu(x) d \mu(y)<\frac{\varepsilon}{4 L} I_{s}(\mu)
$$

where

$$
L:=\max \left\{2^{s}(\ell+8)^{s}, 2 k^{2} I_{s}(B(0,1)) \mathcal{L}(B(0,1))^{-2} 8^{s}\right\} .
$$

Let $n \geq N$ and set $\mathbf{D}_{n}:=\left\{Q_{n, i}: i=1, \ldots, p_{n}\right\}$. Notice that if $Q, Q^{\prime} \in \mathbf{D}_{n}$ with $\operatorname{dist}\left(Q, Q^{\prime}\right) \geq 2^{-n} \ell, x \in Q$ and $y \in Q^{\prime}$, we have, by (3.8), that

$$
\operatorname{dist}\left(Q, Q^{\prime}\right) \leq|x-y| \leq \operatorname{dist}\left(Q, Q^{\prime}\right)+4 \cdot 2^{-n}
$$

and, therefore, by (3.17),

$$
\left(1+\frac{\varepsilon}{2}\right)^{-1} \operatorname{dist}\left(Q, Q^{\prime}\right)^{-s} \leq|x-y|^{-s} \leq \operatorname{dist}\left(Q, Q^{\prime}\right)^{-s} .
$$

Combining this with (3.15), we conclude that

$$
\begin{aligned}
\iint_{Q \times Q^{\prime}}|x-y|^{-s} d \mu_{n}(x) d \mu_{n}(y) \\
\quad \leq \operatorname{dist}\left(Q, Q^{\prime}\right)^{-s} \mu_{n}(Q) \mu_{n}\left(Q^{\prime}\right) \\
\quad=\operatorname{dist}\left(Q, Q^{\prime}\right)^{-s} \mu(Q) \mu\left(Q^{\prime}\right) \\
\quad \leq\left(1+\frac{\varepsilon}{2}\right) \iint_{Q \times Q^{\prime}}|x-y|^{-s} d \mu(x) d \mu(y) .
\end{aligned}
$$

Summing over $Q, Q^{\prime} \in \mathbf{D}_{n}$ with $\operatorname{dist}\left(Q, Q^{\prime}\right) \geq 2^{-n} \ell$, we obtain that

$$
\sum_{\substack{Q, Q^{\prime} \in \mathbf{D}_{n} \\ \operatorname{dist}\left(Q, Q^{\prime}\right) \geq 2^{-n} \ell}} \iint_{Q \times Q^{\prime}}|x-y|^{-s} d \mu_{n}(x) d \mu_{n}(y) \leq\left(1+\frac{\varepsilon}{2}\right) I_{S}(\mu) .
$$

To complete the proof of (3.16), it is sufficient to verify that

$$
\sum_{\substack{Q, Q^{\prime} \in \mathbf{D}_{n} \\ \operatorname{dist}\left(Q, Q^{\prime}\right)<2^{-n} \ell}} \iint_{Q \times Q^{\prime}}|x-y|^{-s} d \mu_{n}(x) d \mu_{n}(y) \leq \frac{\varepsilon}{2} I_{S}(\mu) .
$$

Since $\mu_{n}$ is supported on $F_{n}=\bigcup_{i=1}^{p_{n}} F_{n, i}$, the left-hand side of (3.19) is bounded above by

$$
\sum_{\substack{1 \leq i, j \leq p_{n} \\ \operatorname{dist}\left(F_{n, i}, F_{n, j}\right)<2^{-n}(\ell+4)}} \iint_{F_{n, i} \times F_{n, j}}|x-y|^{-s} d \mu_{n}(x) d \mu_{n}(y)=:(I)+(I I),
$$


where

$$
\begin{aligned}
&(I):=\sum_{\substack{1 \leq i, j \leq p_{n}: i \neq j \\
\operatorname{dist}\left(F_{n, i}, F_{n, j}\right)<2^{-n}(\ell+4)}} \iint_{F_{n, i} \times F_{n, j}}|x-y|^{-s} d \mu_{n}(x) d \mu_{n}(y) \quad \text { and } \\
&(I I):=\sum_{1 \leq i \leq p_{n}} \iint_{F_{n, i} \times F_{n, i}}|x-y|^{-s} d \mu_{n}(x) d \mu_{n}(y) .
\end{aligned}
$$

We proceed by estimating (I) and (II) separately. First, we obtain

$$
\begin{aligned}
& (I) \leq \sum_{1 \leq i, j \leq p_{n}: i \neq j} 2^{(n+1) s} \mu_{n}\left(F_{n, i}\right) \mu_{n}\left(F_{n, j}\right) \quad[\text { by (3.13)] } \\
& \operatorname{dist}\left(F_{n, i}, F_{n, j}\right)<2^{-n}(\ell+4) \\
& =\sum_{1 \leq i, j \leq p_{n}: i \neq j} 2^{(n+1) s} \mu\left(Q_{n, i}\right) \mu\left(Q_{n, j}\right) \quad[\text { by (3.15)] } \\
& \operatorname{dist}\left(F_{n, i}, F_{n, j}\right)<2^{-n}(\ell+4) \\
& \leq \sum_{\substack{Q, Q^{\prime} \in \mathbf{D}_{n} \\
\operatorname{dist}\left(Q, Q^{\prime}\right)<2^{-n}(\ell+4)}} 2^{(n+1) s} \mu(Q) \mu\left(Q^{\prime}\right) \\
& \leq \sum_{\substack{Q, Q^{\prime} \in \mathbf{D}_{n} \\
\operatorname{dist}\left(Q, Q^{\prime}\right)<2^{-n}(\ell+4)}} 2^{s}(\ell+8)^{s} \iint_{Q \times Q^{\prime}}|x-y|^{-s} d \mu(x) d \mu(y) \quad[\text { by (3.8)] } \\
& \leq 2^{s}(\ell+8)^{s} \iint_{\left\{(x, y):|x-y| \leq 2^{-n}(\ell+8)\right\}}|x-y|^{-s} d \mu(x) d \mu(y) \\
& \leq \frac{\varepsilon}{4} I_{S}(\mu) \quad[\text { by (3.18)]. }
\end{aligned}
$$

Set $\alpha:=\mathcal{L}(B(0,1))$ and $\beta:=I_{s}(B(0,1))$. Using the change of variables $\tilde{x}=r x$, it is straightforward to see that

$$
I_{s}(B(x, r))=\mathcal{L}(B(x, r))^{2} \alpha^{-2} r^{-s} \beta
$$

for all $x \in \mathbb{R}^{d}$ and all $r>0$. Therefore,

$$
\begin{array}{rlr}
(I I) & =\sum_{1 \leq i \leq p_{n}} c^{2} I_{S}\left(F_{n, i}\right) \quad[\text { by (3.14) } & \\
& \leq \sum_{1 \leq i \leq p_{n}} 2 c_{i}^{2} I_{S}\left(E \cap B\left(x_{n, i}, 2^{-n-2}\right)\right) \quad[\text { by (3.12)] } \\
& =\sum_{1 \leq i \leq p_{n}} \frac{2 \mu\left(Q_{n, i}\right)^{2} I_{s}\left(E \cap B\left(x_{n, i}, 2^{-n-2}\right)\right)}{\mathcal{L}\left(E \cap B\left(x_{n, i}, 2^{-n-2}\right)\right)^{2}} \quad[\text { by (3.9)] } \\
& \leq \sum_{1 \leq i \leq p_{n}}\left(2 k^{2} \alpha^{-2} \beta 8^{s}\right) \mu\left(Q_{n, i}\right)^{2} 2^{(n-1) s} \quad[\text { by (3.20) and (3.6)] }
\end{array}
$$




$$
\begin{aligned}
& \leq\left(2 k^{2} \alpha^{-2} \beta 8^{s}\right) \sum_{1 \leq i \leq p_{n}} \iint_{Q_{n, i} \times Q_{n, i}}|x-y|^{-s} d \mu(x) d \mu(y) \\
& \leq\left(2 k^{2} \alpha^{-2} \beta 8^{s}\right) \iint_{\left\{(x, y):|x-y| \leq 2 \cdot 2^{-n}\right\}}|x-y|^{-s} d \mu(x) d \mu(y) \\
& \leq \frac{\varepsilon}{4} I_{s}(\mu) \quad[\text { by (3.18)]. }
\end{aligned}
$$

We conclude that $(I)+(I I) \leq \frac{\varepsilon}{2} I_{S}(\mu)$, from which (3.19) follows. This completes the proof of Lemma 3.7.

Now we are ready to state the main technical result of this section.

Proposition 3.8. Let $E \subset \mathbb{R}^{d}$ be a bounded Borel set with $\mathcal{L}(E)>0$, and let $0<s<d$. Assume that $\mu \in \mathcal{P}\left(E^{+}\right)$with $I_{s}(\mu)<\infty$. Then there is a sequence $\left(F_{n}\right)_{n \in \mathbb{N}}$ of Borel subsets of $E^{+}$with positive Lebesgue measure such that the sequence $\mu_{n}:=\left.\mathcal{L}\left(F_{n}\right)^{-1} \mathcal{L}\right|_{F_{n}}, n \in \mathbb{N}$, converges to $\mu$ in the weak-star topology as $n$ tends to infinity, and $\lim _{n \rightarrow \infty} I_{s}\left(\mu_{n}\right)=I_{s}(\mu)$.

ProOF. Without loss of generality, we may assume that $E \subset \bar{B}(0,1)$. For every $k, m \in \mathbb{N}$, define

$$
E_{k, m}:=\left\{x \in E: \frac{\mathcal{L}(E \cap B(x, r))}{\mathcal{L}(B(x, r))}>\frac{1}{k} \text { for all } 0<r \leq 2^{-m}\right\},
$$

and set $E_{k}:=\bigcup_{m=1}^{\infty} E_{k, m}$. Then $E_{k, m} \uparrow E_{k}$ as $m$ tends to infinity and, moreover, $E_{k} \uparrow E^{+}$as $k$ tends to infinity. Choose sufficiently large $k_{0} \in \mathbb{N}$ such that $\mu\left(E_{k_{0}}\right)>$ 0 . For every integer $k \geq k_{0}$, pick $m_{k} \in \mathbb{N}$ such that

$$
\mu\left(E_{k, m_{k}}\right) \geq\left(1-\frac{1}{k}\right) \mu\left(E_{k}\right) .
$$

Since $E_{k} \uparrow E^{+}$as $k$ tends to infinity and $\mu$ is supported on $E^{+}$, we have

$$
\lim _{k \rightarrow \infty} \mu\left(E_{k, m_{k}}\right)=1
$$

Set $\eta_{k}:=\left.\mu\left(E_{k, m_{k}}\right)^{-1} \mu\right|_{E_{k, m_{k}}}$ for all $k \geq k_{0}$. From Lemma 3.5, we obtain

$$
\lim _{k \rightarrow \infty} \eta_{k}=\mu \quad \text { and } \quad \lim _{k \rightarrow \infty} I_{S}\left(\eta_{k}\right)=I_{S}(\mu) .
$$

Let $k \geq k_{0}$. Replacing in Lemma 3.7 the sets $E$ and $E_{0}$ by $E^{+}$and $E_{k, m_{k}}$, respectively, implies the existence of a sequence $\left(F_{k, i}\right)_{i \in \mathbb{N}}$ of Borel subsets of $E^{+}$ such that

$$
\left.\lim _{i \rightarrow \infty} \mathcal{L}\left(F_{k, i}\right)^{-1} \mathcal{L}\right|_{F_{k, i}}=\eta_{k} \quad \text { and } \quad \lim _{i \rightarrow \infty} I_{s}\left(\left.\mathcal{L}\left(F_{k, i}\right)^{-1} \mathcal{L}\right|_{F_{k, i}}\right)=I_{S}\left(\eta_{k}\right)
$$


Combining this with (3.21), we see that there exists a sequence $\left(i_{k}\right)_{k \in \mathbb{N}}$ of natural numbers such that

$$
\left.\lim _{k \rightarrow \infty} \mathcal{L}\left(F_{k, i_{k}}\right)^{-1} \mathcal{L}\right|_{F_{k, i_{k}}}=\mu \quad \text { and } \quad \lim _{k \rightarrow \infty} I_{S}\left(\left.\mathcal{L}\left(F_{k, i_{k}}\right)^{-1} \mathcal{L}\right|_{F_{k, i_{k}}}\right)=I_{S}(\mu) .
$$

This completes the proof of Proposition 3.8.

The next lemma states that every Lebesgue measurable set with positive Lebesgue density is contained in a Borel set with positive Lebesgue density having the same Hausdorff content as the original set.

Lemma 3.9. Let $R>0$ and $s>0$. Assume that $E \subset B(0, R)$ is a Lebesgue measurable subset of $\mathbb{R}^{d}$. Then there exists a Borel set $X \subset B(0, R)$ such that $E \subset X, \mathcal{L}(X \backslash E)=0$ and $\mathcal{H}_{\infty}^{s}(E)=\mathcal{H}_{\infty}^{s}(X)$. Furthermore, under the additional assumption $E^{+}=E$, we may choose $X$ so that $X^{+}=X$.

ProOF. The definition of $\mathcal{H}_{\infty}^{s}(\cdot)$ implies that, for every $n \in \mathbb{N}$, there exists a sequence $\left(F_{n, i}\right)_{i \in \mathbb{N}}$ of Borel sets satisfying $E \subset \bigcup_{i=1}^{\infty} F_{n, i}$ and $\sum_{i=1}^{\infty}\left(\operatorname{diam} F_{n, i}\right)^{s}<$ $\mathcal{H}_{\infty}^{s}(E)+\frac{1}{n}$. Defining $F:=\bigcap_{n=1}^{\infty} \bigcup_{i=1}^{\infty} F_{n, i}$, it is clear that $F$ is Borel measurable, $E \subset F$ and $\mathcal{H}_{\infty}^{s}(F)=\mathcal{H}_{\infty}^{s}(E)$. Moreover, there exists a Borel set $A \subset B(0, R)$ such that $E \subset A$ and $\mathcal{L}(A)=\mathcal{L}(E)$. Setting $X:=F \cap A$, it is easy to see that $X$ fulfils all the desired properties.

If $E^{+}=E$, the above construction may lead to the situation where $X^{+} \neq X$. However, we have $E \subset X^{+} \subset X$ and, therefore, $\mathcal{H}_{\infty}^{s}\left(X^{+}\right)=\mathcal{H}_{\infty}^{s}(E)=\mathcal{H}_{\infty}^{s}(X)$ and $\mathcal{L}\left(X^{+}\right)=\mathcal{L}(E)=\mathcal{L}(X)$. Note that $\left(X^{+}\right)^{+}=X^{+}$. [Indeed, $\left(A^{+}\right)^{+}=A^{+}$for all Lebesgue measurable sets $A \subset \mathbb{R}^{d}$ since $\mathcal{L}\left(A^{+}\right)=\mathcal{L}(A)$.] Since $X$ is a Borel set, so is $X^{+}$. We complete the proof by deducing that the set $Y:=X^{+}$has the following properties: $Y \subset B(0, R)$ is a Borel set, $Y^{+}=Y, E \subset Y, \mathcal{L}(Y \backslash E)=0$ and $\mathcal{H}_{\infty}^{s}(Y)=\mathcal{H}_{\infty}^{s}(E)$.

The next lemma may be regarded as a complementary result to Lemma 3.2.

LEMMA 3.10. Let $0<t<s<d$ and $R>0$. Then there exists a positive constant $C=C(s, t, d, R)$ such that, for all Lebesgue measurable sets $E \subset B(0, R)$ with $E^{+}=E$, we have

$$
\mathcal{H}_{\infty}^{s}(E) \leq C G_{t}(E)
$$

Proof. We may assume that $E \neq \varnothing$. Since $E^{+}=E \neq \varnothing$, we have $\mathcal{L}(E)>0$ and, therefore, $\mathcal{H}_{\infty}^{s}(E)>0$.

We first assume that $E$ is Borel measurable. By Frostman's lemma [45], Theorem 8.8, there exists a Radon measure $\mu$ supported on $E$ such that $\mu(B(x, r)) \leq r^{s}$ for all $x \in \mathbb{R}^{d}$ and $r>0$ and, moreover, $\mu(E)=c \mathcal{H}_{\infty}^{s}(E)$, where $c$ is a constant 
depending only on $d$. Next, we make a standard calculation in a slightly complicated looking fashion since that will be useful for later purposes (see Section 8). Let $h(r):=r^{t}$ and $\tilde{h}(r):=r^{s}$ for all $r \geq 0$. Set $\delta:=\frac{s}{t}-1$ and $a:=\mu(E)^{-\frac{1}{1+\delta}}$. Then $\tilde{h}(r) \leq h(r)^{1+\delta}$ for all $r>0$. By [45], Theorem 1.15, we have for some constant $c_{1}$ depending on $t$ and $s$ that

$$
\begin{aligned}
0 & <I_{t}(\mu)=\iint \mu\left(\left\{y \in \mathbb{R}^{d}: \frac{1}{h(|x-y|)} \geq u\right\}\right) d u d \mu(x) \\
& \leq \iint \min \left\{\mu(E), \mu\left(B\left(x, h^{-1}\left(u^{-1}\right)\right)\right)\right\} d u d \mu(x) \\
& \leq \int\left(\int_{0}^{a} \mu(E) d u+\int_{a}^{\infty} \tilde{h}\left(h^{-1}\left(u^{-1}\right)\right) d u\right) d \mu(x) \\
& \leq \int\left(\mu(E)^{1-\frac{1}{1+\delta}}+\int_{a}^{\infty} u^{-1-\delta} d u\right) d \mu(x) \\
& \leq c_{1} \mu(E)^{1+\frac{\delta}{1+\delta}} \leq c_{1}\left(c \mathcal{H}_{\infty}^{s}(B(0, R))\right)^{1-\frac{t}{s}} \mu(E) .
\end{aligned}
$$

Thus $0<I_{t}(\mu) \leq \tilde{c} \mu(E)<\infty$, where $\tilde{c}$ is a constant depending only on $t, s$ and $R$.

Applying Proposition 3.8 to $E$, we find a sequence $\left(\mu_{k}\right)_{k \in \mathbb{N}}$ of measures such that $\mu_{k}=\left.\mu(E) \mathcal{L}\left(E_{k}\right)^{-1} \mathcal{L}\right|_{E_{k}}$ and $\lim _{k \rightarrow \infty} I_{t}\left(\mu_{k}\right)=I_{t}(\mu)$. Here, each $E_{k}$ is a Borel measurable subset of $E$ with $0<\mathcal{L}\left(E_{k}\right)<\infty$. For all sufficiently large $k \in \mathbb{N}$, we obtain

$$
\frac{\mu(E)^{2}}{\mathcal{L}\left(E_{k}\right)^{2}} I_{t}\left(E_{k}\right)=I_{t}\left(\mu_{k}\right) \leq 2 I_{t}(\mu) \leq 2 \tilde{c} \mu(E),
$$

giving

$$
\mathcal{H}_{\infty}^{s}(E)=c^{-1} \mu(E) \leq 2 \tilde{c} c^{-1} \frac{\mathcal{L}\left(E_{k}\right)^{2}}{I_{t}\left(E_{k}\right)} \leq 2 \tilde{c} c^{-1} G_{t}(E)
$$

by (1.9). Choosing $C:=2 \tilde{c} c^{-1}$ completes the proof for Borel sets $E$.

The general case of $E$ being Lebesgue measurable may be reduced to the above setting in the following manner. By Lemma 3.9, there exists a Borel set $X \subset B(0, R)$ so that $X^{+}=X, E \subset X, \mathcal{H}_{\infty}^{s}(X)=\mathcal{H}_{\infty}^{s}(E)$ and $\mathcal{L}(X \backslash E)=0$. Since $E \subset X$ and $\mathcal{L}(X \backslash E)=0$, we have $G_{t}(X)=G_{t}(E)$. The above established inequality $\mathcal{H}_{\infty}^{s}(X) \leq C G_{t}(X)$ implies that $\mathcal{H}_{\infty}^{s}(E) \leq C G_{t}(E)$.

Now we are ready to prove Theorem 1.1(c).

Proof of Theorem 1.1(C). Choose $R>0$ such that $\Delta \subset B(0, R)$. Recalling (1.7) and (1.8), the statement follows directly from Lemmas 3.2 and 3.10. 
4. Minimal regular energy. In this section, we introduce a new concept of minimal regular energy and study basic properties of it. We also explain how it can be used to estimate dimensions of random covering sets. The main results are Proposition 4.5 and Lemma 4.7, which are needed in our proof of Theorem 1.1(b).

For $E \subset \mathbb{R}^{d}$, set

$\mathcal{P}_{0}(E):=\left\{\mu \in \mathcal{P}(E): \mu=\left.\sum_{i=1}^{k} c_{i} \mathcal{L}\right|_{E_{i}}, k \in \mathbb{N}, c_{i}>0\right.$ and $E_{i} \subset E$ are Borel sets $\}$.

Recall from Section 3 that $\mathcal{P}(E)$ is the space of Borel probability measures supported on $E$. For $E \subset \mathbb{R}^{d}$ and $0<s<d$, define

$$
\Gamma_{s}(E):= \begin{cases}\inf \left\{I_{s}(\mu): \mu \in \mathcal{P}_{0}(E)\right\} & \text { if } \mathcal{L}(E)>0 \\ \infty & \text { if } \mathcal{L}(E)=0\end{cases}
$$

The quantity $\Gamma_{S}(E)$ is called the minimal regular s-energy of $E$.

LEMMA 4.1. Let $E \in \mathcal{B}\left(\mathbb{R}^{d}\right)$ and $0<s<d$. Then the following properties hold:

(i) If $F \subset E$ is a Borel set, then $\Gamma_{S}(E) \leq \Gamma_{S}(F)$.

(ii) If $\mathcal{L}(E)>0$, then $\Gamma_{s}(E)<\infty$.

(iii) If $E$ is bounded, then $\Gamma_{s}(E)>0$.

(iv) For every $\varepsilon>0$, there exists $\delta=\delta(E, \varepsilon)>0$ such that

$$
\Gamma_{S}(F) \leq \Gamma_{s}(E)+\varepsilon
$$

provided that $F \in \mathcal{B}\left(\mathbb{R}^{d}\right)$ and $\mathcal{L}(E \backslash F)<\delta$.

(v) Let $\left(E_{n}\right)_{n \in \mathbb{N}}$ be a sequence of Borel subsets of $E$. Supposing that $\mathcal{L}(E)<$ $\infty$, we have

$$
\Gamma_{s}\left(\bigcup_{i=1}^{\infty} E_{i}\right)=\lim _{n \rightarrow \infty} \Gamma_{s}\left(\bigcup_{i=1}^{n} E_{i}\right)=\inf _{n \in \mathbb{N}} \Gamma_{s}\left(\bigcup_{i=1}^{n} E_{i}\right)
$$

Proof. Statement (i) is obvious. To verify (ii), choose a compact set $F \subset E$ with $\mathcal{L}(F)>0$ (recall that $\mathcal{L}$ is inner regular), and set $\mu:=\left.\mathcal{L}(F)^{-1} \mathcal{L}\right|_{F}$. Clearly, $\mu \in \mathcal{P}_{0}(E)$. Since $0<s<d$, we have

$$
\begin{aligned}
I_{S}(\mu) & =\mathcal{L}(F)^{-2} \iint_{F \times F}|x-y|^{-s} d \mathcal{L}(x) d \mathcal{L}(y) \\
& \leq \mathcal{L}(F)^{-2} \iint_{B(0, R) \times B(0, R)}|x-y|^{-s} d \mathcal{L}(x) d \mathcal{L}(y)<\infty,
\end{aligned}
$$

where $R>0$ is sufficiently large so that $F \subset B(0, R)$. Hence $\Gamma_{s}(E)<\infty$.

For the purpose of proving (iii), suppose on the contrary that $\Gamma_{S}(E)=0$. Then there exists a sequence $\left(\mu_{n}\right)_{n \in \mathbb{N}}$ such that $\mu_{n} \in \mathcal{P}_{0}(E)$ and $\lim _{n \rightarrow \infty} I_{s}\left(\mu_{n}\right)=0$. 
Since $E$ is bounded, the sequence $\left(\mu_{n}\right)_{n \in \mathbb{N}}$ has at least one accumulation point, say $\mu$, in the weak-star topology. By Lemma 3.4, $I_{S}(\cdot)$ is lower semicontinuous and, therefore, $I_{S}(\mu)=0$, leading to a contradiction since $\mu$ is a Borel probability measure. This completes the proof of (iii).

Next, we verify (iv). We may assume that $\mathcal{L}(E)>0$. Then $\Gamma_{s}(E)<\infty$ by (ii). Let $\varepsilon>0$. By the definition of $\Gamma_{s}(\cdot)$, there exists $\mu=\left.\sum_{i=1}^{k} c_{i} \mathcal{L}\right|_{E_{i}} \in \mathcal{P}_{0}(E)$ such that $I_{S}(\mu) \leq \Gamma_{S}(E)+\frac{\varepsilon}{2}$, where $E_{i} \subset E$ and $\mathcal{L}\left(E_{i}\right)>0$ for all $i=1, \ldots, k$. Define $\delta_{1}:=\min \left\{\mathcal{L}\left(E_{i}\right): i=1, \ldots, k\right\}$,

$$
\gamma:=\sqrt{\frac{\Gamma_{s}(E)+\frac{\varepsilon}{2}}{\Gamma_{s}(E)+\varepsilon}} \quad \text { and } \quad \delta:=(1-\gamma) \delta_{1} .
$$

Let $F \in \mathcal{B}\left(\mathbb{R}^{d}\right)$ with $\mathcal{L}(E \backslash F)<\delta$. We proceed by showing that $\Gamma_{S}(F) \leq \Gamma_{s}(E)+$ $\varepsilon$. First, notice that, for all $i=1, \ldots, k$,

$$
\begin{aligned}
\mathcal{L}\left(E_{i} \cap F\right) & \geq \mathcal{L}\left(E_{i} \cap E\right)-\mathcal{L}(E \backslash F)=\mathcal{L}\left(E_{i}\right)-\mathcal{L}(E \backslash F) \\
& \geq \mathcal{L}\left(E_{i}\right)-\delta \geq \gamma \mathcal{L}\left(E_{i}\right) .
\end{aligned}
$$

Letting $0<\varrho<1$, by the inner regularity of $\mathcal{L}$, there is a compact set $\widetilde{E}_{i} \subset E_{i} \cap F$ such that $\mathcal{L}\left(\widetilde{E}_{i}\right) \geq \varrho \mathcal{L}\left(E_{i} \cap F\right)$ for all $i=1, \ldots, k$. Setting

$$
\mu_{F}:=\left.\frac{1}{c_{F}} \sum_{i=1}^{k} c_{i} \mathcal{L}\right|_{\widetilde{E}_{i}}
$$

where $c_{F}:=\sum_{i=1}^{k} c_{i} \mathcal{L}\left(\widetilde{E}_{i}\right)>0$, the measure $\mu_{F}$ is supported on $F$ and, therefore, $\mu_{F} \in \mathcal{P}_{0}(F)$. Using the fact that $E_{i} \subset E$ for all $i=1, \ldots, k$, we deduce that

$$
\mathcal{L}\left(\widetilde{E}_{i}\right) \geq \varrho \mathcal{L}\left(E_{i} \cap F\right)>\varrho \gamma \mathcal{L}\left(E_{i}\right) .
$$

Thus $c_{F} \geq \sum_{i=1}^{k} c_{i} \varrho \gamma \mathcal{L}\left(E_{i}\right)=\varrho \gamma$ and

$$
I_{S}\left(\mu_{F}\right) \leq\left(c_{F}\right)^{-2} I_{S}(\mu) \leq \rho^{-2} \gamma^{-2}\left(\Gamma_{S}(E)+\frac{\varepsilon}{2}\right)=\varrho^{-2}\left(\Gamma_{S}(E)+\varepsilon\right) .
$$

Hence $\Gamma_{S}(F) \leq \varrho^{-2}\left(\Gamma_{S}(E)+\varepsilon\right)$. Letting $\varrho$ tend to 1 , gives $\Gamma_{S}(F) \leq \Gamma_{S}(E)+\varepsilon$. This completes the proof of (iv).

Finally, (v) follows from (i), (iv) and the fact that

$$
\lim _{n \rightarrow \infty} \mathcal{L}\left(\bigcup_{i=1}^{\infty} E_{i} \backslash\left(\bigcup_{j=1}^{n} E_{j}\right)\right)=0
$$

We proceed by giving an equivalent definition of $\Gamma_{s}(A)$ although we will not apply it in this paper. We use the notation $\mu \ll v$ to indicate that the measure $\mu$ is absolutely continuous with respect to the measure $v$. 
LEMMA 4.2. Let $E \subset \mathbb{R}^{d}$. With convention inf $\varnothing=\infty$, we have

$$
\Gamma_{s}(E)=\inf \left\{I_{s}(\mu): \mu \in \mathcal{P}(E) \text { with } \mu \ll \mathcal{L}\right\} .
$$

PROOF. It is sufficient to show that, for every $\mu \in \mathcal{P}(E)$ with $\mu \ll \mathcal{L}$ and for every $\varepsilon>0$, there exists $\eta \in \mathcal{P}_{0}(E)$ such that

$$
I_{S}(\eta) \leq I_{S}(\mu)+\varepsilon .
$$

To prove the above fact, we denote by $h=\frac{d \mu}{d \mathcal{L}}$ the Radon-Nikodym derivative of $\mu$ with respect to $\mathcal{L}$. Approximating $h$ by step functions, we see that, for every $\delta>0$, there exists a step function $g=\sum_{i=1}^{k} a_{i} \chi_{E_{i}}$, where $a_{i}>0, E_{i}$ is a Borel set and $\bar{E}_{i} \subset E$ for all $i=1, \ldots, k$, so that

$$
\left|I_{S}(\mu)-\iint\right| x-\left.y\right|^{-s} g(x) g(y) d \mathcal{L}(x) d \mathcal{L}(y) \mid<\frac{\varepsilon}{2}
$$

and

$$
\left|\int h(x) d \mathcal{L}(x)-\int g(x) d \mathcal{L}(x)\right|<\delta .
$$

Let $u:=\sum_{i=1}^{k} a_{i} \mathcal{L}\left(E_{i}\right)$. Then $u>1-\delta$ by (4.2). Defining $\eta:=\left.\frac{1}{u} \sum_{i=1}^{k} a_{i} \mathcal{L}\right|_{E_{i}}$ implies that $\eta \in \mathcal{P}_{0}(E)$. Using (4.1), we get for a small enough $\delta$ that

$$
\begin{aligned}
I_{S}(\eta) & =u^{-2} \iint|x-y|^{-s} g(x) g(y) d \mathcal{L}(x) d \mathcal{L}(y) \\
& \leq(1-\delta)^{-2}\left(I_{S}(\mu)+\frac{\varepsilon}{2}\right) \\
& \leq I_{S}(\mu)+\varepsilon .
\end{aligned}
$$

This completes the proof of the lemma.

LEMMA 4.3. Let $\left(E_{n}\right)_{n \in \mathbb{N}}$ be a decreasing sequence of compact subsets of $\mathbb{R}^{d}$, and let $0<s<d$. Assume that there exists $c>0$ such that $\Gamma_{s}\left(E_{n}\right)<c$ for all $n \in \mathbb{N}$. Then $\mathcal{H}_{\infty}^{s}\left(\bigcap_{n=1}^{\infty} E_{n}\right) \geq c^{-1}$. In particular, $\operatorname{dim}_{\mathrm{H}}\left(\bigcap_{n=1}^{\infty} E_{n}\right) \geq s$.

PROOF. According to the definition of $\Gamma_{s}(\cdot)$, for every $n \in \mathbb{N}$, there exists $\mu_{n} \in \mathcal{P}_{0}\left(E_{n}\right)$ so that $I_{s}\left(\mu_{n}\right)<c$. Let $\mu$ be an accumulation point of the sequence $\left(\mu_{n}\right)_{n \in \mathbb{N}}$ in the weak-star topology. Then $\mu$ is supported on $\bigcap_{n=1}^{\infty} E_{n}$ and, furthermore, $I_{S}(\mu) \leq c$ by lower semicontinuity of $I_{S}(\cdot)$ (see Lemma 3.4). The conclusion follows from Remark 3.3.

In the remaining part of this section, we assume that $U \subset \mathbb{R}^{d}$ is open and $\left(A_{n}(x)\right)_{n \in \mathbb{N}}$ is a sequence of $\mathcal{B}\left(\mathbb{R}^{d}\right)$-valued functions defined on $U$ such that:

(C-1) $\mathcal{L}\left(A_{n}(x)\right)<\infty$ for all $x \in U$ and $n \in \mathbb{N}$, and 
$(\mathrm{C}-2) \lim _{y \rightarrow x} \mathcal{L}\left(\left(A_{n}(x) \backslash A_{n}(y)\right) \cup\left(A_{n}(y) \backslash A_{n}(x)\right)\right)=0$ for all $x \in U$ and $n \in \mathbb{N}$.

Let $U^{\mathbb{N}}:=\prod_{n=1}^{\infty} U$ be endowed with the product topology. Consider $\eta \in \mathcal{P}(U)$ and set $\mathbb{P}:=\prod_{i=1}^{\infty} \eta$.

LEMMA 4.4. Let $E \in \mathcal{B}\left(\mathbb{R}^{d}\right)$ with $\mathcal{L}(E)<\infty$. Then, for all $n \in \mathbb{N}$, the mapping

$$
\left(x_{i}\right)_{i=1}^{n} \mapsto \Gamma_{s}\left(E \cap \bigcup_{i=1}^{n} A_{i}\left(x_{i}\right)\right)
$$

is upper semicontinuous on $U^{n}:=\prod_{i=1}^{n} U$. Moreover, the mapping

$$
\mathbf{x}:=\left(x_{i}\right)_{i=1}^{\infty} \mapsto \Gamma_{s}\left(E \cap \bigcup_{i=1}^{\infty} A_{i}\left(x_{i}\right)\right)
$$

is Borel measurable on $U^{\mathbb{N}}$.

Proof. Let $\mathbf{x} \in U^{\mathbb{N}}$ and $n \in \mathbb{N}$. By $(\mathrm{C}-2), \mathcal{L}\left(\bigcup_{i=1}^{n} A_{i}\left(x_{i}\right) \backslash \bigcup_{j=1}^{n} A_{j}\left(y_{j}\right)\right)$ is close to 0 when $\left(y_{i}\right)_{i=1}^{n} \in U^{n}$ is close to $\left(x_{i}\right)_{i=1}^{n}$. Applying Lemma 4.1(iv), we obtain upper semicontinuity (and hence Borel measurability) of the mapping $\left(x_{i}\right)_{i=1}^{n} \mapsto \Gamma_{s}\left(E \cap \bigcup_{i=1}^{n} A_{i}\left(x_{i}\right)\right)$ defined on $U^{n}$ and that of the mapping $\mathbf{x} \mapsto \Gamma_{s}\left(E \cap \bigcup_{i=1}^{n} A_{i}\left(x_{i}\right)\right)$ defined on $U^{\mathbb{N}}$. It follows from Lemma 4.1(v) that

$$
\lim _{n \rightarrow \infty} \Gamma_{s}\left(E \cap \bigcup_{i=1}^{n} A_{i}\left(x_{i}\right)\right)=\Gamma_{s}\left(E \cap \bigcup_{i=1}^{\infty} A_{i}\left(x_{i}\right)\right),
$$

which, in turn, implies Borel measurability of the map $\mathbf{x} \mapsto \Gamma_{S}\left(E \cap \bigcup_{i=1}^{\infty} A_{i}\left(x_{i}\right)\right)$ on $U^{\mathbb{N}}$.

The next proposition provides a sufficient condition for determining a lower bound for Hausdorff dimensions of typical random covering sets.

Proposition 4.5. Let $E \subset \mathbb{R}^{d}$ be compact with $\mathcal{L}(E)>0$. In addition to $(\mathrm{C}-1)$ and $(\mathrm{C}-2)$, assume that $A_{n}(x)$ is compact for all $x \in U$ and $n \in \mathbb{N}$. Let $0<s<d$. Suppose that, for all compact sets $F \subset E$, we have for $\mathbb{P}$-almost all $\mathbf{x} \in U^{\mathbb{N}}$ that

$$
\Gamma_{s}\left(F \cap\left(\bigcup_{i=n}^{\infty} A_{i}\left(x_{i}\right)\right)\right)=\Gamma_{s}(F) \quad \text { for all } n \in \mathbb{N}
$$

Then

$$
\mathcal{H}_{\infty}^{s}\left(\limsup _{n \rightarrow \infty} A_{n}\left(x_{n}\right)\right) \geq \Gamma_{s}(E)^{-1} \quad \text { and } \quad \operatorname{dim}_{\mathrm{H}}\left(\limsup _{n \rightarrow \infty} A_{n}\left(x_{n}\right)\right) \geq s
$$

for $\mathbb{P}$-almost all $\mathbf{x} \in U^{\mathbb{N}}$. 
PROOF. From (4.3), we obtain

$$
\Gamma_{s}\left(E \cap\left(\bigcup_{i=1}^{\infty} A_{i}\left(x_{i}\right)\right)\right)=\Gamma_{s}(E)
$$

for $\mathbb{P}$-almost all $\mathbf{x} \in U^{\mathbb{N}}$. Note that $0<\Gamma_{S}(E)<\infty$ by Lemma 4.1 claims (ii) and (iii). Letting $\ell>2$, Lemma 4.1(v) and Lemma 4.4 imply the existence of a Borel measurable function $n_{1}: U^{\mathbb{N}} \rightarrow \mathbb{N}$ such that

$$
\Gamma_{s}\left(E \cap\left(\bigcup_{i=1}^{n_{1}(\mathbf{x})} A_{i}\left(x_{i}\right)\right)\right)<\left(1+\ell^{-1}\right) \Gamma_{s}(E)
$$

for $\mathbb{P}$-almost all $\mathbf{x} \in U^{\mathbb{N}}$. By Lemma 4.1(i), we find $N_{1} \in \mathbb{N}$ and a Borel set $\Lambda_{1} \subset$ $U^{N_{1}}$ so that

$$
\eta^{N_{1}}\left(\Lambda_{1}\right)>1-\ell^{-1} \quad \text { and } \quad \Gamma_{s}\left(E \cap\left(\bigcup_{i=1}^{N_{1}} A_{i}\left(x_{i}\right)\right)\right)<\left(1+\ell^{-1}\right) \Gamma_{s}(E)
$$

for all $\left(x_{1}, \ldots, x_{N_{1}}\right) \in \Lambda_{1}$, where $\eta^{N_{1}}:=\prod_{i=1}^{N_{1}} \eta$. Applying (4.3) with $F=E \cap$ $\left(\bigcup_{i=1}^{N_{1}} A_{i}\left(x_{i}\right)\right)$, gives for all $\left(x_{1}, \ldots, x_{N_{1}}\right) \in \Lambda_{1}$ that

$$
\Gamma_{s}\left(E \cap\left(\bigcup_{i=1}^{N_{1}} A_{i}\left(x_{i}\right)\right) \cap\left(\bigcup_{j=N_{1}+1}^{\infty} A_{j}\left(x_{j}\right)\right)\right)<\left(1+\ell^{-1}\right) \Gamma_{s}(E)
$$

for $\left(\prod_{i=N_{1}+1}^{\infty} \eta\right)$-almost all $\left(x_{N_{1}+1}, x_{N_{1}+2}, \ldots\right) \in \prod_{i=N_{1}+1}^{\infty} U$. Moreover, by Fubini's theorem, inequality (4.5) holds for $\mathbb{P}$-almost all $\mathbf{x} \in \Lambda_{1} \times \prod_{i=N_{1}+1}^{\infty} U$. As above, it follows from Lemma 4.1(i) that there exist a natural number $N_{2}>N_{1}$ and a Borel set $\Lambda_{2} \subset \Lambda_{1} \times \prod_{i=N_{1}+1}^{N_{2}} U \subset U^{N_{2}}$ with $\eta^{N_{2}}\left(\Lambda_{2}\right) \geq \eta^{N_{1}}\left(\Lambda_{1}\right)-\ell^{-2}$ such that

$$
\Gamma_{s}\left(E \cap\left(\bigcup_{i=1}^{N_{1}} A_{i}\left(x_{i}\right)\right) \cap\left(\bigcup_{j=N_{1}+1}^{N_{2}} A_{j}\left(x_{j}\right)\right)\right)<\left(1+\ell^{-1}\right)\left(1+\ell^{-2}\right) \Gamma_{s}(E)
$$

for all $\left(x_{1}, \ldots, x_{N_{2}}\right) \in \Lambda_{2}$.

By induction, we deduce that there exist a strictly increasing sequence $\left(N_{n}\right)_{n \in \mathbb{N}}$ of positive integers and a sequence $\left(\Lambda_{n}\right)_{n \in \mathbb{N}}$ of Borel sets such that $\Lambda_{n} \subset U^{N_{n}}$, $\Lambda_{n+1} \subset \Lambda_{n} \times \prod_{i=N_{n}+1}^{N_{n+1}} U$,

$$
\eta^{N_{n+1}}\left(\Lambda_{n+1}\right) \geq \eta^{N_{n}}\left(\Lambda_{n}\right)-\ell^{-n-1}
$$

and

$$
\Gamma_{s}\left(E \cap \bigcap_{k=1}^{n}\left(\bigcup_{i=N_{k-1}+1}^{N_{k}} A_{i}\left(x_{i}\right)\right)\right)<\left(\prod_{i=1}^{n}\left(1+\ell^{-i}\right)\right) \Gamma_{s}(E)
$$


for all $\left(x_{1}, \ldots, x_{N_{n}}\right) \in \Lambda_{n}$. Here $N_{0}:=0$. Defining $\Omega:=\bigcap_{n=1}^{\infty}\left(\Lambda_{n} \times \prod_{i=N_{1}+1}^{\infty} U\right)$ and using (4.6), implies that

$$
\mathbb{P}(\Omega) \geq 1-\sum_{n=1}^{\infty} \ell^{-n}=\frac{\ell-2}{\ell-1} .
$$

Moreover, by (4.7) and Lemma 4.3, we have

$$
\begin{aligned}
& \mathcal{H}_{\infty}^{s}\left(\bigcap_{n=1}^{\infty} \bigcup_{i=N_{n-1}+1}^{N_{n}} A_{i}\left(x_{i}\right)\right) \geq\left(\left(\prod_{i=1}^{\infty}\left(1+\ell^{-i}\right)\right) \Gamma_{s}(E)\right)^{-1} \text { and } \\
& \operatorname{dim}_{H}\left(\bigcap_{n=1}^{\infty} \bigcup_{i=N_{n-1}+1}^{N_{n}} A_{i}\left(x_{i}\right)\right) \geq s
\end{aligned}
$$

for all $\mathbf{x} \in \Omega$. This gives $\operatorname{dim}_{H}\left(\lim \sup _{n \rightarrow \infty} A_{n}\left(x_{n}\right)\right) \geq s$ for all $\mathbf{x} \in \Omega$. Since $\ell$ can be taken arbitrarily large, it follows from (4.8) that

$$
\mathcal{H}_{\infty}^{s}\left(\limsup _{n \rightarrow \infty} A_{n}\left(x_{n}\right)\right) \geq \Gamma_{s}(E)^{-1} \quad \text { and } \quad \operatorname{dim}_{\mathrm{H}}\left(\limsup _{n \rightarrow \infty} A_{n}\left(x_{n}\right)\right) \geq s
$$

for $\mathbb{P}$-almost all $\mathbf{x} \in U^{\mathbb{N}}$.

The above proof readily leads to the following deterministic version of Proposition 4.5 , which may be of independent interest.

Proposition 4.6. Let $E \subset \mathbb{R}^{d}$ be compact with $\mathcal{L}(E)>0$, and let $\left(A_{n}\right)_{n \in \mathbb{N}}$ be a sequence of compact subsets of $\mathbb{R}^{d}$. Let $0<s<d$. Suppose that, for all compact sets $F \subset E$, we have that

$$
\Gamma_{s}\left(F \cap\left(\bigcup_{i=n}^{\infty} A_{i}\right)\right)=\Gamma_{s}(F) \quad \text { for all } n \in \mathbb{N} .
$$

Then

$$
\mathcal{H}_{\infty}^{s}\left(\limsup _{n \rightarrow \infty} A_{n}\right) \geq \Gamma_{s}(E)^{-1} \quad \text { and } \quad \operatorname{dim}_{\mathrm{H}}\left(\limsup _{n \rightarrow \infty} A_{n}\right) \geq s .
$$

In the last result of this section, we give a sufficient condition for the validity of (4.3). Recall that, by the definition of $\Gamma_{s}(\cdot)$, the inequality (4.3) is valid for all $F \in \mathcal{B}\left(\mathbb{R}^{d}\right)$ with $\mathcal{L}(F)=0$.

LEMMA 4.7. Let $F \in \mathcal{B}\left(\mathbb{R}^{d}\right)$ with $\mathcal{L}(F)>0$, and let $0<s<d$. Assume that, for every $\varepsilon>0$ and $\delta>0$ and for every $n \in \mathbb{N}$, there exist an integer $N>n$ and $a$ Borel measurable set $\Omega \subset U^{\mathbb{N}}$ with $\mathbb{P}(\Omega)>1-\delta$ such that

$$
\int_{\Omega} \Gamma_{s}\left(F \cap \bigcup_{i=n}^{N} A_{i}\left(x_{i}\right)\right) d \mathbb{P}(\mathbf{x})<\Gamma_{s}(F)+\varepsilon .
$$


Then, for $\mathbb{P}$-almost all $\mathbf{x} \in U^{\mathbb{N}}$,

$$
\Gamma_{s}\left(F \cap \bigcup_{i=n}^{\infty} A_{i}\left(x_{i}\right)\right)=\Gamma_{s}(F)
$$

for all $n \in \mathbb{N}$.

Proof. Let $n \in \mathbb{N}$ and $\gamma>0$. By Lemma 4.1(i),

$$
\Gamma_{s}\left(F \cap \bigcup_{i=n}^{N} A_{i}\left(x_{i}\right)\right) \geq \Gamma_{s}\left(F \cap \bigcup_{i=n}^{\infty} A_{i}\left(x_{i}\right)\right) \geq \Gamma_{s}(F)
$$

for all $\mathbf{x} \in U^{\mathbb{N}}$ and $N \in \mathbb{N}$. Let

$$
\Omega^{\prime}:=\left\{x \in U^{\mathbb{N}}: \Gamma_{s}\left(F \cap\left(\bigcup_{i=n}^{\infty} A_{i}\left(x_{i}\right)\right)\right) \geq \Gamma_{s}(F)+\gamma\right\} .
$$

It follows from Lemma 4.4 that $\Omega^{\prime}$ is a Borel set. We show that $\mathbb{P}\left(\Omega^{\prime}\right)=0$. Suppose on the contrary that $\mathbb{P}\left(\Omega^{\prime}\right)>0$ and choose

$$
\varepsilon:=\frac{\mathbb{P}\left(\Omega^{\prime}\right) \gamma}{2} \quad \text { and } \quad \delta:=\frac{\mathbb{P}\left(\Omega^{\prime}\right) \gamma}{2\left(\gamma+2 \Gamma_{s}(F)\right)} .
$$

Recall that $\Gamma_{S}(F)<\infty$ by Lemma 4.1(ii). Then, for all integers $N>n$ and for all Borel measurable sets $\Omega \subset U^{\mathbb{N}}$ with $\mathbb{P}(\Omega)>1-\delta$, we have

$$
\begin{aligned}
\int_{\Omega} \Gamma_{s} & \left(F \cap \bigcup_{i=n}^{N} A_{i}\left(x_{i}\right)\right) d \mathbb{P}(\mathbf{x}) \\
& \geq \int_{\Omega \backslash \Omega^{\prime}} \Gamma_{s}(F) d \mathbb{P}(\mathbf{x})+\int_{\Omega \cap \Omega^{\prime}} \Gamma_{s}(F)+\gamma d \mathbb{P}(\mathbf{x}) \\
& =\mathbb{P}\left(\Omega \backslash \Omega^{\prime}\right) \Gamma_{s}(F)+\mathbb{P}\left(\Omega \cap \Omega^{\prime}\right)\left(\Gamma_{s}(F)+\gamma\right) \\
& \geq\left(\mathbb{P}(\Omega)-\mathbb{P}\left(\Omega^{\prime}\right)\right) \Gamma_{s}(F)+\left(\mathbb{P}\left(\Omega^{\prime}\right)+\mathbb{P}(\Omega)-1\right)\left(\Gamma_{s}(F)+\gamma\right) \\
& =(2 \mathbb{P}(\Omega)-1) \Gamma_{s}(F)+\left(\mathbb{P}\left(\Omega^{\prime}\right)+\mathbb{P}(\Omega)-1\right) \gamma \\
& \geq(1-2 \delta) \Gamma_{S}(F)+\left(\mathbb{P}\left(\Omega^{\prime}\right)-\delta\right) \gamma \quad[\text { by }(4.11)] \\
& =\Gamma_{S}(F)+\varepsilon .
\end{aligned}
$$

This contradicts (4.10) and completes the proof.

5. Lower bound for Hausdorff dimension. The main purpose of this section is to verify Theorem 1.1(b). This is achieved by showing first that, under certain assumptions on the measure $\eta \in \mathcal{P}(U)$ and the sequence $\left(A_{n}(x)\right)_{n \in \mathbb{N}}$, the assumption (4.10) of Lemma 4.7 holds. Theorem 1.1(b) then follows by applying Lemma 4.7 and Proposition 4.5.

We start with a simple observation on independent random variables. 
LEMMA 5.1. Let $\left(a_{n}\right)_{n \in \mathbb{N}}$ be a sequence of positive numbers such that $\sum_{n=1}^{\infty} a_{n}=\infty$, and let $0<c<1$. Suppose that $\left(\omega_{n}\right)_{n \in \mathbb{N}}$ is a sequence of independent random variables with $\omega_{n} \in\{0\} \cup\left[a_{n}, \infty[\right.$ and the probability $\mathbb{P}$ satisfies

$$
\mathbb{P}\left(\omega_{n} \neq 0\right) \geq c .
$$

Then, for all $N \in \mathbb{N}$ and $C \geq 0$, we have

$$
\lim _{M \rightarrow \infty} \mathbb{P}\left(\sum_{n=N}^{M} \omega_{n} \geq C\right)=1 .
$$

ProOF. Observe that the claim is equivalent to the statement

$$
\sum_{n=1}^{\infty} \omega_{n}=\infty \quad \mathbb{P} \text {-almost surely. }
$$

Assuming to the contrary that this is not true, Kolmogorov's zero-one law implies that

$$
\sum_{n=1}^{\infty} \omega_{n}<\infty \quad \mathbb{P} \text {-almost surely. }
$$

Define $b_{n}:=\min \left\{1, a_{n}\right\}$ for $n \in \mathbb{N}$. Then either $b_{n}=1$ for infinitely many $n \in$ $\mathbb{N}$, or $b_{n}=a_{n}$ for all sufficiently large $n \in \mathbb{N}$. In both of these cases, we have $\sum_{n=1}^{\infty} b_{n}=\infty$. Defining

$$
\tilde{\omega}_{n}:= \begin{cases}0 & \text { if } \omega_{n}=0 \\ 1 & \text { if } \omega_{n} \neq 0\end{cases}
$$

gives

$$
\omega_{n} \geq \tilde{\omega}_{n} b_{n}
$$

for all $n \in \mathbb{N}$. In particular, $\sum_{n=1}^{\infty} \tilde{\omega}_{n} b_{n}<\infty \mathbb{P}$-almost surely by (5.2). By Kolmogorov's three series theorem, there exists $\alpha>0$ such that:

(i) $\sum_{n=1}^{\infty} \mathbb{P}\left(\tilde{\omega}_{n} b_{n} \geq \alpha\right)$ converges and

(ii) $\sum_{n=1}^{\infty} \mathbb{E}\left(\tilde{\omega}_{n} b_{n} \chi_{\left\{\tilde{\omega}_{n} b_{n} \leq \alpha\right\}}\right)$ converges.

Assume first that $\limsup _{n \rightarrow \infty} b_{n}=b>0$. By inequality (5.1), the sum in (i) diverges for all $\alpha<b$ and also for $\alpha=b$ provided that $b_{n}>b$ for infinitely many $n \in \mathbb{N}$, while the sum in (ii) diverges for all $\alpha>b$ and also for $\alpha=b$ provided that $b_{n} \leq b$ for all large enough $n \in \mathbb{N}$, since $\mathbb{E}\left(\tilde{\omega}_{n} b_{n} \chi_{\left\{\tilde{\omega}_{n} b_{n} \leq \alpha\right\}}\right) \geq c b_{n}$. This leads to a contradiction. Supposing that $\lim _{n \rightarrow \infty} b_{n}=0$, inequality (5.1) implies the divergence of the sum in (ii) for all $\alpha>0$, which is a contradiction. This completes the proof. 
In the remaining part of this section, let $U \subset \mathbb{R}^{d}$ be open and let $E \subset U$ be a compact set with $\mathcal{L}(E)>0$. Assume that $\eta \in \mathcal{P}(U)$ satisfies $\eta(E)>0,\left.\left.\eta\right|_{E} \ll \mathcal{L}\right|_{E}$ and

$$
\sup _{x, y \in E} \frac{h(x)}{h(y)}<\infty
$$

where $h:=\frac{\left.d \eta\right|_{E}}{d \mathcal{L}}$ is the Radon-Nikodym derivative of $\left.\eta\right|_{E}$ with respect to $\mathcal{L}$. Set $\mathbb{P}:=\prod_{i=1}^{\infty} \eta$. Let $0<s<d$. Next, we define a special sequence $\left(A_{n}(x)\right)_{n \in \mathbb{N}}$.

Definition 5.2. Let $y_{0} \in \mathbb{R}^{d}$. Assume that $\left(K_{n}\right)_{n \in \mathbb{N}}$ is a sequence of compact sets in $\mathbb{R}^{d}$ satisfying:

(i) $\mathcal{L}\left(K_{n}\right)>0$,

(ii) $\lim _{n \rightarrow \infty} \operatorname{diam} K_{n}=0$,

(iii) $\lim _{n \rightarrow \infty} \operatorname{dist}\left(y_{0}, K_{n}\right)=0$ and

(iv) $\sum_{n=1}^{\infty} g_{s}\left(K_{n}\right)=\infty$ [recall (1.4)].

Choose $r_{0}>0$ such that $K_{n} \subset B\left(y_{0}, r_{0}\right)$ for all $n \in \mathbb{N}$. Assume that $W: U \times$ $B\left(y_{0}, r_{0}\right) \rightarrow \mathbb{R}^{d}$ is a uniform bidiffeomorphism (recall Definition 2.1) satisfying $W\left(x, y_{0}\right)=x$ for all $x \in U$. Define $A_{n}(x):=W\left(x, K_{n}\right)$ for all $x \in U$ and $n \in \mathbb{N}$.

The sequence $\left(A_{n}(x)\right)_{n \in \mathbb{N}}$ has the following properties.

Lemma 5.3. Let $\left(A_{n}(x)\right)_{n \in \mathbb{N}}$ be as in Definition 5.2. Then the properties (C-1) and (C-2) from Section 4 are satisfied. Furthermore,

(C-3) for every $\varepsilon>0$ and for every Borel set $F \subset E$ with $\mathcal{L}(F)>0$, there exists $N \in \mathbb{N}$ so that

$$
\mathcal{L}\left(\left\{x \in F: \frac{\mathcal{L}\left(F \cap A_{n}(x)\right)}{\mathcal{L}\left(A_{n}(x)\right)} \geq 1-\varepsilon\right\}\right) \geq(1-\varepsilon) \mathcal{L}(F)
$$

for all $n \geq N$, and

(C-4) for all Borel sets $F_{1}, F_{2} \subset E$ with positive Lebesgue measure and for every $\varepsilon>0$, there exists $N \in \mathbb{N}$ such that

$$
\begin{aligned}
& \iint_{F_{1} \times F_{2}} \iint_{A_{n}(x) \times A_{m}(y)}|u-v|^{-s} d \mathcal{L}(u) d \mathcal{L}(v) d \mathcal{L}(x) d \mathcal{L}(y) \\
& \quad \leq(1+\varepsilon) \iint_{F_{1} \times F_{2}} \mathcal{L}\left(A_{n}(x)\right) \mathcal{L}\left(A_{m}(y)\right)|x-y|^{-s} d \mathcal{L}(x) d \mathcal{L}(y)
\end{aligned}
$$

for all $n, m \geq N$.

Proof. Property (C-1) is clearly valid. Note that $\mathcal{L}(F)=\lim _{\delta \rightarrow 0} \mathcal{L}\left(\bar{V}_{\delta}(F)\right)$ for all compact sets $F \subset \mathbb{R}^{d}$ [recall (2.4)]. Let $n \in \mathbb{N}$. Since $W$ is a uniform bidiffeomorphism, we have, for every $\delta>0$, that $A_{n}(y) \subset \bar{V}_{\delta}\left(A_{n}(x)\right)$ provided 
$y$ is close enough to $x$. Thus $\lim _{y \rightarrow x} \mathcal{L}\left(A_{n}(x) \backslash A_{n}(y)\right)=0$ by the continuity of $x \mapsto \mathcal{L}\left(A_{n}(x)\right)$. Furthermore, for every $\varepsilon>0$ and $x \in U$, there exist $\delta_{1}, \delta_{2}>0$ such that $\mathcal{L}\left(\bar{V}_{\delta_{1}}\left(A_{n}(y)\right) \backslash A_{n}(y)\right)<\varepsilon$ for all $y \in B\left(x, \delta_{2}\right)$. This, in turn, gives $\lim _{y \rightarrow x} \mathcal{L}\left(A_{n}(y) \backslash A_{n}(x)\right)=0$, implying (C-2). Property (C-3) follows from Lemma 2.3 and properties (ii) and (iii) of Definition 5.2. Finally, (C-4) is given by Lemma 2.4 and items (ii) and (iii) of Definition 5.2.

Now we are ready to prove that the assumption (4.10) of Lemma 4.7 is satisfied for compact sets.

Proposition 5.4. Let $F \subset E$ be a compact set with $\mathcal{L}(F)>0$. Then, for every $\varepsilon>0, \delta>0$ and $n \in \mathbb{N}$, there exist an integer $N>n$ and a Borel measurable set $\Omega \subset U^{\mathbb{N}}$ with $\mathbb{P}(\Omega)>1-\delta$ such that

$$
\int_{\Omega} \Gamma_{s}\left(F \cap \bigcup_{i=n}^{N} A_{i}\left(x_{i}\right)\right) d \mathbb{P}(\mathbf{x})<\Gamma_{s}(F)+\varepsilon .
$$

ProOF. Let $\varepsilon>0, \delta>0$ and $n \in \mathbb{N}$. Choose $\mu=\left.\sum_{i=1}^{k} c_{i} \mathcal{L}\right|_{F_{i}} \in \mathcal{P}_{0}(F)$ satisfying

$$
I_{s}(\mu)<\Gamma_{s}(F)+\frac{\varepsilon}{2} .
$$

Let $0<\gamma<1$ be sufficiently small (which will be determined later). By partitioning $F_{i}$ into smaller Borel sets, if necessary, such that each new $F_{i}$ is an approximate level set of the density $h$ with small diameter and with $\eta\left(F_{i}\right)>0$ [recall that $\mathcal{L}(F)>0$ implies $\eta(F)>0$ by (5.4)], we may assume that, for all $i=1, \ldots, k$ and $m \geq n$,

$$
\sup _{x, y \in F_{i}} \max \left\{\frac{h(x)}{h(y)}, \frac{\mathcal{L}\left(A_{m}(x)\right)}{\mathcal{L}\left(A_{m}(y)\right)}, \frac{I_{s}\left(A_{m}(x)\right)}{I_{s}\left(A_{m}(y)\right)}\right\} \leq 1+\gamma .
$$

For every $i=1, \ldots, k$, fix $z_{i} \in F_{i}$. Define, for all $m \geq n$,

$$
F_{i, m}:=\left\{x \in F_{i}: \frac{\mathcal{L}\left(F_{i} \cap A_{m}(x)\right)}{\mathcal{L}\left(A_{m}(x)\right)}>1-\gamma\right\} .
$$

Using the fact that the map $x \mapsto \mathcal{L}\left(F \cap A_{n}(x)\right)$ is Borel measurable (see the proof of Lemma 2.3), we deduce that $F_{i, m}$ is a Borel set. By (C-3) and (C-4), there exists an integer $M>n$ such that

$$
\mathcal{L}\left(F_{i, m}\right) \geq(1-\gamma) \mathcal{L}\left(F_{i}\right)
$$

for all $i=1, \ldots, k$ and $m \geq M$ and, moreover,

$$
\begin{aligned}
& \iint_{F_{i} \times F_{j}} \iint_{A_{m}(x) \times A_{p}(y)}|u-v|^{-s} d \mathcal{L}(u) d \mathcal{L}(v) d \mathcal{L}(x) d \mathcal{L}(y) \\
& \quad \leq(1+\gamma)^{3} \mathcal{L}\left(A_{m}\left(z_{i}\right)\right) \mathcal{L}\left(A_{p}\left(z_{j}\right)\right) \iint_{F_{i} \times F_{j}}|x-y|^{-s} d \mathcal{L}(x) d \mathcal{L}(y)
\end{aligned}
$$


for all $i=1, \ldots, k$ and $m, p \geq M$.

Applying Lemma 5.1 with $a_{m}=g_{s}\left(A_{m}\left(z_{i}\right)\right)$ and $\omega_{m}=\chi_{F_{i, m}} g_{s}\left(A_{m}\left(z_{i}\right)\right)$ [recall (1.4)], Definition 5.2(iv) together with inequalities (2.1) and (5.7) imply that we may choose integers $M_{1}:=M<M_{2}<\cdots<M_{k+1}$ recursively such that

$$
\mathbb{P}\left(\left\{\mathbf{x} \in U^{\mathbb{N}}: \sum_{m=M_{i}}^{M_{i+1}-1} \chi_{F_{i, m}}\left(x_{m}\right) g_{s}\left(A_{m}\left(z_{i}\right)\right) \geq \gamma^{-1}\right\}\right) \geq 1-\frac{\gamma}{k}
$$

for all $i=1, \ldots, k$. Let $N:=M_{k+1}$. Define

$$
\Omega_{i}:=\left\{\mathbf{x} \in U^{\mathbb{N}}: \sum_{m=M_{i}}^{M_{i+1}-1} \chi_{F_{i, m}}\left(x_{m}\right) g_{s}\left(A_{m}\left(z_{i}\right)\right) \geq \gamma^{-1}\right\}
$$

for all $i=1, \ldots, k$ and set

$$
\Omega:=\bigcap_{i=1}^{k} \Omega_{i}
$$

Then $\Omega$ is a Borel set with $\mathbb{P}(\Omega) \geq 1-\gamma$.

For all $\mathbf{x} \in \Omega$, we define a finite Borel measure $\mu^{\mathbf{x}}$ as

$$
\mu^{\mathbf{x}}:=\left.\sum_{i=1}^{k} \sum_{m \in S_{i}(\mathbf{x})} c_{i, m}(\mathbf{x}) \mathcal{L}\right|_{F_{i} \cap A_{m}\left(x_{m}\right)},
$$

where

$$
\begin{aligned}
S_{i}(\mathbf{x}) & :=\left\{m \in \mathbb{N}: M_{i} \leq m<M_{i+1}, x_{m} \in F_{i, m}\right\} \quad \text { and } \\
c_{i, m}(\mathbf{x}) & :=c_{i} \mathcal{L}\left(F_{i}\right) \frac{\mathcal{L}\left(A_{m}\left(z_{i}\right)\right)}{I_{S}\left(A_{m}\left(z_{i}\right)\right)}\left(\sum_{p \in S_{i}(\mathbf{x})} g_{s}\left(A_{p}\left(z_{i}\right)\right)\right)^{-1} .
\end{aligned}
$$

Since $F$ and $A_{i}\left(x_{i}\right)$ are compact the measure $\mu^{\mathbf{x}}$ is supported on $F \cap \bigcup_{i=n}^{N} A_{i}\left(x_{i}\right)$. Notice that, if $x_{m} \in F_{i, m}$, inequality (5.6) results in

$$
\mathcal{L}\left(F_{i} \cap A_{m}\left(x_{m}\right)\right) \geq(1-\gamma) \mathcal{L}\left(A_{m}\left(x_{m}\right)\right) \geq(1-\gamma)(1+\gamma)^{-1} \mathcal{L}\left(A_{m}\left(z_{i}\right)\right),
$$

which, in turn, yields

$$
\begin{aligned}
\left\|\mu^{\mathbf{x}}\right\| & =\sum_{i=1}^{k} c_{i} \mathcal{L}\left(F_{i}\right) \sum_{m \in S_{i}(\mathbf{x})} \frac{\mathcal{L}\left(A_{m}\left(z_{i}\right)\right) \mathcal{L}\left(F_{i} \cap A_{m}\left(x_{m}\right)\right)}{I_{S}\left(A_{m}\left(z_{i}\right)\right)}\left(\sum_{p \in S_{i}(\mathbf{x})} g_{s}\left(A_{p}\left(z_{i}\right)\right)\right)^{-1} \\
& \geq(1-\gamma)(1+\gamma)^{-1} \sum_{i=1}^{k} c_{i} \mathcal{L}\left(F_{i}\right) \sum_{m \in S_{i}(\mathbf{x})} \frac{\mathcal{L}\left(A_{m}\left(z_{i}\right)\right)^{2}}{I_{S}\left(A_{m}\left(z_{i}\right)\right)}\left(\sum_{p \in S_{i}(\mathbf{x})} g_{s}\left(A_{p}\left(z_{i}\right)\right)\right)^{-1} \\
& =(1-\gamma)(1+\gamma)^{-1},
\end{aligned}
$$


where $\left\|\mu^{\mathbf{x}}\right\|$ represents the total mass of $\mu^{\mathbf{x}}$. Since $\left\|\mu^{\mathbf{x}}\right\|^{-1} \mu^{\mathbf{x}} \in \mathcal{P}_{0}(F \cap$ $\left.\bigcup_{i=n}^{N} A_{i}\left(x_{i}\right)\right)$, we have

$$
\Gamma_{s}\left(F \cap \bigcup_{i=n}^{N} A_{i}\left(x_{i}\right)\right) \leq I_{s}\left(\left\|\mu^{\mathbf{x}}\right\|^{-1} \mu^{\mathbf{x}}\right)=\frac{I_{s}\left(\mu^{\mathbf{x}}\right)}{\left\|\mu^{\mathbf{x}}\right\|^{2}} \leq \frac{(1+\gamma)^{2}}{(1-\gamma)^{2}} I_{s}\left(\mu^{\mathbf{x}}\right) .
$$

In what follows, we estimate $\int_{\Omega} I_{S}\left(\mu^{\mathbf{x}}\right) d \mathbb{P}(\mathbf{x})$. Set

$$
\begin{aligned}
\mathcal{S}_{\Omega}:= & \left\{\mathbf{S}=\left(S_{i}\right)_{i=1}^{k}: S_{i} \subset\left\{M_{i}, M_{i}+1, \ldots, M_{i+1}-1\right\}\right. \text { and } \\
& \left.\sum_{p \in S_{i}} g_{s}\left(A_{p}\left(z_{i}\right)\right) \geq \gamma^{-1}\right\} .
\end{aligned}
$$

For $\mathbf{S} \in \mathcal{S}_{\Omega}$, define

$$
\begin{aligned}
\pi^{-1}(\mathbf{S}):= & \bigcap_{i=1}^{k}\left\{\mathbf{x} \in U^{\mathbb{N}}: x_{m} \in F_{i, m} \text { if } m \in S_{i}, \text { and } x_{m} \in\left(F_{i, m}\right)^{c}\right. \\
& \text { if } \left.m \in\left\{M_{i}, M_{i}+1, \ldots, M_{i+1}-1\right\} \backslash S_{i}\right\} .
\end{aligned}
$$

Clearly, $\pi^{-1}(\mathbf{S})$ is a Borel set. Observe that $\Omega=\bigcup_{\mathbf{S} \in \mathcal{S}_{\Omega}} \pi^{-1}(\mathbf{S})$, where the union is disjoint. Let

$$
J_{S}(A, B):=\iint_{A \times B}|x-y|^{-s} d \mathcal{L}(x) d \mathcal{L}(y)
$$

for all Lebesgue measurable sets $A, B \subset \mathbb{R}^{d}$. Consider $\mathbf{S} \in \mathcal{S}_{\Omega}$ and define

$$
Q_{S_{i}}:=\sum_{m \in S_{i}} g_{s}\left(A_{m}\left(z_{i}\right)\right)
$$

for all $i=1, \ldots, k$. Then

$$
\begin{aligned}
\int_{\pi^{-1}(\mathbf{S})} & I_{S}\left(\mu^{\mathbf{x}}\right) d \mathbb{P}(\mathbf{x}) \\
\leq & \sum_{i=1}^{k} \sum_{j=1}^{k} \sum_{m \in S_{i}} \sum_{p \in S_{j}} \frac{c_{i} c_{j} \mathcal{L}\left(F_{i}\right) \mathcal{L}\left(F_{j}\right) \mathcal{L}\left(A_{m}\left(z_{i}\right)\right) \mathcal{L}\left(A_{p}\left(z_{j}\right)\right)}{Q_{S_{i}} Q_{S_{j}} I_{S}\left(A_{m}\left(z_{i}\right)\right) I_{s}\left(A_{p}\left(z_{j}\right)\right)} \\
& \times \int_{\pi^{-1}(\mathbf{S})} J_{S}\left(A_{m}\left(x_{m}\right), A_{p}\left(x_{p}\right)\right) d \mathbb{P}(\mathbf{x}) .
\end{aligned}
$$

In order to complete the proof of Proposition 5.4, we need two more lemmas.

LEMMA 5.5. Let $(Y, \mathcal{F}, v)$ be a probability space, and let $u: Y \times Y \rightarrow \mathbb{R}$ and $\tilde{u}: Y \rightarrow \mathbb{R}$ be nonnegative measurable functions. Let $E_{1}, \ldots, E_{N} \in \mathcal{F}$ with 
$v\left(E_{i}\right)>0$ for all $i=1, \ldots, N$. Then we have

$$
\begin{aligned}
& \int\left(\prod_{i=1}^{N} \chi_{E_{i}}\left(y_{i}\right)\right) u\left(y_{1}, y_{2}\right) \prod_{j=1}^{N} d v\left(y_{j}\right) \\
& \quad=\frac{\prod_{i=1}^{N} v\left(E_{i}\right)}{v\left(E_{1}\right) v\left(E_{2}\right)} \int_{E_{1} \times E_{2}} u\left(y_{1}, y_{2}\right) d v\left(y_{1}\right) d v\left(y_{2}\right)
\end{aligned}
$$

and

$$
\int\left(\prod_{i=1}^{N} \chi_{E_{i}}\left(y_{i}\right)\right) \tilde{u}\left(y_{1}\right) \prod_{j=1}^{N} d v\left(y_{j}\right)=\frac{\prod_{i=1}^{N} v\left(E_{i}\right)}{v\left(E_{1}\right)} \int_{E_{1}} \tilde{u}\left(y_{1}\right) d v\left(y_{1}\right) .
$$

PROOF. The equalities follow from simple calculations.

We will use the Landau big $\mathrm{O}$ notation in the sense that, given positive functions $g_{1}, g_{2}: \mathbb{R} \rightarrow \mathbb{R}$, the notation $g_{1}(\gamma) \leq(1+O(\gamma)) g_{2}(\gamma)$ means that there exist $C, \delta>0$ such that $g_{1}(\gamma) \leq(1+C \gamma) g_{2}(\gamma)$ when $0<\gamma<\delta$.

LEMMA 5.6. Let $\mathbf{S} \in \mathcal{S}_{\Omega}$. For all $i, j=1, \ldots, k, m \in S_{i}$ and $p \in S_{j}$, the following properties hold:

(i) If $m \neq p$, then

$$
\begin{aligned}
\int_{\pi^{-1}(\mathbf{S})} J_{S}\left(A_{m}\left(x_{m}\right), A_{p}\left(x_{p}\right)\right) d \mathbb{P}(\mathbf{x}) \\
\leq(1+O(\gamma)) \frac{\mathbb{P}\left(\pi^{-1}(\mathbf{S})\right)}{\mathcal{L}\left(F_{i}\right) \mathcal{L}\left(F_{j}\right)} \mathcal{L}\left(A_{m}\left(z_{i}\right)\right) \mathcal{L}\left(A_{p}\left(z_{j}\right)\right) J_{s}\left(F_{i}, F_{j}\right) .
\end{aligned}
$$

(ii) If $m=p$ (which implies that $i=j)$, then

$$
\int_{\pi^{-1}(\mathbf{S})} J_{s}\left(A_{m}\left(x_{m}\right), A_{p}\left(x_{p}\right)\right) d \mathbb{P}(\mathbf{x}) \leq(1+O(\gamma)) \mathbb{P}\left(\pi^{-1}(\mathbf{S})\right) I_{s}\left(A_{m}\left(z_{i}\right)\right) .
$$

PROOF. We begin by verifying (i). Recall that, by (5.11),

$$
\chi_{\pi^{-1}(\mathbf{S})}(\mathbf{x})=\prod_{i=1}^{k}\left(\prod_{m \in S_{i}} \chi_{F_{i, m}}\left(x_{m}\right)\right) \cdot\left(\prod_{n \in S_{i}^{*}} \chi_{\left(F_{i, n}\right)^{c}}\left(x_{n}\right)\right),
$$

where $S_{i}^{*}:=\left\{M_{i}, M_{i}+1, \ldots, M_{i+1}-1\right\} \backslash S_{i}$. Notice also that $\eta\left(F_{i, m}\right), \eta\left(F_{j, p}\right)>$ 0 by (5.7) and (5.4). Applying Lemma 5.5, we deduce

$$
\begin{aligned}
\int_{\pi^{-1}(\mathbf{S})} J_{S}\left(A_{m}\left(x_{m}\right), A_{p}\left(x_{p}\right)\right) d \mathbb{P}(\mathbf{x}) \\
=\frac{\mathbb{P}\left(\pi^{-1}(\mathbf{S})\right)}{\eta\left(F_{i, m}\right) \eta\left(F_{j, p}\right)} \iint_{F_{i, m} \times F_{j, p}} J_{S}\left(A_{m}(x), A_{p}(y)\right) d \eta(x) d \eta(y)
\end{aligned}
$$




$$
\begin{aligned}
\leq & \frac{(1+\gamma)^{2}}{(1-\gamma)^{2}} \frac{\mathbb{P}\left(\pi^{-1}(\mathbf{S})\right)}{\mathcal{L}\left(F_{i}\right) \mathcal{L}\left(F_{j}\right)} \\
& \times \iint_{F_{i, m} \times F_{j, p}} \iint_{A_{m}(x) \times A_{p}(y)}|u-v|^{-s} d \mathcal{L}(u) d \mathcal{L}(v) d \mathcal{L}(x) d \mathcal{L}(y)
\end{aligned}
$$

[by (5.6) and (5.7)]

$$
\leq \frac{(1+\gamma)^{5}}{(1-\gamma)^{2}} \frac{\mathbb{P}\left(\pi^{-1}(\mathbf{S})\right)}{\mathcal{L}\left(F_{i}\right) \mathcal{L}\left(F_{j}\right)} \mathcal{L}\left(A_{m}\left(z_{i}\right)\right) \mathcal{L}\left(A_{p}\left(z_{j}\right)\right) J_{s}\left(F_{i}, F_{j}\right) \quad \text { [by (5.8)]. }
$$

To prove (ii), we apply (5.6) and Lemma 5.5 to obtain

$$
\begin{aligned}
\int_{\pi^{-1}(\mathbf{S})} & J_{s}\left(A_{m}\left(x_{m}\right), A_{p}\left(x_{p}\right)\right) d \mathbb{P}(\mathbf{x}) \\
= & \frac{\mathbb{P}\left(\pi^{-1}(\mathbf{S})\right)}{\eta\left(F_{i, m}\right)} \int_{F_{i, m}} I_{s}\left(A_{m}(x)\right) d \eta(x) \\
\leq & (1+\gamma)^{2} \frac{\mathbb{P}\left(\pi^{-1}(\mathbf{S})\right)}{\mathcal{L}\left(F_{i, m}\right)} \int_{F_{i, m}} I_{s}\left(A_{m}\left(z_{i}\right)\right) d \mathcal{L}(x) \\
= & (1+\gamma)^{2} \mathbb{P}\left(\pi^{-1}(\mathbf{S})\right) I_{S}\left(A_{m}\left(z_{i}\right)\right) .
\end{aligned}
$$

Now we continue the proof of Proposition 5.4. Recalling (5.12) and (5.9), and applying Lemma 5.6, yields

$$
\begin{aligned}
\int_{\pi^{-1}(\mathbf{S})} & I_{S}\left(\mu^{\mathbf{x}}\right) d \mathbb{P}(\mathbf{x}) \\
\leq & \mathbb{P}\left(\pi^{-1}(\mathbf{S})\right)(1+O(\gamma))\left(\sum_{i=1}^{k} \sum_{j=1}^{k} c_{i} c_{j} J_{S}\left(F_{i}, F_{j}\right)+\sum_{i=1}^{k} c_{i}^{2} \mathcal{L}\left(F_{i}\right)^{2}\left(Q_{S_{i}}\right)^{-1}\right) \\
\leq & \mathbb{P}\left(\pi^{-1}(\mathbf{S})\right)(1+O(\gamma))\left(I_{S}(\mu)+\gamma\left(\sum_{i=1}^{k} c_{i} \mathcal{L}\left(F_{i}\right)\right)^{2}\right) \\
\leq & \mathbb{P}\left(\pi^{-1}(\mathbf{S})\right)(1+O(\gamma))\left(I_{S}(\mu)+\gamma\right) .
\end{aligned}
$$

Thus, by the choice of $\mu$, we have

$$
\begin{aligned}
\int_{\Omega} I_{S}\left(\mu^{\mathbf{x}}\right) d \mathbb{P}(\mathbf{x}) & =\sum_{\mathbf{S} \in \mathcal{S}_{\Omega}} \int_{\pi^{-1}(\mathbf{S})} I_{S}\left(\mu^{\mathbf{x}}\right) d \mathbb{P}(\mathbf{x}) \leq \mathbb{P}(\Omega)(1+O(\gamma))\left(I_{S}(\mu)+\gamma\right) \\
& \leq(1+O(\gamma))\left(\Gamma_{S}(F)+\frac{\varepsilon}{2}+\gamma\right) .
\end{aligned}
$$

The claim follows by choosing sufficiently small $\gamma$.

We complete this section by proving Theorem 1.1(b). 
Proof of TheOrem 1.1(B). We start by reducing the claim to the setting of Proposition 5.4. We may assume that $s_{0}(\mathbf{A})>0$. Consider $s<s_{0}(\mathbf{A})$. Since $G_{s}(E)=0$ for all $E \subset \mathbb{R}^{d}$ with $\mathcal{L}(E)=0$, we may assume that $\mathcal{L}\left(A_{n}\right)>0$ for all $n \in \mathbb{N}$ by removing sets with $\mathcal{L}\left(A_{n}\right)=0$ from the original sequence $\mathbf{A}=\left(A_{n}\right)_{n \in \mathbb{N}}$ if necessary. Since $I_{S}(E) \leq I_{S}(F)$ for $E \subset F$ and since $\mathcal{L}$ is inner regular, replacing $A_{n}$ by a suitable subset, we may assume that $A_{n}$ is compact for all $n \in \mathbb{N}$ and

$$
\sum_{n=1}^{\infty} g_{s}\left(A_{n}\right)=\infty
$$

We proceed by constructing a sequence $\left(K_{n}\right)_{n \in \mathbb{N}}$ of compact sets satisfying Definition 5.2(i)-(iv) such that $K_{n} \subset A_{n}$ for all $n \in \mathbb{N}$. Indeed, let $\left(Q_{i}\right)_{i=1}^{m_{1}}$ be the closed dyadic cubes with side length $2^{-1}$ intersecting $\Delta$. Notice that, for any Borel set $E \subset \Delta$, we have $E=\bigcup_{i=1}^{m_{1}} E \cap Q_{i}$ and, moreover, there exists $i \in\left\{1, \ldots, m_{1}\right\}$ with $\mathcal{L}\left(E \cap Q_{i}\right) \geq \frac{1}{m_{1}} \mathcal{L}(E)$. Thus

$$
\begin{aligned}
\sum_{i=1}^{m_{1}} g_{s}\left(E \cap Q_{i}\right) & =\sum_{i=1}^{m_{1}} \frac{\mathcal{L}\left(E \cap Q_{i}\right)^{2}}{I_{S}\left(E \cap Q_{i}\right)} \geq \sum_{i=1}^{m_{1}} \frac{\mathcal{L}\left(E \cap Q_{i}\right)^{2}}{I_{S}(E)} \\
& \geq \frac{\mathcal{L}(E)^{2}}{\left(m_{1}\right)^{2} I_{S}(E)}=\frac{1}{\left(m_{1}\right)^{2}} g_{s}(E) .
\end{aligned}
$$

It follows that

$$
\sum_{i=1}^{m_{1}} \sum_{j=1}^{\infty} g_{s}\left(A_{j} \cap Q_{i}\right)=\sum_{j=1}^{\infty} \sum_{i=1}^{m_{1}} g_{s}\left(A_{j} \cap Q_{i}\right) \geq \frac{1}{\left(m_{1}\right)^{2}} \sum_{j=1}^{\infty} g_{s}\left(A_{j}\right)=\infty .
$$

Therefore, there exists $k_{0} \in\left\{1, \ldots, m_{1}\right\}$ such that $\sum_{j=1}^{\infty} g_{s}\left(A_{j} \cap Q_{k_{0}}\right)=\infty$. Define $\widetilde{Q}_{1}:=Q_{k_{0}}$. We pick integers $n_{1}<n_{2}<\cdots<n_{N_{1}}$ so that

$$
\mathcal{L}\left(A_{n_{i}} \cap \widetilde{Q}_{1}\right)>0 \quad \text { for all } i=1, \ldots, N_{1} \quad \text { and } \quad \sum_{i=1}^{N_{1}} g_{s}\left(A_{n_{i}} \cap \widetilde{Q}_{1}\right) \geq 1 .
$$

Since $\sum_{j=N_{1}+1}^{\infty} g_{s}\left(A_{j} \cap \widetilde{Q}_{1}\right)=\infty$, a similar argument shows that there exist a dyadic cube $\widetilde{Q}_{2} \subset \widetilde{Q}_{1}$ with side length $2^{-2}$, and positive integers $n_{N_{1}+1}<\cdots<$ $n_{N_{2}}$ such that

$\mathcal{L}\left(A_{n_{i}} \cap \widetilde{Q}_{2}\right)>0 \quad$ for all $i=N_{1}+1, \ldots, N_{2} \quad$ and $\quad \sum_{i=N_{1}+1}^{N_{2}} g_{s}\left(A_{n_{i}} \cap \widetilde{Q}_{2}\right) \geq 1$.

Repeat this process inductively. As a result, we find a decreasing sequence $\left(\widetilde{Q}_{l}\right)_{l \in \mathbb{N}}$ of dyadic cubes, an increasing sequence $\left(N_{l}\right)_{l \in \mathbb{N}}$ of integers and an increasing sequence $\left(n_{l}\right)_{l \in \mathbb{N}}$ of indices such that, for every $k=0,1 \ldots$,

$$
\mathcal{L}\left(A_{n_{i}} \cap \widetilde{Q}_{k+1}\right)>0 \quad \text { for all } i=N_{k}+1, \ldots, N_{k+1} \quad \text { and }
$$




$$
\sum_{j=N_{k}+1}^{N_{k+1}} g_{s}\left(A_{n_{j}} \cap \widetilde{Q}_{k+1}\right) \geq 1 .
$$

Defining $K_{j}:=A_{n_{j}} \cap \widetilde{Q}_{k+1}$ for every $j=N_{k}+1, \ldots, N_{k+1}$, gives $\sum_{j=1}^{\infty} g_{s}\left(K_{j}\right)=$ $\infty$ and $\lim _{n \rightarrow \infty} \operatorname{diam} K_{n}=0$. Finally, setting $\left\{y_{0}\right\}:=\bigcap_{k=1}^{\infty} \widetilde{Q}_{k}$, leads to

$$
\lim _{n \rightarrow \infty} \operatorname{dist}\left(y_{0}, K_{n}\right)=0 \text {. }
$$

Hence the sequence $\left(K_{n}\right)_{n \in \mathbb{N}}$ satisfies items (i)-(iv) in Definition 5.2.

Since the measure $\sigma$ determining the probability $\mathbb{P}$ (recall Section 1 ) is not singular with respect to $\mathcal{L}$, there exists a compact set $E \subset U$ such that $\sigma(E)>0$, $\left.\sigma\right|_{E} \ll \mathcal{L}$ and (5.4) is satisfied with $h:=\frac{\left.d \sigma\right|_{E}}{d \mathcal{L}}$. Let $f: U \times V \rightarrow \mathbb{R}^{d}$ be as in the Introduction. For all $(x, y) \in U \times V$, let $T_{x}:=f(x, \cdot)^{-1}$ and $T^{y}:=f(\cdot, y)^{-1}$. Then, for all $x \in U$, the set $f\left(U, y_{0}\right) \cap f(x, V)$ is nonempty [it always contains the point $\left.f\left(x, y_{0}\right)\right]$ and open, $y_{0} \in V_{x}:=T_{x}\left(f\left(U, y_{0}\right) \cap f(x, V)\right)$ and $x \in U_{x}:=T^{y_{0}}\left(f\left(U, y_{0}\right) \cap f(x, V)\right)$. Thus the map $W_{x}: V_{x} \rightarrow U_{x}$ defined by $W_{x}(v):=T^{y_{0}}(f(x, v))$ is a diffeomorphism with $W_{x}\left(y_{0}\right)=x$ and

$$
\left\|D W_{x}\right\|,\left\|\left(D W_{x}\right)^{-1}\right\| \leq\left(C_{u}\right)^{2},
$$

where $C_{u}$ is as in inequality (1.13). Clearly, the derivative of the map $x \mapsto W_{x}(v)$ has the same bounds. Let $O$ be an open and bounded set such that $E \subset O \subset \bar{O} \subset$ $U$. Consider $0<r_{0}<\min \left\{\operatorname{dist}\left(y_{0}, V^{c}\right),\left(C_{u}\right)^{-2} \operatorname{dist}\left(\bar{O}, U^{c}\right)\right\}$. Then $B\left(y_{0}, r_{0}\right) \subset$ $V_{x}$ for all $x \in O$. Thus, $W: O \times B\left(y_{0}, r_{0}\right) \rightarrow \mathbb{R}^{d}$, defined by $W(x, y)=W_{x}(y)$, is a uniform bidiffeomorphism satisfying $W\left(x, y_{0}\right)=x$ for all $x \in O$ (recall Definition 5.2). Ignoring a finite number of sets $K_{n}$, if necessary, we may assume that $K_{n} \subset B\left(y_{0}, r_{0}\right)$ for all $n \in \mathbb{N}$. We conclude that all the conditions in Definition 5.2 are fulfilled.

As a result of the fact that $T^{y_{0}}$ is a diffeomorphism, we conclude that

$$
\operatorname{dim}_{\mathrm{H}}\left(\limsup _{n \rightarrow \infty} f\left(x_{n}, K_{n}\right)\right) \geq \operatorname{dim}_{\mathrm{H}}\left(\limsup _{n \rightarrow \infty} W\left(x_{n}, K_{n}\right)\right)
$$

for all $\mathbf{x} \in U^{\mathbb{N}}$. Here, we have an inequality instead of an equality since for $x_{n} \in$ $U \backslash O$ it may happen that $K_{n} \not \subset V_{x_{n}}$. Finally, the claim follows by combining Proposition 5.4, Lemma 4.7 and Proposition 4.5.

6. Packing dimension of random covering sets. In this section, we prove Theorem 1.1(d). For the purpose of studying packing dimensions of random covering sets, we set

$$
N_{\ell}^{*}(E):=\#\left\{Q \in \mathcal{Q}_{\ell}: \mathcal{L}(Q \cap E)>0\right\}
$$

for all $E \subset \mathbb{R}^{d}$ and $\ell \in \mathbb{N}$. Here, the symbol \# stands for the cardinality and $\mathcal{Q}_{\ell}$ is as in (3.2). We begin with a result concerning a lower bound for packing dimensions of intersections of decreasing sequences of compact sets. 
LEMMA 6.1. Let $\left(E_{n}\right)_{n \in \mathbb{N}}$ be a decreasing sequence of compact subsets of $\mathbb{R}^{d}$ with positive Lebesgue measure. Let $s>0$. Assume that there exists a sequence $\left(\ell_{n}\right)_{n \in \mathbb{N}}$ of natural numbers such that

$$
N_{\ell_{n+1}}^{*}\left(Q \cap E_{n+1}\right) \geq 2^{\ell_{n+1} s}
$$

for all $n \in \mathbb{N}$ and for all $Q \in \mathcal{Q}_{\ell_{n}}$ with $\mathcal{L}\left(Q \cap E_{n}\right)>0$. Then $\operatorname{dim}_{P}\left(\cap_{n=1}^{\infty} E_{n}\right) \geq s$.

Proof. For $n \in \mathbb{N}$, set

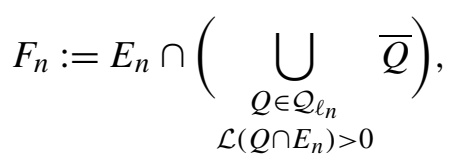

and let $F_{\infty}:=\bigcap_{n=1}^{\infty} F_{n}$. Clearly, $F_{n} \subset E_{n}$ is compact and $\mathcal{L}\left(F_{n}\right)=\mathcal{L}\left(E_{n}\right)$. Hence $N_{\ell_{n}}^{*}\left(Q \cap F_{n}\right)=N_{\ell_{n}}^{*}\left(Q \cap E_{n}\right)$ for all $n \in \mathbb{N}$ and $Q \in \bigcup_{k=1}^{\infty} \mathcal{Q}_{k}$. In particular, we have

$$
N_{\ell_{n+1}}^{*}\left(Q \cap F_{n+1}\right) \geq 2^{\ell_{n+1} s}
$$

for all $n \in \mathbb{N}$ and $Q \in \mathcal{Q}_{\ell_{n}}$ with $\mathcal{L}\left(Q \cap F_{n}\right)>0$. Denoting by $\overline{\operatorname{dim}}_{\mathrm{B}}$ the upper box counting dimension, we will show that

$$
\overline{\operatorname{dim}}_{\mathrm{B}}\left(V \cap F_{\infty}\right) \geq s
$$

for all open sets $V$ with $V \cap F_{\infty} \neq \varnothing$. By the Baire category theorem, (6.3) implies that $\operatorname{dim}_{P}\left(F_{\infty}\right) \geq s$ (see, e.g., [21], Proposition 3.6 and Corollary 3.9) and, therefore, $\operatorname{dim}_{\mathrm{P}}\left(\bigcap_{n=1}^{\infty} E_{n}\right) \geq s$, as desired.

To prove (6.3), let $V$ be an open set so that $V \cap F_{\infty} \neq \varnothing$. Then there exist $n \in \mathbb{N}$ and $Q \in \mathcal{Q}_{\ell_{n}}$ such that $3 Q \subset V$ and $Q \cap F_{n} \neq \varnothing$, where $3 Q$ stands for the union of all elements $Q^{\prime} \in \mathcal{Q}_{\ell_{n}}$ with $\overline{Q^{\prime}} \cap \bar{Q} \neq \varnothing$. By the definition of $F_{n}$, there is $Q^{*} \in \mathcal{Q}_{\ell_{n}}$ such that $\overline{Q^{*}} \cap \bar{Q} \neq \varnothing$ and $\mathcal{L}\left(Q^{*} \cap F_{n}\right)>0$. Since $Q^{*} \subset 3 Q \subset V$, replacing $Q$ by $Q^{*}$, if necessary, we may assume that $\mathcal{L}\left(Q \cap F_{n}\right)>0$. Using (6.2) recursively, leads to

$$
N_{\ell_{m}}^{*}\left(Q \cap F_{m}\right) \geq 2^{\ell_{m} s}
$$

for all $m>n$. Furthermore, we claim that, for every $m>n$,

$$
\#\left\{Q^{\prime} \in \mathcal{Q}_{\ell_{m}}: \overline{Q^{\prime}} \cap Q \cap F_{\infty}\right\} \geq N_{\ell_{m}}^{*}\left(Q \cap F_{m}\right) \geq 2^{\ell_{m} s},
$$

from which we conclude that $\overline{\operatorname{dim}}_{\mathrm{B}}\left(Q \cap F_{\infty}\right) \geq s$ and, therefore, $\overline{\operatorname{dim}}_{\mathrm{B}}\left(V \cap F_{\infty}\right) \geq$ $s$. To prove (6.5), it follows from (6.4) that it is enough to show that $\overline{Q^{\prime}} \cap F_{\infty} \neq$ $\varnothing$ for all $Q^{\prime} \in \mathcal{Q}_{\ell_{m}}$ with $\mathcal{L}\left(Q^{\prime} \cap F_{m}\right)>0$. For this purpose, consider $Q^{\prime} \in \mathcal{Q}_{\ell_{m}}$ with $\mathcal{L}\left(Q^{\prime} \cap F_{m}\right)>0$. By (6.2), there exists $Q_{1}^{\prime} \in \mathcal{Q}_{\ell_{m+1}}$ such that $Q_{1}^{\prime} \subset Q^{\prime}$ and $\mathcal{L}\left(Q_{1}^{\prime} \cap F_{m+1}\right)>0$. Using this fact recursively, we see that, for every $p \in \mathbb{N}$, there exists $Q_{p}^{\prime} \in \mathcal{Q}_{\ell_{m+p}}$ such that $Q_{p}^{\prime} \subset Q_{p-1}^{\prime}$ and $\mathcal{L}\left(Q_{p}^{\prime} \cap F_{m+p}\right)>0$. Hence, we have 
$\mathcal{L}\left(Q^{\prime} \cap F_{m+p}\right)>0$ for all $p \in \mathbb{N}$, which implies that $\overline{Q^{\prime}} \cap F_{\infty} \neq \varnothing$. This completes the proof of the lemma.

Before applying the above result to estimate packing dimensions of random covering sets, we prove several lemmas.

LEMmA 6.2. For all $A \in \mathcal{B}\left(\mathbb{R}^{d}\right)$ and $\ell \in \mathbb{Z}$, we have $N_{\ell}^{*}(A) \geq 2^{\ell d} \mathcal{L}(A)$.

PROOF. The claim follows directly from a simple volume argument.

Let $U \subset \mathbb{R}^{d}$ be open, and let $\left(A_{n}(x)\right)_{n \in \mathbb{N}}$ be a sequence of compact-set-valued functions defined on $U$ satisfying the conditions $(\mathrm{C}-1)$ and $(\mathrm{C}-2)$ from Section 4. Let $\eta \in \mathcal{P}(U)$ and set $\mathbb{P}:=\prod_{i=1}^{\infty} \eta$.

LEMMA 6.3. Let $E \in \mathcal{B}\left(\mathbb{R}^{d}\right)$ with $0<\mathcal{L}(E)<\infty$, and let $\ell \in \mathbb{Z}$. Then the mapping

$$
\left(x_{i}\right)_{i=1}^{n} \mapsto N_{\ell}^{*}\left(E \cap \bigcup_{i=1}^{n} A_{i}\left(x_{i}\right)\right)
$$

is lower semicontinuous on $U^{n}$ for all $n \in \mathbb{N}$. Moreover, the mapping

$$
\mathbf{x} \mapsto N_{\ell}^{*}\left(E \cap \bigcup_{i=1}^{\infty} A_{i}\left(x_{i}\right)\right)
$$

is Borel measurable on $U^{\mathbb{N}}$.

PROOF. It suffices to prove the first part of the lemma; the second part follows directly from the first one and the following easily-checked identity:

$$
N_{\ell}^{*}\left(E \cap \bigcup_{i=1}^{\infty} A_{i}\left(x_{i}\right)\right)=\lim _{n \rightarrow \infty} N_{\ell}^{*}\left(E \cap \bigcup_{i=1}^{n} A_{i}\left(x_{i}\right)\right) .
$$

Let $\left(x_{i}\right)_{i=1}^{n} \in U^{n}$ and write $k:=N_{\ell}^{*}\left(E \cap \bigcup_{i=1}^{n} A_{i}\left(x_{i}\right)\right)$ for short. Then there are $k$ different elements in $\mathcal{Q}_{\ell}$, say $Q_{1}, \ldots, Q_{k}$, such that $\mathcal{L}\left(Q_{j} \cap \bigcup_{i=1}^{n} A_{i}\left(x_{i}\right)\right)>0$ for all $j=1, \ldots, k$. It follows from $(\mathrm{C}-2)$ that $\mathcal{L}\left(\bigcup_{i=1}^{n} A_{i}\left(x_{i}\right) \backslash \bigcup_{j=1}^{n} A_{j}\left(y_{j}\right)\right)$ is close to 0 when $\left(y_{i}\right)_{i=1}^{n} \in U^{n}$ is close to $\left(x_{i}\right)_{i=1}^{n}$ and, therefore, when $\left(y_{i}\right)_{i=1}^{n}$ is in a small neighbourhood of $\left(x_{i}\right)_{i=1}^{n}$, we have $\mathcal{L}\left(Q_{j} \cap \bigcup_{i=1}^{n} A_{i}\left(y_{i}\right)\right)>0$ for all $j=1, \ldots, k$. Hence $N_{\ell}^{*}\left(E \cap \bigcup_{i=1}^{n} A_{i}\left(y_{i}\right)\right) \geq k$, concluding the proof of lower semicontinuity.

The following result may be regarded as an analogy of Proposition 4.5. 
Proposition 6.4. Let $E \subset U$ be compact with $\eta(E)>0$. Suppose that $\left.\eta\right|_{E} \ll \mathcal{L}$. Moreover, assume that, for every $\ell, n \in \mathbb{N}$ and for every compact set $F \subset E$ with $\eta(F)>0$,

$$
N_{\ell}^{*}\left(F \cap \bigcup_{i=n}^{\infty} A_{i}\left(x_{i}\right)\right)=N_{\ell}^{*}(F)
$$

for $\mathbb{P}$-almost all $\mathbf{x} \in U^{\mathbb{N}}$. Then

$$
\operatorname{dim}_{\mathrm{P}}\left(\limsup _{n \rightarrow \infty} A_{n}\left(x_{n}\right)\right)=d
$$

for $\mathbb{P}$-almost all $\mathbf{x} \in U^{\mathbb{N}}$.

PROOF. Replacing $E$ by a compact subset, if necessary, we may assume that $0<\frac{\left.d \eta\right|_{E}}{d \mathcal{L}}(x)<\infty$ for all $x \in E$. Thus, for all $F \subset E$, we have

$$
\mathcal{L}(F)>0 \quad \text { if and only if } \eta(F)>0 .
$$

Let $\varepsilon, \delta>0$. It suffices to verify that

$$
\mathbb{P}\left(\left\{\mathbf{x} \in U^{\mathbb{N}}: \operatorname{dim}_{\mathrm{P}}\left(\limsup _{n \rightarrow \infty} A_{n}\left(x_{n}\right)\right) \geq d-\delta\right\}\right) \geq 1-\varepsilon .
$$

For this purpose, we are going to construct a Borel set $\Omega \subset U^{\mathbb{N}}$ with $\mathbb{P}(\Omega)>1-\varepsilon$, and two sequences $\left(\ell_{k}\right)_{k \in \mathbb{N}}$ and $\left(m_{k}\right)_{k \in \mathbb{N}}$ of natural numbers such that, for all $\mathbf{x} \in$ $\Omega, k \in \mathbb{N}$ and $Q \in \mathcal{Q}_{\ell_{k}}$, we have

$$
N_{\ell_{k+1}}^{*}\left(Q \cap E \cap \bigcap_{j=1}^{k+1} \bigcup_{i=m_{j}+1}^{m_{j+1}} A_{i}\left(x_{i}\right)\right) \geq 2^{\ell_{k+1}(d-\delta)}
$$

provided that $\mathcal{L}\left(Q \cap E \cap \bigcap_{j=1}^{k} \bigcup_{i=m_{j}+1}^{m_{j+1}} A_{i}\left(x_{i}\right)\right)>0$. By Lemma 6.1, this implies that

$$
\operatorname{dim}_{\mathrm{P}}\left(\limsup _{n \rightarrow \infty} A_{n}\left(x_{n}\right)\right) \geq d-\delta
$$

for all $\mathbf{x} \in \Omega$, from which (6.9) follows.

Now we present our construction. Set $\ell_{1}:=1$ and $m_{1}:=1$. Notice that

$$
\gamma_{1}:=\min \left\{\mathcal{L}(Q \cap E): Q \in \mathcal{Q}_{\ell_{1}} \text { and } \mathcal{L}(Q \cap E)>0\right\}>0 .
$$

Choosing a large integer $\ell_{2}>\ell_{1}$ so that $2^{-\ell_{2} \delta}<\gamma_{1}$, it follows from Lemma 6.2 that

$$
N_{\ell_{2}}^{*}(Q \cap E) \geq 2^{\ell_{2} d} \gamma_{1}>2^{\ell_{2}(d-\delta)}
$$

for all $Q \in \mathcal{Q}_{\ell_{1}}$ with $\mathcal{L}(Q \cap E)>0$. Hence, by (6.7), for $\mathbb{P}$-almost all $\mathbf{x} \in U^{\mathbb{N}}$ and for all $Q \in \mathcal{Q}_{\ell_{1}}$ with $\mathcal{L}(Q \cap E)>0$, we have

$$
N_{\ell_{2}}^{*}\left(Q \cap E \cap \bigcup_{i=m_{1}+1}^{\infty} A_{i}\left(x_{i}\right)\right)=N_{\ell_{2}}^{*}(Q \cap E)>2^{\ell_{2}(d-\delta)},
$$


where we used (6.8) and the fact that $N_{\ell}^{*}(\bar{Q} \cap A)=N_{\ell}^{*}(Q \cap A)$ for all $\ell \in \mathbb{N}$, $Q \in \mathcal{Q}_{\ell}$ and $A \subset \mathbb{R}^{d}$. By (6.6) and Lemma 6.3, we find a large integer $m_{2}>m_{1}$ and a Borel set $\Lambda_{2} \subset U^{m_{2}}$ with $\eta^{m_{2}}\left(\Lambda_{2}\right)>1-\frac{\varepsilon}{2}$ such that, for all $\left(x_{1}, \ldots, x_{m_{2}}\right) \in \Lambda_{2}$,

$$
N_{\ell_{2}}^{*}\left(Q \cap E \cap \bigcup_{i=m_{1}+1}^{m_{2}} A_{i}\left(x_{i}\right)\right)>2^{\ell_{2}(d-\delta)}
$$

for all $Q \in \mathcal{Q}_{\ell_{1}}$ with $\mathcal{L}(Q \cap E)>0$. Define a mapping $\tau_{2}: \Lambda_{2} \rightarrow(0, \infty)$ by

$$
\begin{aligned}
\tau_{2}\left(x_{1}, \ldots, x_{m_{2}}\right):= & \min \left\{\mathcal{L}\left(Q \cap E \cap\left(\bigcup_{i=m_{1}+1}^{m_{2}} A_{i}\left(x_{i}\right)\right)\right): Q \in \mathcal{Q}_{\ell_{2}}\right. \\
& \text { with } \left.\mathcal{L}\left(Q \cap E \cap\left(\bigcup_{i=m_{1}+1}^{m_{2}} A_{i}\left(x_{i}\right)\right)\right)>0\right\} .
\end{aligned}
$$

By (C-2), the function $\tau_{2}$ is continuous, and hence, Borel measurable on $\Lambda_{2}$. Since $\tau_{2}\left(x_{1}, \ldots, x_{m_{2}}\right)>0$ for all $\left(x_{1}, \ldots, x_{m_{2}}\right) \in \Lambda_{2}$, there exist $\gamma_{2}>0$ and a Borel set $\Lambda_{2}^{\prime} \subset \Lambda_{2}$ such that

$$
\eta^{m_{2}}\left(\Lambda_{2}^{\prime}\right)>\eta^{m_{2}}\left(\Lambda_{2}\right)-\frac{\varepsilon}{6}>1-\frac{2 \varepsilon}{3}
$$

and

$$
\tau_{2}\left(x_{1}, \ldots, x_{m_{2}}\right) \geq \gamma_{2}
$$

for all $\left(x_{1}, \ldots, x_{m_{2}}\right) \in \Lambda_{2}^{\prime}$. Choose $\ell_{3}>\ell_{2}$ so that $2^{-\ell_{3} \delta}<\gamma_{2}$. Lemma 6.2 implies that, for all $\left(x_{1}, \ldots, x_{m_{2}}\right) \in \Lambda_{2}^{\prime}$ and $Q \in \mathcal{Q}_{\ell_{3}}$,

$$
N_{\ell_{3}}^{*}\left(Q \cap E \cap\left(\bigcup_{i=m_{1}+1}^{m_{2}} A_{i}\left(x_{i}\right)\right)\right) \geq 2^{\ell_{3} d} \gamma_{2}>2^{\ell_{3}(d-\delta)}
$$

provided that $\mathcal{L}\left(Q \cap E \cap\left(\bigcup_{i=m_{1}+1}^{m_{2}} A_{i}\left(x_{i}\right)\right)\right)>0$. Again, by (6.7), we find $m_{3}>m_{2}$ and a Borel set $\Lambda_{3} \subset \Lambda_{2}^{\prime} \times \prod_{i=m_{2}+1}^{m_{3}} U \subset \Lambda_{2} \times \prod_{i=m_{2}+1}^{m_{3}} U \subset U^{m_{3}}$ such that

$$
\eta^{m_{3}}\left(\Lambda_{3}\right)>\eta^{m_{2}}\left(\Lambda_{2}^{\prime}\right)-\frac{\varepsilon}{12}>1-\frac{3 \varepsilon}{4}
$$

and, moreover, for all $\left(x_{1}, \ldots, x_{m_{3}}\right) \in \Lambda_{m_{3}}$ and $Q \in \mathcal{Q}_{\ell_{3}}$,

$$
N_{\ell_{3}}^{*}\left(Q \cap E \cap \bigcap_{j=1}^{2}\left(\bigcup_{i=m_{j}+1}^{m_{j+1}} A_{i}\left(x_{i}\right)\right)\right)>2^{\ell_{3}(d-\delta)}
$$

provided that $\mathcal{L}\left(Q \cap E \cap\left(\bigcup_{i=m_{1}}^{m_{2}} A_{i}\left(x_{i}\right)\right)\right)>0$.

Continuing the above process, we construct recursively two increasing sequences $\left(\ell_{k}\right)_{k \in \mathbb{N}}$ and $\left(m_{k}\right)_{k \in \mathbb{N}}$ of integers and a sequence $\left(\Lambda_{k}\right)_{k \in \mathbb{N}}$ of Borel sets 
such that $\Lambda_{k} \subset U^{m_{k}}, \Lambda_{k+1} \subset \Lambda_{k} \times \prod_{i=m_{k}+1}^{m_{k+1}} U, \eta^{m_{k}}\left(\Lambda_{k}\right)>1-\frac{(2 k-1) \varepsilon}{2 k}$ and inequality (6.10) holds for all $\left(x_{1}, \ldots, x_{m_{k+1}}\right) \in \Lambda_{k+1}$. Setting $\Omega:=\bigcap_{k=1}^{\infty}\left(\Lambda_{k} \times\right.$ $\prod_{i=m_{k}+1}^{\infty} U$ ), gives $\mathbb{P}(\Omega)=\lim _{k \rightarrow \infty} \eta^{m_{k}}\left(\Lambda_{k}\right) \geq 1-\varepsilon$ and, moreover, (6.10) holds for all $\mathbf{x} \in \Omega$. This completes the proof.

Now we are ready to prove our main result on the packing dimension of random covering sets.

THEOREM 6.5. Let $E \subset \mathbb{R}^{d}$ be compact with $\eta(E)>0$. Suppose that $\left.\eta\right|_{E}$ is equivalent with $\left.\mathcal{L}\right|_{E}$. Let $\left(A_{n}(x)\right)_{n \in \mathbb{N}}$ be a sequence of compact-set-valued functions defined on $U$ satisfying the conditions $(\mathrm{C}-1)$ and $(\mathrm{C}-2)$ from Section 4 . In addition, suppose that, for all compact sets $F \subset E$ with $\mathcal{L}(F)>0$,

$$
\sum_{n=1}^{\infty} \eta\left(\left\{x \in F: \mathcal{L}\left(F \cap A_{n}(x)\right)>0\right\}\right)=\infty .
$$

Then, for $\mathbb{P}$-almost all $\mathbf{x} \in U^{\mathbb{N}}$,

$$
\operatorname{dim}_{\mathrm{P}}\left(\limsup _{n \rightarrow \infty} A_{n}\left(x_{n}\right)\right)=d
$$

Proof. Let $\ell \in \mathbb{N}$, and let $F \subset E$ be compact with $\mathcal{L}(F)>0$. By Proposition 6.4, it is sufficient to prove that, for all $n \in \mathbb{N}$,

$$
N_{\ell}^{*}\left(F \cap \bigcup_{i=n}^{\infty} A_{i}\left(x_{i}\right)\right)=N_{\ell}^{*}(F)
$$

for $\mathbb{P}$-almost all $\mathbf{x} \in U^{\mathbb{N}}$. Note that (6.13) is equivalent to the statement that, for all $Q \in \mathcal{Q}_{\ell}$ with $\mathcal{L}(Q \cap F)>0$,

$$
\mathcal{L}\left(Q \cap F \cap \bigcup_{i=n}^{\infty} A_{i}\left(x_{i}\right)\right)>0 \quad \text { for } \mathbb{P} \text {-almost all } \mathbf{x} \in U^{\mathbb{N}} .
$$

Fix $Q \in \mathcal{Q}_{\ell}$ with $\mathcal{L}(Q \cap F)>0$. For all $k \in \mathbb{N}$, we consider the independent events

$$
E_{k}:=\left\{x_{k} \in Q \cap F: \mathcal{L}\left(Q \cap F \cap A_{k}\left(x_{k}\right)\right)>0\right\} .
$$

Replacing $F$ by $\bar{Q} \cap F$ in (6.12), we have $\sum_{k=1}^{\infty} \eta\left(E_{k}\right)=\infty$. Applying the second Borel-Cantelli lemma, yields (6.14).

We complete this section by proving Theorem 1.1(d).

Proof of TheOREM 1.1(D). Recall from the Introduction that $A_{n}\left(x_{n}\right)=$ $f\left(x_{n}, A_{n}\right)$. Since $\mathcal{L}$ is inner regular, we may assume that the sets $A_{n}$ are compact with $\mathcal{L}\left(A_{n}\right)>0$ and properties $(\mathrm{C}-1)$ and $(\mathrm{C}-2)$ are satisfied. Let $F \subset U$ be a compact set with $\mathcal{L}(F)>0$ such that $\left.\sigma\right|_{E}$ is equivalent with $\left.\mathcal{L}\right|_{F}$. As in the proof of Theorem 1.1(b), we may replace $f\left(x_{n}, A_{n}\right)$ by $W\left(x_{n}, A_{n}\right)$. Then (6.12) follows from Lemma 2.3. Hence Theorem 6.5 implies the claim. 
7. Proof of Corollary 1.2 and examples. The aim of this section is to verify Corollary 1.2 and to discuss the sharpness of our results. We begin by proving Corollary 1.2 as a consequence of Theorem 1.1.

Proof of Corollary 1.2. Observe that, for all sequences $\left(E_{n}\right)_{n \in \mathbb{N}}$ and $\left(F_{n}\right)_{n \in \mathbb{N}}$ of subsets of $\mathbf{N}$, we have $\limsup _{n \rightarrow \infty}\left(E_{n} \cup F_{n}\right)=\left(\limsup _{n \rightarrow \infty} E_{n}\right) \cup$ (limsup $\operatorname{su}_{n \rightarrow \infty} F_{n}$ ). Therefore, covering $\mathbf{M}$ and $\mathbf{N}$ by a finite number of coordinate charts, we may assume that $f: \mathbf{K} \times V \rightarrow \mathbb{R}^{d}$ satisfies the assumptions in Theorem 1.1 with the exception that $f(\cdot, y)$ is not necessarily injective. Recall that as long as (1.13) is valid, the dependence of $f$ on the first coordinate plays no role when proving that $t_{0}(\mathbf{A})$ is an upper bound for the dimension and $s_{0}(\mathbf{A})=t_{0}(\mathbf{A})$ (see Section 3). In Sections 4-6, where the lower bounds are proven, we restrict our considerations into a bounded set where $\sigma$ is absolutely continuous with respect to the Lebesgue measure. We deduce that, covering $\mathbf{K}$ by a finite number of coordinate charts, we may assume that $f: U \times V \rightarrow \mathbb{R}^{d}$ is as in Theorem 1.1 and, therefore, Corollary 1.2 follows from Theorem 1.1.

We continue by constructing examples that demonstrate the sharpness of our results. We begin with showing that the lower bound proven by Persson [see (1.3)] is not always sharp.

EXAMPLE 7.1. Let $Q_{1}, Q_{2} \subset \mathbb{R}^{d}$ be disjoint open cubes with side lengths $r_{1}$ and $r_{2}$, respectively. Let $0<\rho<1$. Divide $Q_{2}$ into $2^{\text {nd }}$ subcubes $Q_{2}^{j}$ and set $F_{Q_{2}}:=\bigcup_{j=1}^{2 n d} \rho Q_{2}^{j}$, where $\rho Q$ is the concentric cube with $Q$ having side length $\rho$ times that of $Q$. Define $A:=Q_{1} \cup F_{Q_{2}}$. Using the change of variables $x^{\prime}=r_{i} x$ for $i=1,2$, one easily sees that $I_{t}\left(Q_{1}\right)=C_{1} r_{1}^{-t} \mathcal{L}\left(Q_{1}\right)^{2}$ and $I_{t}\left(F_{Q_{2}}\right) \leq C_{2} r_{2}^{-t} \mathcal{L}\left(F_{Q_{2}}\right)^{2}$, where $C_{1}$ and $C_{2}$ are constants depending only on $d$ and $t$. Choosing sufficiently small $\rho>0$, guarantees that $\mathcal{L}(A)<2 \mathcal{L}\left(Q_{1}\right)$ which, in turn, implies that

$$
\frac{g_{t}(A)}{g_{t}\left(F_{Q_{2}}\right)} \leq \frac{4 \mathcal{L}\left(Q_{1}\right)^{2}}{I_{t}\left(Q_{1}\right)} \frac{I_{t}\left(F_{Q_{2}}\right)}{\mathcal{L}\left(F_{Q_{2}}\right)^{2}} \leq C\left(\frac{r_{1}}{r_{2}}\right)^{t},
$$

where $C$ is a constant. Hence, $G_{t}(A) \geq g_{t}\left(F_{Q_{2}}\right) \geq C^{-1}\left(\frac{r_{2}}{r_{1}}\right)^{t} g_{t}(A)$. Since the ratio $\frac{r_{2}}{r_{1}}$ can be chosen arbitrarily large, we conclude that even for open sets and for $\stackrel{r_{1}}{\sigma}:=\mathcal{L}$, the lower bound given for $\operatorname{dim}_{\mathrm{H}} \mathbf{E}(\mathbf{x}, \mathbf{A})$ in [47] by means of $g_{t}$ may be strictly smaller than the quantity $s_{0}(\mathbf{A})$ in Theorem 1.1 [see (1.8)].

Next, we give an example which shows that if we replace the assumption that every $A_{n}$ has positive Lebesgue density by a weaker assumption that $\mathcal{L}\left(A_{n} \cap\right.$ $B(x, r))>0$ for all $n \in \mathbb{N}, x \in A_{n}$ and $r>0$, Theorem 1.1(c) is not valid and $\operatorname{dim}_{\mathrm{H}} \mathbf{E}(\mathbf{x}, \mathbf{A})$ can be almost surely strictly smaller than $t_{0}(\mathbf{A})$. 
EXAMPLE 7.2. Let $\sigma:=\mathcal{L}$ on $\mathbb{T}^{2}$ and set $\mathbb{P}:=\prod_{i=1}^{\infty} \sigma$. Define $f(x, y): \mathbb{T}^{2} \times$ $\mathbb{T}^{2} \rightarrow \mathbb{T}^{2}$ by $f(x, y)=x+y$ for all $(x, y) \in \mathbb{T}^{2} \times \mathbb{T}^{2}$. For every $n \in \mathbb{N}$, let $E_{n}:=[0,1] \times\{0\} \subset \mathbb{T}^{2}$ and $F_{n}:=\bigcup_{i=1}^{\infty} B\left(y_{i}, 2^{-n-i}\right) \subset \mathbb{T}^{2}$ where the centres $y_{i}$ are dense in $E_{n}$. Set $A_{n}:=E_{n} \cup F_{n}$ and write $\mathbf{A}:=\left(A_{n}\right)_{n \in \mathbb{N}}, \mathbf{E}:=\left(E_{n}\right)_{n \in \mathbb{N}}$ and $\mathbf{F}:=\left(F_{n}\right)_{n \in \mathbb{N}}$. We deduce that $\mathcal{L}\left(A_{n} \cap B(y, r)\right)>0$ for all $r>0$ and $y \in A_{n}$ but

$$
\liminf _{r \rightarrow 0} \frac{\mathcal{L}\left(A_{n} \cap B(y, r)\right)}{\mathcal{L}(B(y, r))}=0
$$

for $\mathcal{H}^{1}$-almost all $y \in E_{n} \backslash F_{n}$, which follows by applying the Lebesgue density theorem for $\left.\mathcal{H}^{1}\right|_{E_{n}}$ and noting that $\mathcal{L}\left(A_{n} \cap B(y, r)\right) \leq 2 r \mathcal{H}^{1}\left(B(y, r) \cap E_{n} \cap F_{n}\right)$. Recall that

$$
\limsup _{n \rightarrow \infty}\left(x_{n}+A_{n}\right)=\limsup _{n \rightarrow \infty}\left(x_{n}+E_{n}\right) \cup \limsup _{n \rightarrow \infty}\left(x_{n}+F_{n}\right) .
$$

Now $\sum_{n=1}^{\infty} \mathcal{H}_{\infty}^{1}\left(E_{n}\right)=\infty$ and $\sum_{n=1}^{\infty} \mathcal{H}_{\infty}^{t}\left(F_{n}\right)<\infty$ for all $t>0$. Thus $t_{0}(\mathbf{A})=1$ and $t_{0}(\mathbf{F})=0$. By Corollary 1.2, we have $\operatorname{dim}_{\mathrm{H}}\left(\lim \sup _{n \rightarrow \infty}\left(x_{n}+F_{n}\right)\right)=0$ for all $\mathbf{x} \in\left(\mathbb{T}^{2}\right)^{\mathbb{N}}$. Furthermore, lim $\sup _{n \rightarrow \infty}\left(x_{n}+E_{n}\right)=\varnothing \mathbb{P}$-almost surely, since

$$
\mathbb{P}\left(\left(x_{n}+E_{n}\right) \cap\left(x_{m}+E_{m}\right) \neq \varnothing \text { for some } n, m \in \mathbb{N} \text { with } n \neq m\right)=0 .
$$

We conclude that $\operatorname{dim}_{\mathrm{H}}\left(\lim \sup _{n \rightarrow \infty}\left(x_{n}+A_{n}\right)\right)=0<1=t_{0}(\mathbf{A}) \mathbb{P}$-almost surely. Observe that $s_{0}(\mathbf{A})=s_{0}(\mathbf{F})=0$.

Next, we construct an example illustrating that if the generating sets $A_{n}$ do not have positive Lebesgue density it is possible that $\operatorname{dim}_{H} \mathbf{E}(\mathbf{x}, \mathbf{A})>s_{0}(\mathbf{A})$ almost surely. For this purpose, we recall the following notation from [20].

DeFinition 7.3. For all $0<s \leq d$, let

$$
\begin{aligned}
\mathcal{G}^{s}\left(\mathbb{R}^{d}\right):= & \left\{F \subset \mathbb{R}^{d}: F \text { is a } G_{\delta} \text {-set such that } \operatorname{dim}_{\mathrm{H}}\left(\bigcap_{i=1}^{\infty} f_{i}(F)\right) \geq s\right. \text { for all } \\
& \text { similarities } \left.f_{i}: \mathbb{R}^{d} \rightarrow \mathbb{R}^{d}, i \in \mathbb{N}\right\} .
\end{aligned}
$$

We say that the sets in the class $\mathcal{G}^{s}\left(\mathbb{R}^{d}\right)$ have large intersection property.

In [20], Theorem A, Falconer showed that $\mathcal{G}^{s}\left(\mathbb{R}^{d}\right)$ is the maximal class of $G_{\delta^{-}}$ sets of Hausdorff dimension at least $s$ which is closed under countable intersections and similarities. Moreover, in [20], Theorem B, he gave several equivalent ways to define the class $\mathcal{G}^{s}\left(\mathbb{R}^{d}\right)$, one of them being

$$
F \in \mathcal{G}^{s}\left(\mathbb{R}^{d}\right) \quad \Longleftrightarrow \mathcal{M}_{\infty}^{s}(F \cap Q)=\mathcal{M}_{\infty}^{s}(Q)
$$

for all dyadic cubes $Q$, 
where $\mathcal{M}_{\infty}^{s}$ is the $s$-dimensional net content defined as in (1.6) with covering sets being dyadic cubes. Definition (7.1) was extended by Bugeaud [9] and Durand [13] for general gauge functions and open subsets of $\mathbb{R}^{d}$.

EXAmple 7.4. Let $\sigma:=\mathcal{L}$ on $\mathbb{T}^{d}, \mathbb{P}:=\prod_{i=1}^{\infty} \sigma$ and define $f: \mathbb{T}^{d} \times \mathbb{T}^{d} \rightarrow \mathbb{T}^{d}$ by $f(x, y)=x+y$ for all $(x, y) \in \mathbb{T}^{d} \times \mathbb{T}^{d}$. Consider $0<s<t<d$ and choose a sequence $\left(\widetilde{B}_{i}\right)_{i \in \mathbb{N}}$ of open balls such that $\operatorname{dim}_{\mathrm{H}} \mathbf{E}\left(\mathbf{x},\left(\widetilde{B}_{i}\right)_{i \in \mathbb{N}}\right)=t$ for $\mathbb{P}$-almost all $\mathbf{x} \in\left(\mathbb{T}^{d}\right)^{\mathbb{N}}$. Fix such a typical covering set and denote it by $F$. Assume that $\left(B_{i}\right)_{i \in \mathbb{N}}$ is a sequence of open balls such that $\operatorname{dim}_{\mathrm{H}} \mathbf{E}\left(\mathbf{x},\left(B_{i}\right)_{i \in \mathbb{N}}\right)=s$ for $\mathbb{P}$-almost all $\mathbf{x} \in\left(\mathbb{T}^{d}\right)^{\mathbb{N}}$. Let $\left(r_{i}\right)_{i \in \mathbb{N}}$ be a decreasing sequence of positive real numbers which tends to 0 so slowly that $\mathbf{E}\left(\mathbf{x},\left(B\left(0, \frac{r_{i}}{2}\right)_{i \in \mathbb{N}}\right)\right)=\mathbb{T}^{d}$ for $\mathbb{P}$-almost all $\mathbf{x} \in\left(\mathbb{T}^{d}\right)^{\mathbb{N}}$ (for the existence of such $\left(r_{i}\right)_{i \in \mathbb{N}}$, see [36]). Viewing $\mathbb{T}^{d}=\left[-\frac{1}{2}, \frac{1}{2}\left[{ }^{d} \subset \mathbb{R}^{d}\right.\right.$, we define $A_{i}:=r_{i} F \cup B_{i}$ for all $i \in \mathbb{N}$ and set $\mathbf{A}:=\left(A_{i}\right)_{i \in \mathbb{N}}$.

The fact that $\operatorname{dim}_{\mathrm{H}} F<d$ implies that $\mathcal{L}(F)=0$, and hence, $G_{t}\left(A_{i}\right)=G_{t}\left(B_{i}\right)$ for all $i \in \mathbb{N}$, giving $s_{0}(\mathbf{A})=s$ [recall (1.8)]. By [14], Theorem 2, we have $F \in$ $\mathcal{G}^{t}(]-\frac{1}{2}, \frac{1}{2}\left[{ }^{d}\right)$. Let $\widetilde{F}$ be the lift of $F$ to $\mathbb{R}^{d}$ by a covering map. We claim that $\widetilde{F} \in \mathcal{G}^{t}\left(\mathbb{R}^{d}\right)$. Indeed, to prove this claim, by [13], Lemma 10 , it is enough to show that the equality in (7.1) (in which $F$ is replaced by $\widetilde{F}$ ) holds for all dyadic cubes $Q$ with small diameter. This is the case, since $F \in \mathcal{G}^{t}(]-\frac{1}{2}, \frac{1}{2}\left[{ }^{d}\right)$ and $\widetilde{F}$ is the lift of $F$. Since $\mathcal{G}^{t}\left(\mathbb{R}^{d}\right)$ is closed under countable intersections and similarities by [20], Theorem A, we obtain $\widetilde{H}(\mathbf{x}):=\bigcap_{i=n}^{\infty}\left(x_{i}+r_{i} \widetilde{F}\right) \in \mathcal{G}^{t}\left(\mathbb{R}^{d}\right)$ for all $\mathbf{x} \in \mathbb{T}^{d}$, and thus, $H(\mathbf{x}):=\widetilde{H}(\mathbf{x}) \cap]-\frac{1}{2}, \frac{1}{2}\left[{ }^{d} \in \mathcal{G}^{t}(]-\frac{1}{2}, \frac{1}{2}\left[{ }^{d}\right)\right.$ by [13], Proposition 1.

Since $\mathbf{E}\left(\mathbf{x},\left(B\left(0, \frac{r_{i}}{2}\right)_{i \in \mathbb{N}}\right)\right)=\mathbb{T}^{d}$ for $\mathbb{P}$-almost all $\mathbf{x} \in \mathbb{T}^{d}$, every point of $\mathbb{T}^{d}$ belongs to $B\left(x_{i}, \frac{r_{i}}{2}\right)$ for infinitely many $i \in \mathbb{N}$. Using the fact that the sequence $\left(r_{i}\right)_{i \in \mathbb{N}}$ tends to zero, we conclude that $]-\frac{1}{2}, \frac{1}{2}\left[{ }^{d} \subset \bigcup_{i=n}^{\infty} B\left(x_{i}, \frac{r_{i}}{2}\right) \cap\right]-\frac{1}{2}, \frac{1}{2}\left[{ }^{d}\right.$ for all $n \in \mathbb{N}$. Combining this with the fact $\left.\widetilde{H}(\mathbf{x}) \cap B\left(x_{i}, \frac{r_{i}}{2}\right) \cap\right]-\frac{1}{2}, \frac{1}{2}\left[{ }^{d} \subset x_{i}+r_{i} F\right.$ for all $i \geq n$, leads to $H(\mathbf{x}) \subset \bigcup_{i=n}^{\infty}\left(x_{i}+r_{i} F\right)$ for $\mathbb{P}$-almost all $\mathbf{x} \in \mathbb{T}^{d}$. By [13], Proposition 1, every $G_{\delta}$-set containing a subset in $\mathcal{G}^{t}(]-\frac{1}{2}, \frac{1}{2}\left[{ }^{d}\right)$ belongs to $\mathcal{G}^{t}(]-\frac{1}{2}, \frac{1}{2}\left[{ }^{d}\right)$. Thus, $\mathbb{P}$-almost surely, $\operatorname{dim}_{\mathrm{H}}\left(\bigcap_{n=1}^{\infty} \bigcup_{i=n}^{\infty}\left(x_{i}+r_{i} F\right)\right) \geq t$, giving

$$
\operatorname{dim}_{\mathrm{H}} \mathbf{E}(\mathbf{x}, \mathbf{A}) \geq \operatorname{dim}_{\mathrm{H}} \mathbf{E}\left(\mathbf{x},\left(r_{i} F\right)_{i \in \mathbb{N}}\right) \geq t>s=s_{0}(\mathbf{A})
$$

for $\mathbb{P}$-almost all $\mathbf{x} \in\left(\mathbb{T}^{d}\right)^{\mathbb{N}}$.

Finally, we give examples which show that Theorem 1.1 fails if the distribution $\sigma$ is singular with respect to the Lebesgue measure.

EXAMPLE 7.5. (a) Let $f(x, y)$ be as in Example 7.4 and let $\sigma:=\delta_{x_{0}}$ for some $x_{0} \in \mathbb{T}^{d}$. Set $\mathbb{P}:=\prod_{i=1}^{\infty} \sigma$. Defining $A_{n}:=B\left(0, n^{-\frac{1}{d}}\right) \backslash\{0\}$, we obtain $s_{0}(\mathbf{A})=t_{0}(\mathbf{A})=d$. However, $\lim \sup _{n \rightarrow \infty}\left(x_{n}+A_{n}\right)=\varnothing \mathbb{P}$-almost surely. Thus Theorem 1.1 is not valid. 
(b) Let $s<d$ and let $C$ be the regular $2^{d}$-corner Cantor set on $\mathbb{T}^{d}$ with $\operatorname{dim}_{\mathrm{H}} C=$ $\operatorname{dim}_{P} C=s$. Set $\sigma:=\left.\mathcal{H}^{s}\right|_{C}$ and assume that everything else is as in example (a). Then $\mathbf{E}(\mathbf{x}, \mathbf{A}) \subset C$ almost surely. In particular,

$$
\operatorname{dim}_{\mathrm{H}} \mathbf{E}(\mathbf{x}, \mathbf{A}) \leq \operatorname{dim}_{\mathbf{P}} \mathbf{E}(\mathbf{x}, \mathbf{A}) \leq s<s_{0}(\mathbf{A})=t_{0}(\mathbf{A})=d
$$

$\mathbb{P}$-almost surely. Hence, for every $s<d$ there exists a measure $\sigma$ with $\operatorname{dim}_{\mathrm{H}} \sigma=s$ for which Theorem 1.1 fails.

REMARK 7.6. Seuret [49] and Ekström and Persson [17] have recently obtained results for dimensions of random covering sets generated by balls which are distributed according to singular measures. These results give further examples demonstrating that the assumption of nonsingularity of $\sigma$ is necessary for the validity of Theorem 1.1.

\section{Further generalisations and remarks.}

8.1. A weak large intersection property of random covering sets. In [14, 47], it is proved that, when $\mathbf{A}=\left(A_{n}\right)_{n \in \mathbb{N}}$ is a sequence of open balls or general open sets on $\mathbb{T}^{d}$ so that $\sum_{n=1}^{\infty} g_{s}\left(A_{n}\right)=\infty$ for some $0<s \leq d$, then almost surely the random covering set $\mathbf{E}(\mathbf{x}, \mathbf{A})$ has the large intersection property in the sense that $\mathbf{E}(\mathbf{x}, \mathbf{A}) \in \mathcal{G}^{s}$ (cf. Definition 7.3). We remark that this result also holds under a weaker condition that $\sum_{n=1}^{\infty} G_{s}\left(A_{n}\right)=\infty$, because one may find open subsets $B_{n}$ of $A_{n}$ so that $\sum_{n=1}^{\infty} g_{s}\left(B_{n}\right)=\infty$, according to the following easily checked fact:

$$
G_{S}(A)=\sup \left\{g_{s}(B): B \subset A, B \text { is open }\right\}
$$

whenever $A$ is open. We emphasise that, in the above investigation, the assumption of $A_{n}$ being open is essential and cannot be dropped, for otherwise $\mathbf{E}(\mathbf{x}, \mathbf{A})$ may not be a $G_{\delta}$-set.

Nevertheless, in the general setting that the sets in $\mathbf{A}$ are Lebesgue measurable, we obtain the following weak large intersection property of random covering sets.

THEOREM 8.1. Assuming that $\mathbf{A}$ is a sequence of Lebesgue measurable sets, we have under the conditions of Theorem 1.1 that

$$
\operatorname{dim}_{H}\left(\bigcap_{j=1}^{\infty} \mathbf{E}\left(\mathbf{x}^{j}, \mathbf{A}\right)\right) \geq s_{0}(\mathbf{A})
$$

for $\left(\prod_{j=1}^{\infty} \mathbb{P}\right)$-almost all $\left(\mathbf{x}^{j}\right)_{j \in \mathbb{N}} \in \prod_{j=1}^{\infty} U^{\mathbb{N}}$.

PROOF. This can be verified by modifying the proof of Proposition 4.5 in the following manner: Let $\varphi: \mathbb{N} \rightarrow \mathbb{N} \times \mathbb{N}$ be a bijection obtained using the diagonal method. Repeat the construction of Proposition 4.5 such that the $n$th construction 
step is done using the variable $\mathbf{x}^{\varphi(n)_{1}}$, where $\varphi(n)_{1}$ is the first coordinate of $\varphi(n)$. This leads to the conclusion

$$
\operatorname{dim}_{\mathrm{H}}\left(\bigcap_{n=1}^{\infty} \bigcup_{i=N_{n-1}+1}^{N_{n}} A_{i}\left(x_{i}^{\varphi(n)_{1}}\right)\right) \geq s
$$

[cf. (4.9)], which implies the desired result.

8.2. Hausdorff measure of random covering sets. Let $d \in \mathbb{N}$. Denote by $\mathfrak{G}$ the collection of functions $h:[0, \infty[\rightarrow[0, \infty$ [ such that $h$ is increasing, positive near $0, \lim _{r \rightarrow 0} h(r)=h(0)=0$ and $h(r) r^{-d}$ is decreasing. Any element of $\mathfrak{G}$ is called a gauge function. For $F \subset \mathbb{R}^{d}$ and $h \in \mathfrak{G}$, we use $\mathcal{H}^{h}(F)$ and $\mathcal{H}_{\infty}^{h}(F)$ to denote the Hausdorff measure and Hausdorff content of $F$ with respect to the gauge function $h$ (cf. $[10,48]$ ). For instance, $\mathcal{H}_{\infty}^{h}(F)$ is defined by replacing $\left(\operatorname{diam} F_{n}\right)^{s}$ by $h\left(\operatorname{diam} F_{n}\right)$ in the definition (1.6).

In [14], Durand studied the Hausdorff measures of random covering sets on $\mathbb{T}^{d}$ when $\mathbf{A}$ is a sequence of balls of the form $A_{n}=B\left(0, r_{n}\right)$. Using the mass transference principle established in [3], he showed that, for any $h \in \mathfrak{G}$ with $\lim _{r \rightarrow 0} h(r) r^{-d}=\infty$, almost surely

$$
\mathcal{H}^{h}(\mathbf{E}(\mathbf{x}, \mathbf{A}))= \begin{cases}\infty & \text { if } \sum_{n=1}^{\infty} h\left(r_{n}\right)=\infty \\ 0 & \text { otherwise }\end{cases}
$$

However, this approach does not extend to the general case when the sets in $\mathbf{A}$ are not ball-like, since the mass transference principle may fail in such situation.

To deal with the general case, let us introduce some notation. For a Lebesgue measurable set $F \subset \mathbb{R}^{d}$ with $\mathcal{L}(F)>0$ and $h \in \mathfrak{G}$, we define the $h$-energy of $F$ by

$$
I_{h}(F):=\iint_{F \times F} h(|x-y|)^{-1} d \mathcal{L}(x) d \mathcal{L}(y) .
$$

Set $g_{h}(F):=\mathcal{L}(F)^{2} I_{h}(F)^{-1}$ and use $g_{h}$ to define $G_{h}(F)$ as in (1.9). Following the argument in the proof of Lemma 3.2 with routine changes, we can show that

$$
\mathcal{H}_{\infty}^{h}(F) \geq G_{h}(F)
$$

As a generalisation of Theorem 1.1, we have the following result on the Hausdorff measures of general random covering sets.

THEOREM 8.2. Let $h \in \mathfrak{G}$. Under the assumptions of Theorem 1.1, we have:

(i) $\sum_{n=1}^{\infty} \mathcal{H}_{\infty}^{h}\left(A_{n}\right)<\infty \Longrightarrow \mathcal{H}^{h}(\mathbf{E}(\mathbf{x}, \mathbf{A}))=0$.

(ii) $\sum_{n=1}^{\infty} G_{h}\left(A_{n}\right)=\infty \Longrightarrow \mathcal{H}^{h}(\mathbf{E}(\mathbf{x}, \mathbf{A}))=\infty$ for $\mathbb{P}$-almost all $\mathbf{x} \in U^{\mathbb{N}}$, provided that $I_{h}(B(0, R))<\infty$ for all $R>0$ and $A_{n}$ are Lebesgue measurable. 
(iii) Assume that $r \mapsto h(r) r^{-d+\varepsilon}$ is decreasing for some $\varepsilon>0$ and, moreover, assume that $\tilde{h} \in \mathfrak{G}$ is such that the inequality $\tilde{h}(r) \leq h(r)^{1+\delta}$ is valid for some $\delta>0$ and all $r>0$. Then

$$
\sum_{n=1}^{\infty} G_{h}\left(A_{n}\right)<\infty \quad \Longrightarrow \quad \sum_{n=1}^{\infty} \mathcal{H}_{\infty}^{\tilde{h}}\left(A_{n}\right)<\infty
$$

provided that $A_{n}$ are Lebesgue measurable with positive Lebesgue density.

PROOF. Statement (i) follows from a routine modification of the proof of Lemma 3.1. Statement (ii) follows from the proof of Theorem 1.1(b) with slight modifications. Indeed, in the proof of Theorem 1.1(b), the only place where the fact that the kernel is $|x|^{-s}$ is needed is inequality (2.11) (see the proof of Lemma 2.4). To extend that inequality associated to $h$, it is enough to have that

$$
h(r) \leq(1+O(\varepsilon)) h((1-\varepsilon) r) \quad \text { for all } 0<r<2 R .
$$

Note that $h$ is doubling in the sense that $h(2 r)<c h(r)$ for some constant $c>1$, which follows from the fact that $h(r) r^{-d}$ is decreasing. Hence, the gauge function $\tilde{h}$ obtained from $h$ as the linear interpolation of $h$ at points $2^{-n}, n \in \mathbb{N}$, is equivalent with $h$ and satisfies (8.2). Now Proposition 4.5 implies that $\mathcal{H}^{h}(\mathbf{E}(\mathbf{x}, \mathbf{A}))>0$ $\mathbb{P}$-almost surely. It is not difficult to see that if $\sum_{n=1}^{\infty} G_{h}\left(A_{n}\right)=\infty$ there exists a gauge function $h^{\prime}$ such that $\lim _{r \rightarrow 0} h^{\prime}(r) h(r)^{-1}=0$ and $\sum_{n=1}^{\infty} G_{h^{\prime}}\left(A_{n}\right)=\infty$. Therefore, $\mathcal{H}^{h^{\prime}}(\mathbf{E}(\mathbf{x}, \mathbf{A}))>0$ which implies $\mathcal{H}^{h}(\mathbf{E}(\mathbf{x}, \mathbf{A}))=\infty$.

The proof of (iii) is essentially identical to that of Lemma 3.10. Observe that one may assume that $\mathcal{H}^{\tilde{h}}(B(0, R))>0$ for some $R>0$ since otherwise the claim is trivial. The assumption that $h(r) r^{-d+\varepsilon}$ is decreasing is needed at the end of the proof of Lemma 3.7 when the term $(I I)$ is estimated. (Recall that Lemma 3.7 is needed in the proof of Proposition 3.8.) Observe that heuristically $\mathcal{H}^{\tilde{h}}(B(0, R))>$ 0 means that $\tilde{h}(r)$ should be larger than $r^{d}$ for small $r>0$ and, therefore, $h(r)$ should be larger than $r^{\frac{d}{1+\delta}}$ for small $r>0$.

REMARK 8.3. One may expect that, for some $R>0$, there exists a constant $C>0$ such that, for all Lebesgue measurable sets $F \subset B(0, R)$,

$$
\mathcal{H}_{\infty}^{h}(F) \leq C G_{h}(F) .
$$

If so, the condition $\sum_{n=1}^{\infty} G_{h}\left(A_{n}\right)=\infty$ in Theorem 8.2(ii) can be replaced by

$$
\sum_{n=1}^{\infty} \mathcal{H}_{\infty}^{h}\left(A_{n}\right)=\infty
$$

However, (8.3) does not hold for general doubling gauge functions even in the case where $F$ is a ball. Indeed, let $h(r)=r^{d}(\log r)^{2}$ for all $0<r<r_{0}$, where $r_{0}$ is chosen such that $h$ is increasing. A straightforward calculation implies that $I_{h}(B(x, r))$ 
is comparable to $\left(r^{d}|\log r|\right)^{-1}$. Applying [45], Theorem 1.15, to product measures, making a discrete approximation and using the fact that the sum $\sum_{i=1}^{n} a_{i}^{2}$ is minimised for the uniform probability vector $\left(a_{1}, \ldots, a_{n}\right)$, it is not difficult to see that $g_{h}(B(x, r))$ is comparable to $G_{h}(B(x, r))$. Therefore, $G_{h}(B(x, r))$ is comparable to $h(r)|\log r|^{-1}$ while $\mathcal{H}_{\infty}^{h}(B(x, r))$ is comparable to $h(r)$.

REMARK 8.4. Here, we indicate how $G_{h}(F)$ can be calculated for some concrete examples. Assume that $F=B(x, r)$. It follows immediately from the definition that $G_{h}(F) \leq h(2 r)$. If $h(r) r^{-d+\varepsilon}$ is decreasing for some $\varepsilon>0$ (thus $h$ is doubling), one easily sees that $I_{h}(F) \leq C r^{2 d} h(r)^{-1}$ for some constant $C>0$. Therefore, $G_{h}(F)$ is comparable to $h(r)$. Another easily calculable example is when $F$ is a rectangle (or parallelepiped in higher dimensions) with side lengths $a \geq b$. Then $G_{s}(F)$ is comparable to $a^{s}$ for $0<s<1$ and to $a b^{s-1}$ for $1<s<2$.

REMARK 8.5. Basing on the above remark, one can verify that (8.3) holds in the following particular cases: (i) $F$ is a ball and $h$ is a gauge function so that $r \mapsto h(r) r^{-d+\varepsilon}$ is decreasing for some $\varepsilon>0$; (ii) $F$ is a rectangle, and $h(r)=r^{s}$ for some noninteger $s \in(0,2)$.

8.3. A question on the measurability of level sets of random covering sets. It is a natural question whether $\operatorname{dim} \mathbf{E}(\mathbf{x}, \mathbf{A})$ takes a constant value almost surely in the general setting that $\mathbf{A}$ is a sequence of Lebesgue measurable sets, where dim is either the Hausdorff, packing or box counting dimension. It is obvious that $\operatorname{dim} \mathbf{E}(\mathbf{x}, \mathbf{A})$ does not depend on a finite number of coordinates $x_{i}$. Therefore,

$$
F_{s}:=\left\{\mathbf{x} \in U^{\mathbb{N}}: \operatorname{dim} \mathbf{E}(\mathbf{x}, \mathbf{A})=s\right\}
$$

is a tail event for every $0 \leq s \leq d$, provided that $F_{s}$ is measurable. In this case, the Kolmogorov's zero-one law would imply that $\mathbf{x} \mapsto \operatorname{dim} \mathbf{E}(\mathbf{x}, \mathbf{A})$ is almost surely a constant. Theorem 1.1 gives the value of this constant under further assumptions on $\mathbf{A}$.

Using the results of Dellacherie [12] and Mattila and Mauldin [46], it is easy to see that $F_{s}$ is measurable with respect to the $\sigma$-algebra generated by analytic sets provided that the sets $A_{n}$ are analytic for all $n \in \mathbb{N}$ (for details see [32]). For Lebesgue measurable generating sets $\left(A_{n}\right)_{n \in \mathbb{N}}$, we do not know whether the sets $F_{s}$ are measurable or not.

Acknowledgements. We thank Henna Koivusalo and Antti Käenmäki for interesting discussions and the referees for useful comments.

\section{REFERENCES}

[1] BARRAL, J. and FAN, A.-H. (2005). Covering numbers of different points in Dvoretzky covering. Bull. Sci. Math. 129 275-317. MR2134123 
[2] Beresnevich, V., Dickinson, D. and Velani, S. (2006). Measure theoretic laws for lim sup sets. Mem. Amer. Math. Soc. 179 x+91. MR2184760

[3] Beresnevich, V. and Velani, S. (2006). A mass transference principle and the Duffin-Schaeffer conjecture for Hausdorff measures. Ann. of Math. (2) 164 971-992. MR2259250

[4] BeRniK, V. I. and Dodson, M. M. (1999). Metric Diophantine Approximation on Manifolds. Cambridge Tracts in Mathematics 137. Cambridge Univ. Press, Cambridge. MR1727177

[5] Besicovitch, A. S. (1935). On the sum of digits of real numbers represented in the dyadic system. Math. Ann. 110 321-330. MR1512941

[6] Billard, P. (1965). Séries de Fourier aléatoirement bornées, continues, uniformément convergentes. Ann. Sci. École Norm. Sup. (3) 82 131-179. MR0182832

[7] Borel, E. (1897). Sur les séries de Taylor. Acta Math. 21 243-247. MR1554891

[8] Bugeaud, Y. (2003). A note on inhomogeneous Diophantine approximation. Glasg. Math. J. 45 105-110. MR1972699

[9] Bugeaud, Y. (2004). Intersective sets and Diophantine approximation. Michigan Math. J. 52 667-682. MR2097404

[10] Carleson, L. (1967). Selected Problems on Exceptional Sets. Van Nostrand Mathematical Studies 13. Van Nostrand, Princeton, NJ. MR0225986

[11] Chen, C., Koivusalo, H., Li, B. and Suomala, V. (2014). Projections of random covering sets. J. Fractal Geom. 1 449-467. MR3299820

[12] Dellacherie, C. (1972). Ensembles Analytiques, Capacités, Mesures de Hausdorff. Lecture Notes in Mathematics 295. Springer, Berlin. MR0492152

[13] Durand, A. (2008). Sets with large intersection and ubiquity. Math. Proc. Cambridge Philos. Soc. 144 119-144. MR2388238

[14] DuRAnd, A. (2010). On randomly placed arcs on the circle. In Recent Developments in Fractals and Related Fields. Appl. Numer. Harmon. Anal. 343-351. Birkhäuser, Boston, MA. MR2743004

[15] Dvoretzky, A. (1956). On covering a circle by randomly placed arcs. Proc. Natl. Acad. Sci. USA 42 199-203. MR0079365

[16] Eggleston, H. G. (1949). The fractional dimension of a set defined by decimal properties. Quart. J. Math., Oxford Ser. 20 31-36. MR0031026

[17] Ekström, F. and Persson, T. (2016). Hausdorff dimension of random limsup sets. Preprint. Available at arXiv:1612.07110 [math.CA].

[18] El HÉlou, Y. (1978). Recouvrement du tore $T^{q}$ par des ouverts aléatoires et dimension de Hausdorff de l'ensemble non recouvert. C. R. Acad. Sci. Paris Sér. A-B 287 A815-A818. MR0538501

[19] ERdôs, P. (1961). Some unsolved problems. Magyar Tud. Akad. Mat. Kutató Int. Közl. 6 221254. MR0177846

[20] FAlconer, K. J. (1994). Sets with large intersection properties. J. Lond. Math. Soc. (2) 49 267-280. MR1260112

[21] FAlCONER, K. J. (2003). Fractal Geometry: Mathematical Foundations and Applications, 2nd ed. Wiley, Hoboken, NJ. MR2118797

[22] FAN, A. (2002). How many intervals cover a point in Dvoretzky covering? Israel J. Math. 131 157-184. MR1942307

[23] FAn, A. H. and Kahane, J.-P. (1993). Rareté des intervalles recouvrant un point dans un recouvrement aléatoire. Ann. Inst. Henri Poincaré Probab. Stat. 29 453-466. MR1246642

[24] FAn, A.-H., Schmeling, J. and Troubetzkoy, S. (2013). A multifractal mass transference principle for Gibbs measures with applications to dynamical Diophantine approximation. Proc. Lond. Math. Soc. (3) 107 1173-1219. MR3126394

[25] FAN, A.-H. and WU, J. (2004). On the covering by small random intervals. Ann. Inst. Henri Poincaré Probab. Stat. 40 125-131. MR2037476 
[26] Harman, G. (1998). Metric Number Theory. London Mathematical Society Monographs. New Series 18. Clarendon Press, New York. MR1672558

[27] Hawkes, J. (1973). On the covering of small sets by random intervals. Quart. J. Math. Oxford Ser. (2) 24 427-432. MR0324748

[28] Hill, R. and Velani, S. L. (1999). The shrinking target problem for matrix transformations of tori. J. Lond. Math. Soc. (2) 60 381-398. MR1724857

[29] HofFMAnN-Jørgensen, J. (1973). Coverings of metric spaces with randomly placed balls. Math. Scand. 32 169-186. MR0341556

[30] Janson, S. (1986). Random coverings in several dimensions. Acta Math. 156 83-118. MR0822331

[31] JÄrvenpäÄ, E., JÄrvenpÄÄ, M., Koivusalo, H., Li, B. and SuOMAla, V. (2014). Hausdorff dimension of affine random covering sets in torus. Ann. Inst. Henri Poincaré Probab. Stat. 50 1371-1384. MR3269998

[32] JärvenpäÄ, E., JärvenpäÄ, M., Koivusalo, H., Li, B., Suomala, V. and Xiao, Y. (2017). Hitting probabilities of random covering sets in tori and metric spaces. Electron. J. Probab. 22 Paper No. 1, 18. MR3613694

[33] Jonasson, J. and Steif, J. E. (2008). Dynamical models for circle covering: Brownian motion and Poisson updating. Ann. Probab. 36 739-764. MR2393996

[34] Kahane, J.-P. (1959). Sur le recouvrement d'un cercle par des arcs disposés au hasard. C. $R$. Math. Acad. Sci. Paris 248 184-186. MR0103533

[35] Kahane, J.-P. (1985). Some Random Series of Functions, 2nd ed. Cambridge Studies in Advanced Mathematics 5. Cambridge Univ. Press, Cambridge. MR0833073

[36] Kahane, J.-P. (1990). Recouvrements aléatoires et théorie du potentiel. Colloq. Math. 60/61 387-411. MR1096386

[37] Kahane, J.-P. (2000). Random coverings and multiplicative processes. In Fractal Geometry and Stochastics, II (Greifswald/Koserow, 1998). Progress in Probability 46 125-146. Birkhäuser, Basel. MR1785624

[38] KeCHRIS, A. S. (1995). Classical Descriptive Set Theory. Graduate Texts in Mathematics 156. Springer, New York. MR1321597

[39] Khoshnevisan, D., Peres, Y. and XiaO, Y. (2000). Limsup random fractals. Electron. J. Probab. 5 no. 5, 24. MR1743726

[40] Landkof, N. S. (1972). Foundations of Modern Potential Theory. Springer, New York. MR0350027

[41] Li, B., SHIEH, N.-R. and XIAO, Y. (2013). Hitting probabilities of the random covering sets. In Fractal Geometry and Dynamical Systems in Pure and Applied Mathematics. II. Fractals in Applied Mathematics. Contemp. Math. 601 307-323. Amer. Math. Soc., Providence, RI. MR3203868

[42] Liao, L. and SEuRET, S. (2013). Diophantine approximation by orbits of expanding Markov maps. Ergodic Theory Dynam. Systems 33 585-608. MR3035299

[43] Mandelbrot, B. B. (1972). Renewal sets and random cutouts. Z. Wahrsch. Verw. Gebiete 22 145-157. MR0309162

[44] Mandelbrot, B. B. (1972). On Dvoretzky coverings for the circle. Z. Wahrsch. Verw. Gebiete 22 158-160. MR0309163

[45] Mattila, P. (1995). Geometry of Sets and Measures in Euclidean Spaces: Fractals and Rectifiability. Cambridge Studies in Advanced Mathematics 44. Cambridge Univ. Press, Cambridge. MR1333890

[46] Mattila, P. and Mauldin, R. D. (1997). Measure and dimension functions: Measurability and densities. Math. Proc. Cambridge Philos. Soc. 121 81-100. MR1418362

[47] Persson, T. (2015). A note on random coverings of tori. Bull. Lond. Math. Soc. 47 7-12. MR3312958 
[48] Rogers, C. A. (1970). Hausdorff Measures. Cambridge Univ. Press, London. MR0281862

[49] SEURET, S. (2017). Inhomogeneous coverings of topological Markov shifts. Math. Proc. Cambridge Philos. Soc. To appear. Available at https://doi.org/10.1017/S0305004117000512.

[50] ShePp, L. A. (1972). Covering the circle with random arcs. Israel J. Math. 11 328-345. MR0295402

[51] SHEPP, L. A. (1972). Covering the line with random intervals. Z. Wahrsch. Verw. Gebiete 23 163-170. MR0322923

\section{D.-J. FENG}

DEPARTMENT OF MATHEMATICS

LADY SHAW BUILDING

CHINESE UNIVERSiTy OF HONG KONG

Shatin, N. T.

HONG KONG

E-MAIL: djfeng@math.cuhk.edu.hk

\author{
E. JÄRVENPÄÄ \\ M. JÄRVENPÄÄ \\ V. SUOMALA \\ Department of Mathematical Sciences \\ UNIVERSITY OF OULU \\ P.O. BOX 3000 \\ 90014 FINLAND \\ E-MAIL: esa.jarvenpaa@oulu.fi \\ maarit.jarvenpaa@oulu.fi \\ ville.suomala@oulu.fi
}

